\title{
THE THIRD CATALOG OF ACTIVE GALACTIC NUCLEI DETECTED BY THE FERMI LARGE AREA TELESCOPE
}

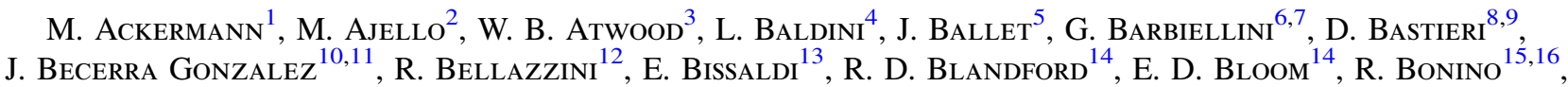
E. Bottacini ${ }^{14}$, T. J. Brandt ${ }^{10}$, J. Bregeon ${ }^{17}$, R. J. Britto ${ }^{18}$, P. Bruel $^{19}$, R. Buehler ${ }^{1}$, S. Buson ${ }^{8,9}$, G. A. Caliandro ${ }^{14,20}$,

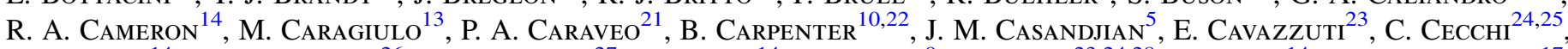
E. Charles $^{14}$, A. CheKhtman ${ }^{26}$, C. C. Cheung ${ }^{27}$, J. Chiang ${ }^{14}$, G. Chiaro ${ }^{9}$, S. Ciprini ${ }^{23,24,28}$, R. Claus ${ }^{14}$, J. Cohen-Tanugi ${ }^{17}$, L. R. Cominsky ${ }^{29}$, J. Conrad ${ }^{30,31,32,70}$, S. Cutini ${ }^{23,24,28}$, R. D’Abrusco 33 , F. D’Ammando ${ }^{34,35}$, A. DE Angelis ${ }^{36}$,

R. Desiante ${ }^{6,37}$, S. W. Digel ${ }^{14}$, L. Di Venere ${ }^{38}$, P. S. Drell ${ }^{14}$, C. Favuzzi $^{13,38}{ }^{14}$, S. J. Fegan ${ }^{19}$, E. C. Ferrara ${ }^{10}$, J. FinKe $^{27}$, W. B. Focke ${ }^{14}$, A. Franckowiak ${ }^{14}$, L. Fuhrmann ${ }^{39}$, Y. Fukazawa ${ }^{40}$, A. K. Furniss ${ }^{14}$, P. Fusco ${ }^{13,38}$, F. Gargano ${ }^{13}$, D. Gasparrini ${ }^{23,24,28}$, N. Giglietto ${ }^{13,38}$, P. Giommi ${ }^{23}$, F. Giordano $^{13,38}$, M. Giroletti ${ }^{34}$, T. Glanzman ${ }^{14}$, G. Godfrey ${ }^{14}$,

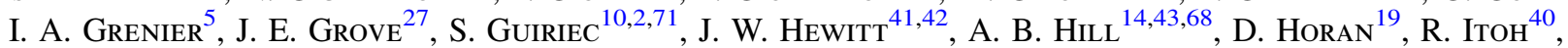
G. Jóhannesson ${ }^{44}$, A. S. Johnson ${ }^{14}$, W. N. Johnson ${ }^{27}$, J. KataOKa ${ }^{45}$, T. Kawano ${ }^{40}$, F. Krauss ${ }^{46}$, M. Kuss ${ }^{12}$, G. La Mura ${ }^{9,47}$,

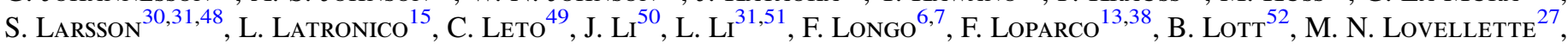
P. Lubrano $^{24,25}$, G. M. Madejski ${ }^{14}$, M. Mayer ${ }^{1}$, M. N. Mazziotta ${ }^{13}$, J. E. McEnery ${ }^{10,11}$, P. F. Michelson ${ }^{14}$, T. Mizuno $^{53}$, A. A. Moiseev ${ }^{11,42}$, M. E. MonZani ${ }^{14}$, A. Morsellit ${ }^{54}$, I. V. Moskalenko ${ }^{14}$, S. Murgia ${ }^{55}$, E. Nuss ${ }^{17}$, M. Ohno ${ }^{40}$, T. Ohsugi ${ }^{53}$, R. Ojha ${ }^{10}$, N. Omodei ${ }^{14}$, M. Orienti ${ }^{34}$, E. Orlando ${ }^{14}$, A. Paggi ${ }^{33}$, D. Paneque ${ }^{14,56}$, J. S. Perkins ${ }^{10}$, M. Pesce-Rollins ${ }^{12}$,

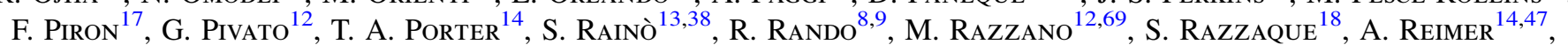
O. Reimer ${ }^{14,47}$, R. W. Romani ${ }^{14}$, D. SAlvetti ${ }^{21}$, M. SchaAl ${ }^{57}$, F. K. Schinzel ${ }^{58}$, A. Schulz ${ }^{1}$, C. Sgrò ${ }^{12}$, E. J. Siskind ${ }^{59}$, K. V. Sokolovsky ${ }^{39,60}$, F. Spada ${ }^{12}$, G. Spandre ${ }^{12}$, P. Spinelli ${ }^{13,38}$, L. StaWarz ${ }^{61,62}$, D. J. Suson ${ }^{63}$, H. Takahashi ${ }^{40}$,

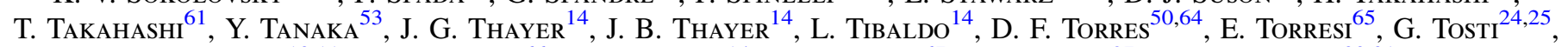
E. Troja ${ }^{10,11}$, Y. Uchiyama ${ }^{66}$, G. Vianello ${ }^{14}$, B. L. Winer ${ }^{67}$, K. S. Wood ${ }^{27}$, and S. Zimmer ${ }^{30,31}$

${ }^{1}$ Deutsches Elektronen Synchrotron DESY, D-15738 Zeuthen, Germany

2 Department of Physics and Astronomy, Clemson University, Kinard Lab of Physics, Clemson, SC 29634-0978, USA

${ }^{3}$ Santa Cruz Institute for Particle Physics, Department of Physics and Department of Astronomy and Astrophysics, University of California at Santa Cruz, Santa Cruz, CA 95064, USA

${ }^{4}$ Università di Pisa and Istituto Nazionale di Fisica Nucleare, Sezione di Pisa I-56127 Pisa, Italy

${ }^{5}$ Laboratoire AIM, CEA-IRFU/CNRS/Université Paris Diderot, Service d'Astrophysique, CEA Saclay, F-91191 Gif sur Yvette, France

${ }^{6}$ Istituto Nazionale di Fisica Nucleare, Sezione di Trieste, I-34127 Trieste, Italy

${ }^{7}$ Dipartimento di Fisica, Università di Trieste, I-34127 Trieste, Italy

${ }^{8}$ Istituto Nazionale di Fisica Nucleare, Sezione di Padova, I-35131 Padova, Italy

9 Dipartimento di Fisica e Astronomia "G. Galilei," Università di Padova, I-35131 Padova, Italy ${ }^{10}$ NASA Goddard Space Flight Center, Greenbelt, MD 20771, USA

${ }^{11}$ Department of Physics and Department of Astronomy, University of Maryland, College Park, MD 20742, USA

${ }_{12}$ Istituto Nazionale di Fisica Nucleare, Sezione di Pisa, I-56127 Pisa, Italy

${ }^{13}$ Istituto Nazionale di Fisica Nucleare, Sezione di Bari, I-70126 Bari, Italy

${ }^{14}$ W. W. Hansen Experimental Physics Laboratory, Kavli Institute for Particle Astrophysics and Cosmology, Department of Physics and

SLAC National Accelerator Laboratory, Stanford University, Stanford, CA 94305, USA

${ }^{15}$ Istituto Nazionale di Fisica Nucleare, Sezione di Torino, I-10125 Torino, Italy

${ }^{16}$ Dipartimento di Fisica Generale "Amadeo Avogadro," Università degli Studi di Torino, I-10125 Torino, Italy

${ }^{17}$ Laboratoire Univers et Particules de Montpellier, Université Montpellier, CNRS/IN2P3, Montpellier, France

${ }^{18}$ Department of Physics, University of Johannesburg, P. O. Box 524, Auckland Park 2006, South Africa

${ }^{19}$ Laboratoire Leprince-Ringuet, École polytechnique, CNRS/IN2P3, Palaiseau, France

${ }^{20}$ Consorzio Interuniversitario per la Fisica Spaziale (CIFS), I-10133 Torino, Italy

${ }^{21}$ INAF-Istituto di Astrofisica Spaziale e Fisica Cosmica, I-20133 Milano, Italy

${ }^{22}$ Catholic University of America, Washington, DC 20064, USA

${ }^{23}$ Agenzia Spaziale Italiana (ASI) Science Data Center, I-00133 Roma, Italy; elisabetta.cavazzuti@asdc.asi.it, stefano.ciprini@asdc.asi.it, sara.cutini@ asdc.asi.it, gasparrini@ asdc.asi.it

${ }^{24}$ Istituto Nazionale di Fisica Nucleare, Sezione di Perugia, I-06123 Perugia, Italy

${ }^{25}$ Dipartimento di Fisica, Università degli Studi di Perugia, I-06123 Perugia, Italy

${ }^{26}$ College of Science, George Mason University, Fairfax, VA 22030, Resident at Naval Research Laboratory, Washington, DC 20375, USA

${ }_{28}$ Space Science Division, Naval Research Laboratory, Washington, DC 20375-5352, USA

${ }^{28}$ INAF Osservatorio Astronomico di Roma, I-00040 Monte Porzio Catone (Roma), Italy

${ }^{29}$ Department of Physics and Astronomy, Sonoma State University, Rohnert Park, CA 94928-3609, USA

${ }^{30}$ Department of Physics, Stockholm University, AlbaNova, SE-106 91 Stockholm, Sweden

31 The Oskar Klein Centre for Cosmoparticle Physics, AlbaNova, SE-106 91 Stockholm, Sweden

32 The Royal Swedish Academy of Sciences, Box 50005, SE-104 05 Stockholm, Sweden

${ }^{33}$ Harvard-Smithsonian Center for Astrophysics, Cambridge, MA 02138, USA

${ }^{34}$ INAF Istituto di Radioastronomia, I-40129 Bologna, Italy

${ }^{35}$ Dipartimento di Astronomia, Università di Bologna, I-40127 Bologna, Italy

${ }^{36}$ Dipartimento di Fisica, Università di Udine and Istituto Nazionale di Fisica Nucleare, Sezione di Trieste, Gruppo Collegato di Udine, I-33100 Udine, Italy

${ }^{37}$ Università di Udine, I-33100 Udine, Italy

38 Dipartimento di Fisica "M. Merlin” dell’Università e del Politecnico di Bari, I-70126 Bari, Italy

Max-Planck-Institut für Radioastronomie, Auf dem Hügel 69, D-53121 Bonn, Germany

${ }^{40}$ Department of Physical Sciences, Hiroshima University, Higashi-Hiroshima, Hiroshima 739-8526, Japan

${ }^{41}$ Department of Physics and Center for Space Sciences and Technology, University of Maryland Baltimore County, Baltimore, MD 21250, USA 


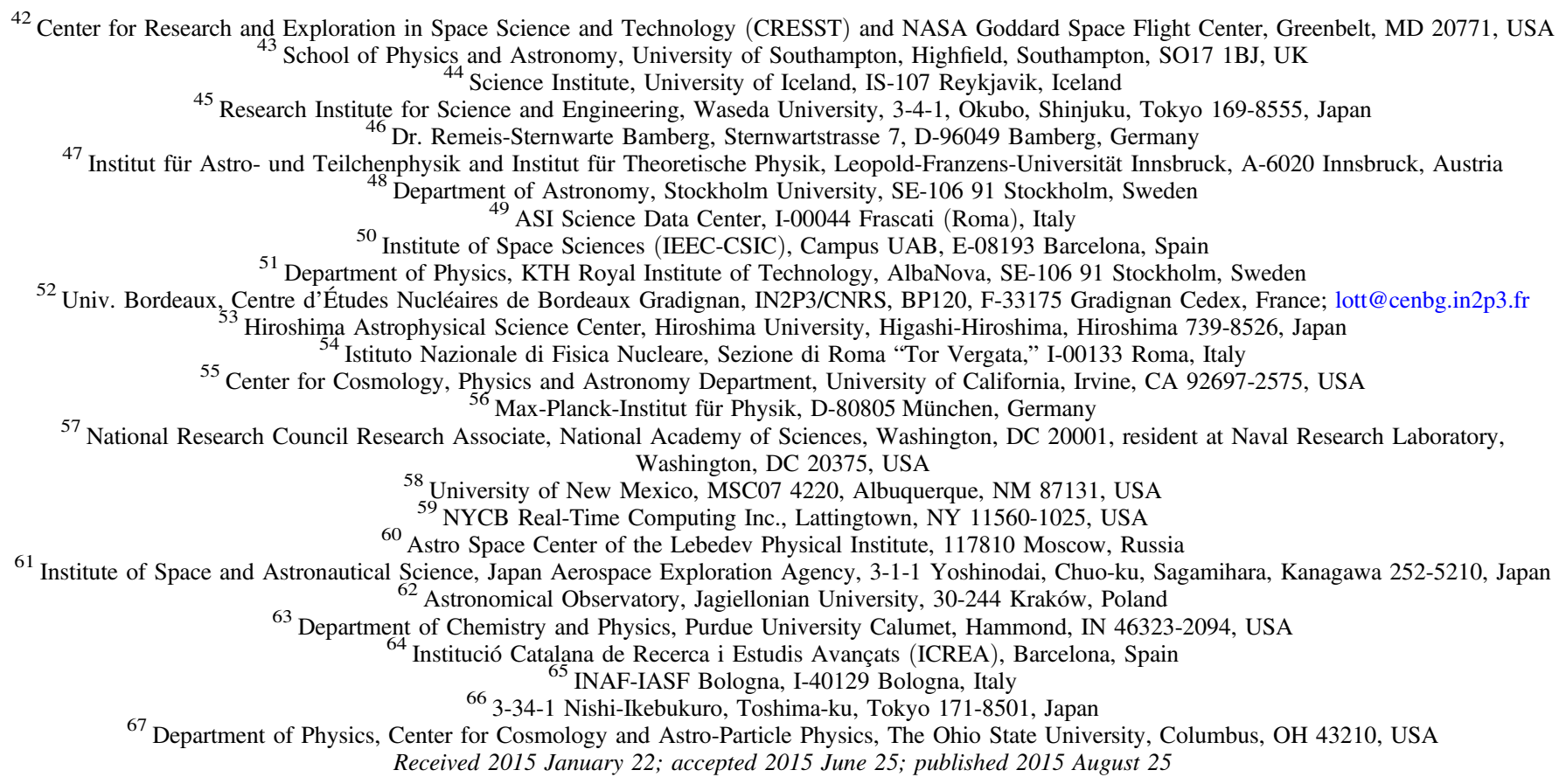

\section{ABSTRACT}

The third catalog of active galactic nuclei (AGNs) detected by the Fermi-LAT (3LAC) is presented. It is based on the third Fermi-LAT catalog (3FGL) of sources detected between $100 \mathrm{MeV}$ and $300 \mathrm{GeV}$ with a Test Statistic greater than 25, between 2008 August 4 and 2012 July 31. The 3LAC includes 1591 AGNs located at high Galactic latitudes $\left(|b|>10^{\circ}\right)$, a $71 \%$ increase over the second catalog based on 2 years of data. There are 28 duplicate associations, thus 1563 of the 2192 high-latitude gamma-ray sources of the 3FGL catalog are AGNs. Most of them (98\%) are blazars. About half of the newly detected blazars are of unknown type, i.e., they lack spectroscopic information of sufficient quality to determine the strength of their emission lines. Based on their gamma-ray spectral properties, these sources are evenly split between flat-spectrum radio quasars (FSRQs) and BL Lacs. The most abundant detected BL Lacs are of the high-synchrotron-peaked (HSP) type. About 50\% of the BL Lacs have no measured redshifts. A few new rare outliers (HSP-FSRQs and high-luminosity HSP BL Lacs) are reported. The general properties of the 3LAC sample confirm previous findings from earlier catalogs. The fraction of 3LAC blazars in the total population of blazars listed in BZCAT remains non-negligible even at the faint ends of the BZCAT-blazar radio, optical, and X-ray flux distributions, which hints that even the faintest known blazars could eventually shine in gamma-rays at LAT-detection levels. The energy-flux distributions of the different blazar populations are in good agreement with extrapolation from earlier catalogs.

Key words: BL Lacertae objects: general - galaxies: active - galaxies: jets - gamma rays: galaxies

Supporting material: machine-readable tables

\section{INTRODUCTION}

Since its launch in 2008, the Fermi-LAT has revolutionized our knowledge of the gamma-ray sky above $100 \mathrm{MeV}$. Its unique combination of high sensitivity, wide field of view, large energy range, and a nominal sky-survey operating mode has enabled a complete mapping and continuous monitoring of the gamma-ray sky to an unprecedented level. Several catalogs or source lists, both general and specialized (active galactic nuclei; AGNs, pulsars, supernova remnants, pulsar wind nebulae, gamma-ray bursts, very-high-energy (VHE)

\footnotetext{
${ }^{68}$ Funded by a Marie Curie IOF, FP7/2007-2013-Grant agreement no. 275861.

69 Funded by contract FIRB-2012-RBFR12PM1F from the Italian Ministry of Education, University and Research (MIUR).

${ }^{70}$ Royal Swedish Academy of Sciences Research Fellow, funded by a grant from the K. A. Wallenberg Foundation.

${ }^{71}$ NASA Postdoctoral Program Fellow, USA.
}

candidates) have already been produced. These constitute important resources to the astronomical community. The successive AGN lists and catalogs, LAT Bright AGN Sample (LBAS; Abdo et al. 2009a), 1LAC (Abdo et al. 2010g) and 2LAC (Ackermann et al. 2011c, 2015), first and second LAT AGN catalogs, respectively, have triggered numerous population studies (e.g., Ghisellini et al. 2009, 2012, 2013; Ajello et al. 2012; D'Abrusco et al. 2012; Massaro et al. 2012; Meyer et al. 2012; Padovani et al. 2012; Finke 2013; Giommi et al. 2013), provided suitable samples, e.g., to probe the Extragalactic Background Light (EBL, Abdo et al. 2010c; Ackermann et al. 2012c), offered suitable target lists to investigate the dichotomy between gamma-ray loud and gamma-ray quiet blazars at other wavelengths (Kovalev et al. 2009; Lister et al. 2009, 2011; Ojha et al. 2010; Giommi et al. 2012; Piner et al. 2012), and served as references for works on individual sources (e.g., Abramowski et al. 2013; Tavecchio et al. 2013). 
This paper presents the third catalog of AGNs detected by the Fermi-LAT after four years of operation (3LAC). It is a follow-up of the 2LAC (Ackermann et al. 2011c) and makes use of the results of the 3FGL catalog (Fermi-LAT Collaboration 2015), a sequel to the 2FGL catalog (Nolan et al. 2012). The latter contained 1873 sources. In addition to dealing with more data, the 3FGL benefits from improved data selection, instrument response functions and analysis techniques. The 3FGL catalog includes 3033 sources with a Test Statistic ${ }^{72}$ (TS) greater than 25. Among them, 2192 sources are detected at $|b|>10^{\circ}$, where $b$ is the Galactic latitude. Among these $2192,1563(71 \%)$ are associated with high confidence with 1591 AGNs, which constitute the 3LAC. The 3LAC represents a sizeable improvement over the 2LAC as it includes $71 \%$ more sources ${ }^{73}$ (1591 versus 929) with an updated data analysis.

The paper is organized as follows. In Section 2, the observations by the LAT and the analysis employed to produce the four-year catalog are described. In Section 3, we explain the methods for associating gamma-ray sources with AGN counterparts and the different schemes for classifying 3LAC AGNs. Section 4 provides a brief census of the 3LAC sample and discusses sources of particular interest. Section 5 summarizes some of the properties of the 3LAC, including the gamma-ray flux distribution, the gamma-ray spectral properties, the redshift distribution, the gamma-ray luminosity distribution, and the gamma-ray variability properties. In Section 6, we address the connection with populations of blazars detected in the two neighboring energy bands, namely the hard X-ray and VHE bands. We discuss the implications of the 3LAC results in Section 7 and present our conclusions in Section 8 .

In the following, we use a $\Lambda \mathrm{CDM}$ cosmology with values from the Planck results (Planck Collaboration et al. 2014); in particular, we use $h=0.67, \Omega_{m}=0.32$, and $\Omega_{\Lambda}=0.68$, where the Hubble constant $H_{0}=100 h \mathrm{~km} \mathrm{~s}^{-1} \mathrm{Mpc}^{-1}$.

\section{OBSERVATIONS WITH THE LARGE AREA TELESCOPE_-ANALYSIS PROCEDURES}

The gamma-ray results used in this paper were derived in the context of the 3FGL catalog, so we only briefly summarize the analysis here and we refer the reader to the paper describing the 3FGL catalog (Fermi-LAT Collaboration 2015) for details. No additional analysis of the gamma-ray data was performed in the context of the present paper except for the fitting of the monthly light curves described in Section 5.5. The broadband spectral energy distribution (SED) fitting described in Section 3.1.2 was also carried out in this work.

The data were collected over the first 48 months of the mission, from 2008 August 4 to 2012 July 31 (MJD 54682 to 56139). Time intervals during which the rocking angle of the LAT was greater than $52^{\circ}$ were excluded and a cut on the zenith angle of gamma-rays of $100^{\circ}$ was applied to limit the contribution of Earth-limb gamma-rays. Time intervals with bright gamma-ray bursts and solar flares were excised. The

\footnotetext{
$\overline{72}$ We use the $\mathrm{TS}=2 \Delta \log L$ for quantifying how significantly a source emerges from the background, comparing the likelihood function $L$ with and without that source.

73 See Ackermann et al. (2015) for a 2LAC erratum. The corrected 2LAC full and clean samples include 929 and 827 sources, respectively. A total of 63 of the 88 sources mistakenly included in the initial 2LAC full sample are now in the 3 LAC catalog.
}

reprocessed Pass7REP_V15_Source event class was used, with photon energies between $100 \mathrm{MeV}$ and $300 \mathrm{GeV}$. This event class shows a narrower point-spread function above $3 \mathrm{GeV}$ than the Pass7 V6 Source class used in 2FGL. The source detection proce $\overline{d u r e}$ started with an initial set of sources from the 2FGL analysis: not just those reported in that catalog, but also including all candidates failing the significance threshold. With these seeds, an all-sky likelihood analysis produced an "optimized" model, where parameters characterizing the diffuse components, ${ }^{74}$ in addition to sources were fitted. The analysis of the residual TS map provided new seeds that were included in the model for a new all-sky likelihood analysis. This iterative procedure yielded over 4000 seeds that were then passed on to the maximum likelihood analysis for source characterization.

Events from the front and back sections of the LAT tracker (see Atwood et al. 2009, for details) were treated separately in the analysis. The analysis was performed with the binned likelihood method below $3 \mathrm{GeV}$ and the unbinned method above $3 \mathrm{GeV}$. These methods are implemented in the pyLikelihood library of the Science Tools ${ }^{75}$ (v9r23p0). Different spectral fits were carried out with a single power-law function $\left(d N / d E=N_{0}\left(E / E_{0}\right)^{-\Gamma}\right)$ and a log-parabola function $\left(d N / d E=N_{0}\left(E / E_{0}\right)^{-\alpha-\beta \log \left(E / E_{0}\right)}\right.$, Massaro et al. 2004), where $N_{0}$ is a normalization factor, $\Gamma, \alpha$ and $\beta$ are spectral parameters, and $E_{0}$ is an arbitrary reference energy adjusted on a source-by-source basis to minimize the correlation between $N_{0}$ and the other fitted parameters over the whole energy range $(0.1-300 \mathrm{GeV})$. Whenever the difference in $\log$ (likelihood) between these two fits was greater than 8 (i.e., $\mathrm{TS}_{\text {curve, }}$ which is defined as twice this difference, was greater than 16), the log-parabola results were retained. For 3C 454.3, an exponentially cutoff power law $\left(d N / d E=N_{0}\left(E / E_{0}\right)^{-\Gamma} \exp \left[\left(E_{0} / E_{c}\right)^{b}-\left(E / E_{c}\right)^{b}\right]\right.$, where $E_{c}$ is the cutoff energy and $b$ the exponential index) was needed to provide a reasonable fit to the data. The photon spectral index $(\Gamma)$ was obtained from the single power-law fit for all sources. A threshold of $\mathrm{TS}=25$, as calculated with the powerlaw model, was applied to all sources, corresponding to a significance of approximately $4 \sigma$. At the end of this procedure, 3033 sources survived the TS cut and constitute the 3FGL catalog.

Power-law fits were also performed in five different energy bands (100-300 MeV; $300 \mathrm{MeV}-1 \mathrm{GeV}$; 1-3 GeV; 3-10 GeV; 10-300 GeV), from which the energy flux was derived. A variability index $\left(\mathrm{TS}_{\mathrm{VAR}}\right)$ was constructed from a likelihood test based on the monthly averaged light curves, with the null (alternative) hypothesis corresponding to the source being steady (variable). A source is identified as being variable at the $99 \%$ confidence level if the variability index is equal or greater than 72.44 , TS $S_{\text {VAR }}$ being distributed as a $\chi^{2}$ function with 47 degrees of freedom.

Some of the 3FGL sources were flagged as doubtful when certain issues arose during their analyses (see 3FGL for a full list of these flags). The issues that most strongly affected the 3LAC list are: (i) sources with $\mathrm{TS}>35$ going down to TS $<25$ when changing the diffuse model, (ii) photon flux $(>1 \mathrm{GeV})$ or energy flux $(>100 \mathrm{MeV})$ changed by more than

\footnotetext{
74 The Galactic diffuse model and isotropic background model (including the gamma-ray diffuse and residual charged-particle backgrounds) are described in the 3FGL paper. Alternative Galactic diffuse models were tested as well.

75 http://fermi.gsfc.nasa.gov/ssc/data/analysis/documentation/Cicerone/
} 


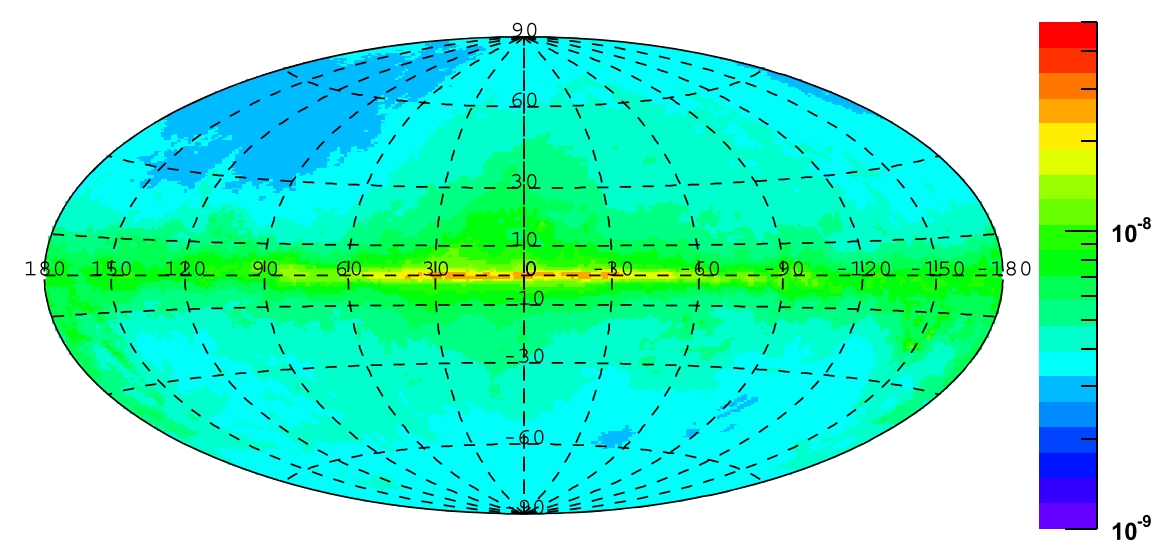

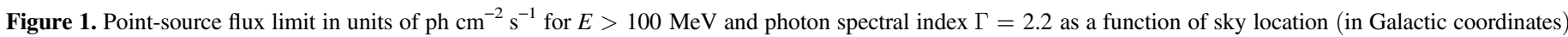
for the 3LAC time interval.
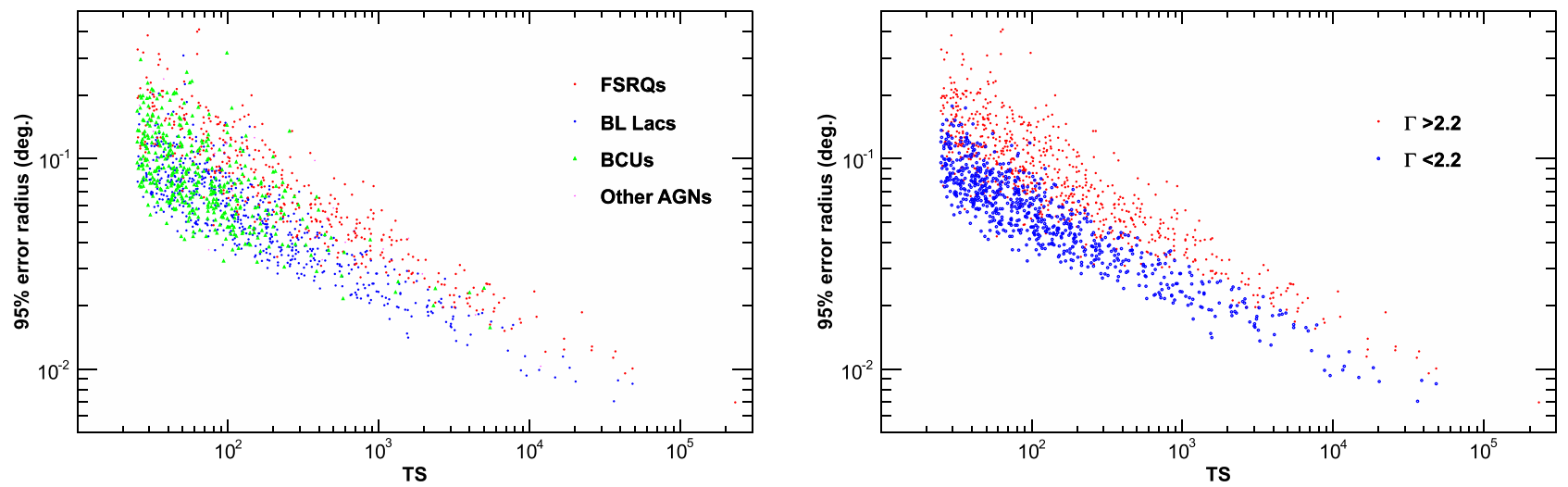

Figure 2. 95\% error radius vs. TS. Left: red circles: FSRQs, blue circles: BL Lacs, green triangles: unknown type (BCUs). Right: sources with $\Gamma>2.2$ (red) and $\Gamma<2.2$ (blue).

$3 \sigma$ and $35 \%$ when changing the diffuse model, (iii) sources located close to a brighter neighbor (the conditions are defined in Table 3 of 3FGL), and (iv) source Spectral_Fit_Quality $>16.3$ (Spectral_Fit_Quality is the $\chi^{2}$ between the fluxes in five energy bands and the spectral model). We developed a clean selection of sources by excluding sources that have any of the 3FGL analysis flags set. About 91\% (1444/1591) of the 3LAC sources survived this cut. Although the Spectral_Fit Quality condition may reject sources with unusual spectra, this condition ensures that the spectral properties discussed in the following are not affected by analysis issues.

A map of the LAT flux limit, calculated for the four-year period covered by this catalog, a TS $=25$, and a photon index of 2.2, is shown in Galactic coordinates in Figure 1. A map computed for a photon index of 1.8 would look very similar, with flux limits about four times lower. The $95 \%$ error radius, $\theta_{95}$, defined as the geometric mean of the semimajor and semiminor axes of the source location ellipse (see 3FGL), is plotted as a function of TS in Figure 2. It ranges from about 0.007 for $3 \mathrm{C} 454.3$, the brightest LAT blazar, to $0.08-0.3$ for sources just above the detection threshold depending on the gamma-ray spectral slope.

\section{SOURCE ASSOCIATION AND CLASSIFICATION}

In this work we look for candidate counterparts to 3FGL gamma-ray sources based on positional association with known cataloged objects that display AGN-type spectral characteristics. These characteristics are a flat radio spectrum between
1.4 and $5 \mathrm{GHz}$, an AGN-like broadband emission, core compactness or radio extended emission.

We recall here that in the context of AGNs, identification is only firmly established when correlated variability with a counterpart detected at other energies has been reported. So far, only 26 AGNs have met this condition (see 3FGL). For the rest, we use statistical approaches to find associations between LAT sources and AGNs. We will refer to the so-associated AGNs as the counterparts, although identification is not strictly established.

We apply the Bayesian Association Method (Abdo et al. 2010d) to catalogs of sources that were already classified and/or characterized. These catalogs come from specific instruments providing information on the spectrum and/or broadband emission. If a catalog reports an AGN classification, that is used. Otherwise the classification is made according to the criteria described below.

To broaden the possibility of associating a candidate AGN while knowing its broadband emission characteristics, we added the Likelihood Ratio (LR) Method (Ackermann et al. 2011c). This method can handle large uniform all-sky surveys and take the source space-density distribution into account. In the case of general radio or X-ray surveys, including AGN and non-AGN sources, the classification procedure is the same as for the Bayesian Association Method.

These two association approaches have been extensively described in previous catalog papers, so only updates will be given here (see Section 3.2). 


\subsection{Source Classification}

To define the criteria that a source must fulfill to be considered an AGN, the ingredients are primarily the optical spectrum and to a lesser extent other characteristics such as radio loudness, flat/steep radio spectrum between 1.4 and $5 \mathrm{GHz}$, broadband emission, flux variability, and polarization.

We stress that we are classifying the candidate counterpart to a 3FGL source. If available, the earlier classification in the literature of each reported candidate counterpart was checked.

\subsubsection{Optical Classification}

To optically classify a source we made use of different resources, in decreasing order of precedence: optical spectra from our intensive follow-up program (Shaw et al. 2013), the BZCAT list (i.e., classification from this list, which is a compilation of sources ever classified as blazars, Massaro et al. 2009), and spectra available in the literature, e.g., SDSS (Ahn et al. 2012), 6dF (Jones et al. 2009), when more recent than the version 4.1.1 of BZCAT (2012 August). The latter information was used only if we found a published spectrum.

The resulting classes are as follows.

1. Confirmed classifications: flat-spectrum radio quasar (FSRQ), BL Lac, radio galaxy, steep-spectrum radio quasar (SSRQ), Seyfert, and Narrow-Line Seyfert 1 (NLSy1)-these are sources with a well-established classification in the literature and/or through a well evaluated optical spectrum (with clear evidence for or lack of emission lines).

2. Tentative classifications: BCU-blazar candidates of uncertain type: these are considered candidate blazars because the association methods (see Sections 3.2.1 and 3.2.2) select a candidate counterpart that satisfies at least one of the following conditions:

(a) a BZU object (blazar of uncertain/transitional type) in the BZCAT list;

(b) a source with multiwavelength data in one or more of the WISE (D'Abrusco et al. 2013), AT20G (Murphy et al. 2010), VCS (Kovalev et al. 2007), CRATES (Healey et al. 2007), PMN-CA (Wright et al. 1996), CRATESGaps (Healey et al. 2007), or CLASS (Myers et al. 2003) source lists, that indicates a flat radio spectrum, and shows a typical two-humped, blazar-like SED; and

(c) a source included in radio and X-ray catalogs not listed above and for which we found a typical two-humped, blazar-like SED (see Böttcher 2007).

The $B C U$ sources are divided into three sub-types:

$B C U I$ : the counterpart has a published optical spectrum but is not sensitive enough for a classification as an FSRQ or a BL Lac;

$B C U$ II: the counterpart is lacking an optical spectrum but a reliable evaluation of the SED synchrotron-peak position is possible;

$B C U$ III: the counterpart is lacking both an optical spectrum and an estimated synchrotron-peak position but shows blazarlike broadband emission and a flat radio spectrum;

AGN-the counterparts show SEDs typical of radio-loud compact-core objects, but data are lacking in the literature to be more specific about their classes.

\subsubsection{SED Classification}

To better characterize the candidate counterparts of the 3FGL sources that we consider to be candidate blazars or more generally radio-loud AGNs, we studied their broadband SEDs by collecting all data available in the literature. ${ }^{76}$

We use the estimated value of the (rest-frame) broadbandSED synchrotron peak frequency $\nu_{\text {peak }}^{\mathrm{S}}$ to classify the source as either a low-synchrotron-peaked blazar (LSP, for sources with $\nu_{\text {peak }}^{\mathrm{S}}<10^{14} \mathrm{~Hz}$ ), an intermediate-synchrotron-peaked blazar (ISP, for $10^{14} \mathrm{~Hz}<\nu_{\text {peak }}^{\mathrm{S}}<10^{15} \mathrm{~Hz}$ ), or a high-synchrotronpeaked blazar (HSP, if $\nu_{\text {peak }}^{\mathrm{S}}>10^{15} \mathrm{~Hz}$ ). We refer the reader to the $2 \mathrm{LAC}$ paper for the list of broadband data used in this procedure.

The estimation of $\nu_{\text {peak }}^{\mathrm{S}}$ relies on a 3rd-degree polynomial fit of the low-energy hump of the SED performed on a source-bysource basis, while in previous catalogs (1LAC, 2LAC) an empirical parameterization of the SED based on the broadband indices $\alpha_{\text {ro }}$ (radio-optical) and $\alpha_{\text {ox }}$ (optical-X-rays) was used (see Abdo et al. 2010a). In this new method, some sources changed SED classification with respect to the 2LAC (see below).

This new procedure allows more objects to be assigned peak parameters than the empirical method since there is no need for a measured X-ray flux if the curvature is sufficiently pronounced in the IR-optical band. Even though a scrupulous check was performed for each individual source, caution is advised in using these $\nu_{\text {peak }}^{\mathrm{S}}$ values that were determined using non-simultaneous broadband data. Significant contamination from thermal/disk radiation may result in overestimation of the $\nu_{\text {peak }}^{\mathrm{S}}$ values of FSRQs, while the contribution of the host galaxy may bias the peak estimate toward lower frequencies in BL Lacs. Comparing the two procedures indicates that the new procedure leads to an average shift of +0.26 (rms: 0.49) and -0.05 (rms: 0.64) in $\log \nu_{\text {peak }}^{\mathrm{s}}$ relative to the previous one for FSRQs and BL Lacs, respectively, which we take as typical systematic uncertainties.

In the electronic tables, we report the so-obtained observerframe values of $\nu_{\text {peak }}^{\mathrm{S}}$, as well as the rest-frame values (i.e., corrected by a $(1+z)$ factor $)$. For BL Lac and BCU sources without measured redshifts, a redshift $z=0$ was assumed for the SED classification, but we omit these sources in figures showing $\nu_{\text {peak }}^{\mathrm{S}}$. Assuming a redshift of 1 for these sources as suggested by Giommi et al. (2013) would lead to a shift in the rest-frame $\log \nu_{\text {peak }}^{\mathrm{S}}$ of +0.3 , taken as an additional systematic uncertainty.

The $\nu_{\text {peak }}^{\mathrm{S}}$ distributions for FSRQs and BL Lacs are displayed in Figure 3. The FSRQ distribution is sharply peaked around $\log \nu_{\text {peak }}^{\mathrm{S}}=13$ while BL Lacs span the whole parameter space from low (LSP) to the highest frequencies (HSP). The BCU distribution resembles that of BL Lacs with an additional fairly weak component akin to FSRQs at this low $\nu_{\text {peak }}$ end.

\subsection{Source Association}

\subsubsection{The Bayesian Association Method}

This method (see Abdo et al. 2010d) uses Bayes' theorem to calculate the posterior probability that a catalog source is the

\footnotetext{
76 We made extensive use of the SED Builder online tool available at the ASI Science Data Center, http://tools.asdc.asi.it/SED/.
} 

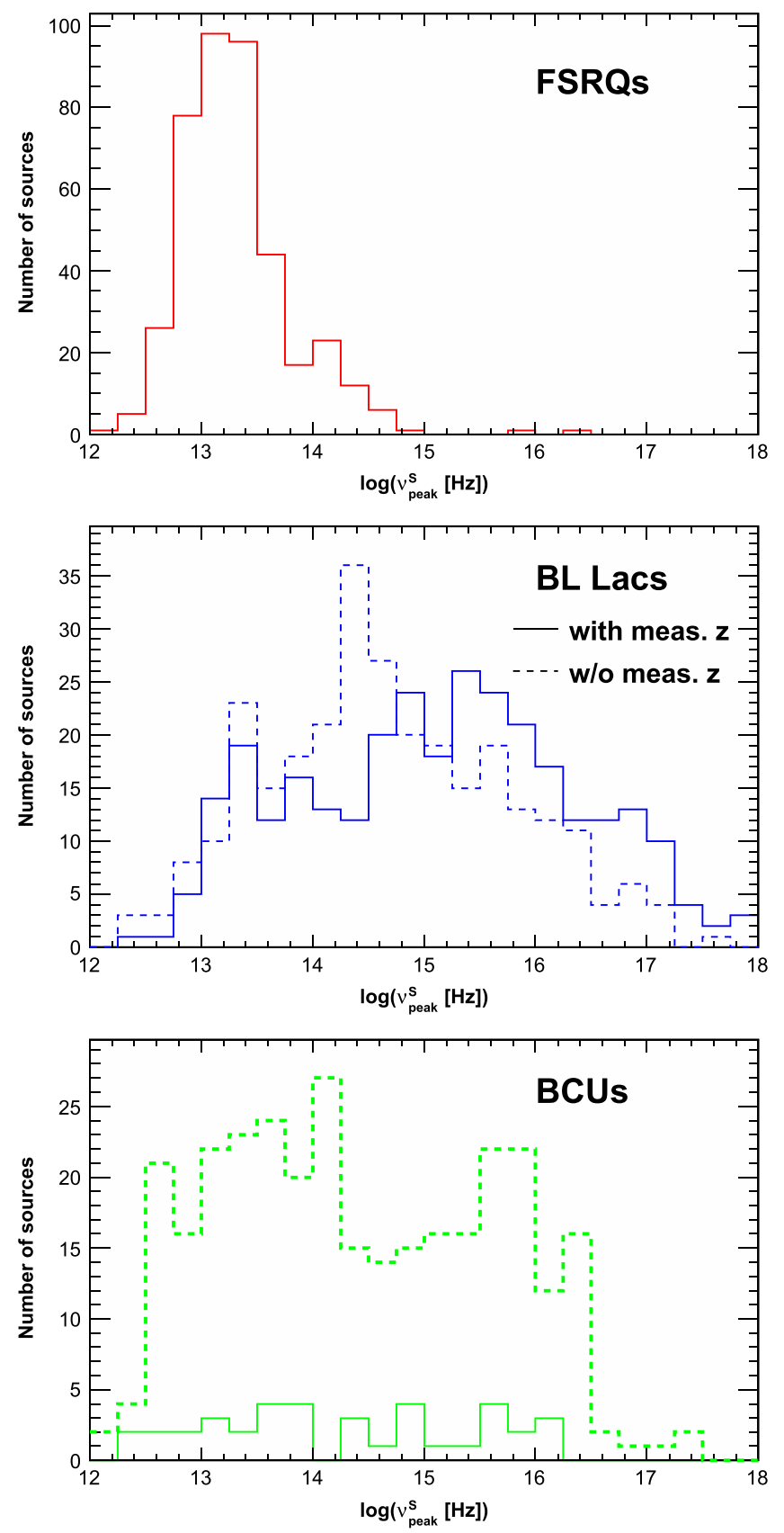

Figure 3. Distributions of the synchrotron peak frequency $\nu_{\text {peak }}^{\mathrm{S}}$ for FSRQs (top), BL Lacs (middle), and BCUs (bottom) in the Clean Sample (defined in Section 3.3). The solid and dashed histograms correspond to sources with and without measured redshifts, respectively. The $(1+z)$ correction factor (to convert to rest-frame values) has thus been applied to $\nu_{\text {peak }}^{\mathrm{S}}$ only for the former.

true counterpart of an LAT source. The significance of a spatial coincidence between a candidate counterpart from a catalog $\mathrm{C}$ and an LAT-detected gamma-ray source is evaluated by examining the local density of counterparts from $\mathrm{C}$ in the vicinity of the LAT source. If the candidate counterpart has not been established as an AGN in a catalog C, all we have is a positional association. The nature of the candidate counterpart is subsequently studied through the literature and SED study (See Section 3.1). The catalogs used in 3LAC are the 13th edition of the Veron catalog (Véron-Cetty \& Véron 2010), version 4.1.1 of BZCAT (Massaro et al. 2009), the CRATES and CGRaBs catalogs (Healey et al. 2007), the 2010 December 5 version of the VLBA Calibrator Source List, ${ }^{77}$ the most recent version of the TeVCat catalog ${ }^{78}$ and the Australia Telescope $20 \mathrm{GHz}$ Survey (AT20G; Murphy et al. 2010), which contains entries for 5890 sources observed at declination $\delta<0^{\circ}$. Associations with the Planck Early Release Catalogs (Planck Collaboration et al. 2011) were performed as well, but an association solely with a Planck counterpart was not considered sufficient to call the source an AGN candidate, as Planck detects sources of various types. Additions relative to 2LAC are the list of WISE gamma-ray blazar candidates from D'Abrusco et al. (2013) and Arsioli et al. (2015). The whole list of catalogs used in this method is given in Table 12 of the 3FGL paper (Fermi-LAT Collaboration 2015).

\subsubsection{The Likelihood-ratio Association Method}

The LR method has frequently been used to assess identification probabilities for radio, infrared, and optical sources (e.g., de Ruiter et al. 1977; Prestage \& Peacock 1983; Sutherland \& Saunders 1992; Lonsdale et al. 1998; Masci et al. 2001; Ackermann et al. 2011c). It is based on uniform surveys in the radio and in X-ray bands, enabling us to search for possible counterparts among the faint radio and X-ray sources. The LR makes use of counterpart densities (assumed spatially constant over the survey region) through the $\log N-\log S$ relation and therefore the source flux. As for the Bayesian method applied to catalogs without classification information, we can only claim a positional association for these counterparts. The nature of the candidate counterpart is subsequently studied through the literature and SED properties (see Section 3.1).

We made use of a number of relatively uniform radio surveys. Almost all radio AGN candidates of possible interest are in the NRAO VLA Sky Survey (NVSS; Condon et al. 1998), and the Sydney University Molonglo Sky Survey (SUMSS; Mauch et al. 2003). We also added AT20G. In this way we are able to look for radio counterparts with detections at higher frequencies. To look for additional possible counterparts we cross-correlated the LAT sources with the most sensitive all-sky X-ray survey, the ROSAT All Sky Survey (RASS) Bright and Faint Source Catalogs (Voges et al. 1999, 2000). The method, which computes the probability that a suggested association is the "true" counterpart, is described in detail in Section 3.2 of the 2LAC paper. A source is considered a likely counterpart of the gamma-ray source if its reliability, $\log \mathrm{LR}$, (see Equation (4) in the 2LAC paper) is greater than 0.8 in at least one survey. The critical values of $\log L_{\mathrm{c}}$ above which the reliability is greater than 0.8 are $1.69,0.52,2.42$, and 5.80 for the NVSS, SUMSS, RASS, and AT20G surveys, respectively.

\subsection{Association Results}

The adopted threshold for the association probability is 0.80 in either method. This value represents a compromise between association efficiency and purity. As in previous LAC catalog versions, we define a Clean Sample as 3LAC single-association sources free of the analysis issues mentioned in Section 2. Table 1 compares the performance of the two methods in terms

\footnotetext{
77 The VLBA Calibrator Source List can be downloaded from http://www. vlba.nrao.edu/astro/calib/vlbaCalib.txt.

78 http://tevcat.uchicago.edu
} 
Table 1

Comparison of Association Methods in Terms of the Total Number of Associations, the Estimated Number of False Associations $\left(N_{\text {false }}\right)$, and the Number of Sources Associated only via a Given Method, $N_{\mathrm{S}}$

\begin{tabular}{lcccccrr}
\hline \hline Sample & \multicolumn{2}{c}{ All Methods } & \multicolumn{3}{c}{ Bayesian Method } & \multicolumn{2}{c}{ LR Method } \\
& Total & $N_{\text {false }}$ & Total & $N_{\text {false }}$ & $N_{\mathrm{S}}$ & Total & $N_{\text {false }}$ \\
\hline All & 1591 & 29.7 & 1529 & 34.5 & 379 & 1212 & 120.5 \\
Clean Sample & 1444 & 23.4 & 1391 & 17.5 & 337 & 1107 & 107.3 \\
\hline
\end{tabular}

of the total number of associations, the estimated number of false associations $N_{\text {false, }}$, calculated as $N_{\text {false }}=\sum_{i}\left(1-P_{i}\right)$, where $P_{i}$ is the association probability for the $i$ th source, and the number of sources associated solely via a given method, $N_{\mathrm{S}}$, for the full and Clean samples.

The fraction of sources associated by both methods is $71 \%$ (1150/1591), 379, and 62 sources being solely associated with the Bayesian and LR methods, respectively. Among the former, 177 sources are associated due to the list of WISE gamma-ray blazar candidates only (over 1000 3FGL sources have counterparts in that catalog). The overall false-positive rate is $1.9 \%$. The estimated number of false positives among the 571 sources not previously detected in 2FGL and previous LAT catalogs is $12.0(2.1 \%)$.

Figure 4 displays the distributions of separation distance between the gamma-ray sources and their assigned counterparts, normalized to $\sigma=\theta_{95} / \sqrt{-2 \log (0.05)}$, for the whole sample and for the newly detected sources. Both agree well with the distributions expected for real associations, as expected from the overall low false-positive rate.

\subsection{Blazar Candidates by the Australia Telescope Compact Array}

In this section, we point out blazar candidates derived from the recent work of Petrov et al. (2013) but not all included in 3LAC. Using the Australia Telescope Compact Array (ATCA) at $5 \mathrm{GHz}$ and $9 \mathrm{GHz}$, Petrov et al. (2013) detected 424 sources in the LAT error ellipses of southern unassociated 2FGL sources. They found that 84 of them have radio-source counterparts with a spectral index flatter (i.e., greater) than -0.5 .

The 424 sources are characterized by weak radio fluxes $(<100 \mathrm{mJy})$, and were thus missing from the previous AT20G. Flat spectrum radio sources cannot be directly associated with extragalactic sources like blazars, as peculiar Galactic objects (like, for example, $\eta$ Carinae, microquasars, compact $\mathrm{H}_{\text {II }}$ regions, planetary nebulae) can also exhibit a flat radio spectrum. On the other hand a steep radio spectrum does not rule out an extragalactic nature. A total of 24 sources among the 84 flat-spectrum ones are included in 3LAC, as they now fulfill the required criterion (association probability greater than 0.8). An additional 21 sources listed in Table 2 show double-humped radio-to-gamma-ray SEDs resembling those of BCU, but they have association probabilities below threshold. More data may help secure these associations in the future.

\section{THE THIRD LAT AGN CATALOG (3LAC)}

\subsection{Census}

Table 3 summarizes the 3LAC source statistics. The 3LAC includes 1591 objects, with 467 FSRQs, 632 BL Lacs, 460

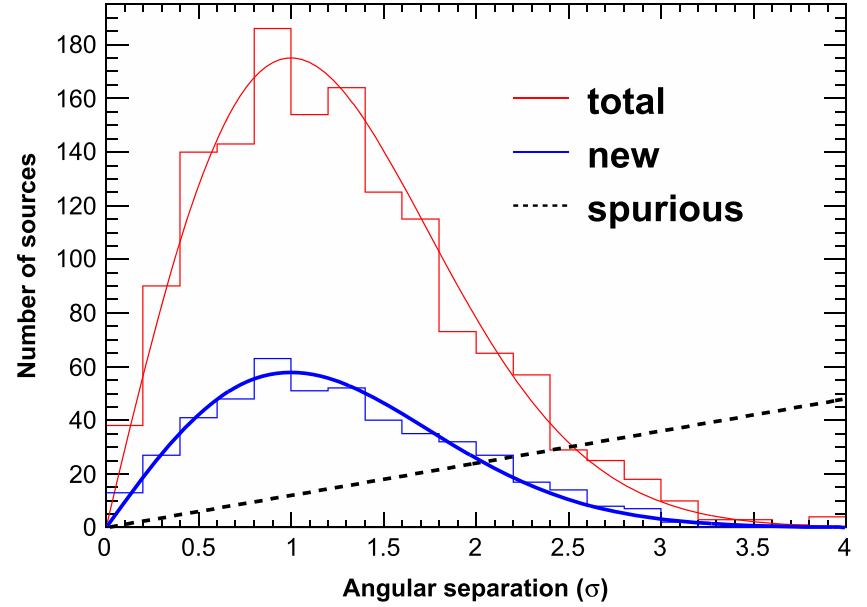

Figure 4. Distributions of normalized angular separation between 3LAC sources and their assigned counterparts. The normalization factor $\sigma$ is defined in the text. Red: total. Blue: new sources. The curves correspond to the expected distribution for real associations and the dashed line illustrates that expected for spurious associations.

BCUs, and 32 non-blazar AGNs. Their properties are given in Table 4

A total of 1563 gamma-ray sources have been associated with radio-loud AGNs among $2192|b|>10^{\circ}$ 3FGL sources, corresponding to an overall association fraction of $72 \%$. The fraction changes substantially between the northern and southern celestial hemispheres $(843 / 1136=74 \%$ and $731 /$ $1056=69 \%$ respectively), an effect essentially entirely driven by unassociated southern-hemisphere BL Lacs as discussed below.

Only sources in the Clean Sample will be used in the following tallies and figures unless stated otherwise. It includes 1444 objects with 414 FSRQs, 604 BL Lacs, 402 BCUs, and 24 non-blazar AGNs.

A comparison of the results inferred from the 3LAC and 2LAC enables the following observations:

1. The 3LAC Clean Sample includes 619 more sources than the 2LAC Clean Sample, i.e., a 75\% increase. Of these, 477 sources are new (81 FSRQs, 146 BL Lacs, 240 blazars of unknown type, 10 non-blazar objects); the other sources were present in previous Fermi catalogs but not included in Clean Samples for various reasons (e.g., the corresponding gamma-ray sources were not associated with AGNs, had more than one counterpart or were flagged in the analysis). The fraction of new sources (not present in 1FGL or 2FGL) is slightly higher for hardspectrum (i.e., $\Gamma<2.2$ ) sources than for soft-spectrum ones (i.e., $\Gamma>2.2$ ), $51 \%$ versus $47 \%$, respectively, but the relative increase reaches $72 \%$ for very hard-spectrum (i.e., $\Gamma<1.8$ ) sources. 
Table 2

List of ATCA Blazar Candidates

\begin{tabular}{|c|c|c|c|c|c|c|}
\hline 3FGL Name & Counterpart name & $\begin{array}{l}\text { R.A. radio } \\
\left({ }^{\circ}\right)\end{array}$ & $\begin{array}{c}\text { Decl. radio } \\
\left({ }^{\circ}\right)\end{array}$ & Class count & $\log \left(\nu_{\text {peak }}^{\mathrm{S}}[\mathrm{Hz}]\right)$ & 2FGL Name \\
\hline $\mathrm{J} 0102.1+0943$ & NVSS J010217+094407 & 15.57133 & 9.73622 & BCU II & 14.419 & J0102.2+0943 \\
\hline J0437.7-7330 & SUMSS J043836-732921 & 69.65392 & -73.48994 & BCU III & $\ldots$ & J0438.0-7331 \\
\hline J0725.7-0550 & NVSS J072547-054832 & 111.44867 & -5.80753 & BCU III & $\ldots$ & J0725.8-0549 \\
\hline J0737.8-8245 & SUMSS J073706-824836 & 114.47621 & -82.73703 & BCU III & $\cdots$ & J0737.5-8246 \\
\hline J0937.9-1435 & NVSS J093754-143350 & 144.47783 & -14.56414 & BCU II & 17.150 & J0937.9-1434 \\
\hline J1016.6-4244 & 1RXS J101620.6-424733 & 154.08650 & -42.78975 & BCU II & 15.600 & J1016.4-4244 \\
\hline J1038.0-2425 & NVSS J103824-242355 & 159.59987 & -24.39869 & BCU II & 12.550 & J1038.2-2423 \\
\hline J1117.2-5338 & MGPS J111715-533816 & 169.31279 & -53.63783 & BCU II & 14.755 & J1117.2-5341 \\
\hline J1115.0-0701 & NVSS J111511-070238 & 168.79832 & -7.04417 & BCU III & $\ldots$ & J1115.0-0701 \\
\hline J1207.2-5052 & SUMSS J120719-505350 & 181.79211 & -50.86061 & BCU III & $\ldots$ & J1207.3-5055 \\
\hline J1240.3-7149 & MGPS J124021-714901 & 190.08821 & -71.81653 & BCU III & $\cdots$ & J1240.6-7151 \\
\hline J1249.1-2808 & NVSS J124919-280833 & 192.33118 & -28.14239 & BCU II & 15.080 & J1249.5-2811 \\
\hline $\mathrm{J} 1424.3-1753$ & NVSS J142412-175010 & 216.05145 & -17.83611 & BCU II & 15.750 & $\mathrm{~J} 1424.2-1752$ \\
\hline J1539.2-3324 & NVSS J153911-332209 & 234.79825 & -33.36822 & BCU III & $\ldots$ & J1539.2-3325 \\
\hline $\mathrm{J} 1704.4-0528$ & NVSS J170433-052839 & 256.14075 & -5.47753 & BCU II & 15.200 & J1704.6-0529 \\
\hline $\mathrm{J} 1747.3+0324$ & NVSS J174733+032703 & 266.88860 & 3.45119 & BCU III & $\cdots$ & $\mathrm{J} 1747.6+0324$ \\
\hline J1757.7-6030 & SUMSS J175734-603032 & 269.39413 & -60.50794 & BCU III & $\ldots$ & $\mathrm{J} 1757.5-6028$ \\
\hline J2034.6-4202 & SUMSS J203451-420024 & 308.71274 & -42.01044 & BCU II & 15.640 & $J 2034.7-4201$ \\
\hline $\mathrm{J} 2046.7-4259$ & SUMSS J204643-425711 & 311.68353 & -42.95358 & BCU III & $\ldots$ & $\mathrm{J} 2046.2-4259$ \\
\hline $\mathrm{J} 2134.5-2131$ & NVSS J213430-213032 & 323.62580 & -21.50858 & BCU II & 15.410 & $\mathrm{~J} 2134.6-2130$ \\
\hline $\mathrm{J} 2258.2-3645$ & NVSS J225815-364433 & 344.56195 & -36.74264 & BCU II & 15.150 & $\mathrm{~J} 2257.9-3646$ \\
\hline
\end{tabular}

2. The fraction of BCU has increased notably between the two catalogs (from 20\% to 28\%). The number of these sources in the 3LAC Clean Sample has increased by more than a factor of 2.5 relative to that in the 2LAC Clean Sample, being almost equal to the number of FSRQs. This increase is mainly due to the lower probability of having a published high-quality spectrum available for these fainter sources because of the lack of optical/nearinfrared observing programs. The census of the BCU sources in the Clean Sample is: 49 BCU I, 308 BCU II, 45 BCU III.

3. The relative increase in BCUs drives a drop in the proportions of FSRQs and BL Lacs, which only represent $29 \%$ and $41 \%$ of the 3LAC Clean Sample, respectively (38\% and $48 \%$ for $2 \mathrm{LAC}$ ). The relative increase in the number of sources with respect to $2 \mathrm{LAC}$ is $34 \%$ and $42 \%$ for FSRQs and BL Lacs respectively.

4. Out of 827 sources in the 2LAC Clean Sample, a total of 69 are missing in the 3LAC Clean Sample (42 in the full sample), some of them probably due to variability effects. A few others are present in 3FGL but with shifted positions, ruling out their association with their former counterparts.

The loci of sources in the Clean Sample are shown in Figure 5, both in Galactic and celestial coordinates. The deficit in classified AGNs in the region of the celestial south pole already reported in $2 \mathrm{LAC}$ is clearly visible, while a relative excess is seen in the region of the celestial north pole. This anisotropy is mainly driven by BL Lacs, with $51 \%$ more sources in the northern Galactic hemisphere (362) than in the southern one (242). This effect is ascribed to the relative incompleteness of the counterpart catalogs in the southern hemisphere (for instance, NVSS only covers the $\delta>-40^{\circ}$ sky, where $\delta$ is the declination). It is very partially offset by an observed relative excess (54 sources) of associations with BCU in the south relative to the north.

\subsection{Non-blazar Objects and Misaligned AGNs}

Blazars represent the overwhelming majority of 3LAC AGNs, with non-blazar AGNs only constituting $2 \%$ of the sample. In 2LAC, eleven sources were classified as AGNs, i.e., were neither confirmed blazars nor blazar candidates (such as BCUs). Although there may have been evidence for their flatness in radio emission or broadband emission, our intensive optical follow-up program did not provide clear evidence for optical blazar characteristics. Nine of them remain in 3LAC, and are now all classified as BCUs, except for one now classified as a BL Lac.

Misaligned AGNs (MAGNs), with jets pointing away from the observer, are not favored $\mathrm{GeV}$ sources. By MAGNs we mean radio-loud AGNs with jets directed at large angles relative to the line-of-sight that display steep radio spectra $\left(\alpha_{r} \geqslant 0.5\right.$, with the usual convention that $\left.F_{\nu} \propto \nu^{-\alpha}\right)$ and bipolar or quasi-symmetrical structures in radio maps. The larger jet inclination angle relative to blazars means the observed radio emission from the relativistic jet is not significantly Doppler boosted, making it less prevalent over other radio components such as synchrotron radiation from mildly relativistic outflows or extended radio lobe emission (Abdo et al. 2010e).

Table 5 summarizes the non-blazar objects and MAGNs in the $3 \mathrm{FGL} / 3 \mathrm{LAC}$, also noting their previous appearances in the 2FGL/2LAC and 1FGL/1LAC. All the 1FGL sources, detected in 11 months of exposure, were subsequently studied with 15 months of data (Abdo et al. 2010e).

M 87 was one of the first new Fermi-LAT detections (Abdo et al. 2009d) of a source classified as a non-blazar object, being a low-power Fanaroff \& Riley (1974) type-I (FRI) radio galaxy. Many of the newly associated non-blazar objects are nearby FRIs-J0758.7+3747 (3C 189, a.k.a., B2 0755+37) and $3 \mathrm{C}$ 264. The gamma-ray detection of the latter case was recently reported in a study of its parent cluster Abell 1367 (Ackermann et al. 2011a), although the gamma-rays likely originate from the AGN. We remark that 0!8 away from 3C 
Table 3

Census of Sources

\begin{tabular}{|c|c|c|c|}
\hline AGN Type & $\begin{array}{l}\text { Entire } \\
\text { 3LAC }\end{array}$ & $\begin{array}{l}\text { 3LAC Clean } \\
\text { Sample }^{\mathrm{a}}\end{array}$ & $\begin{array}{l}\text { Low-latitude } \\
\text { Sample }\end{array}$ \\
\hline$\overline{\text { All }^{\mathrm{b}}}$ & 1591 & 1444 & 182 \\
\hline FSRQ & 467 & 414 & 24 \\
\hline ...LSP & 412 & 366 & 24 \\
\hline ...ISP & 47 & 42 & 0 \\
\hline$\ldots$..HSP & 3 & 2 & 0 \\
\hline ...no SED classification & 5 & 4 & 0 \\
\hline BL Lac & 632 & 604 & 30 \\
\hline ..LSP & 162 & 150 & 8 \\
\hline$\ldots$..ISP & 178 & 173 & 6 \\
\hline ...HSP & 272 & 265 & 12 \\
\hline ...no SED classification & 20 & 16 & 4 \\
\hline Blazar of Unknown type & 460 & 402 & 125 \\
\hline$\ldots$ BCU I & 57 & 49 & 11 \\
\hline ...LSP BCU I & 26 & 24 & 8 \\
\hline ...ISP BCU I & 11 & 9 & 1 \\
\hline ...HSP BCU I & 13 & 13 & 2 \\
\hline $\begin{array}{l}\text {...BCU I w/o SED } \\
\text { classification }\end{array}$ & 7 & 3 & 0 \\
\hline$\ldots \mathrm{BCU}$ II & 346 & 308 & 85 \\
\hline ...LSP BCU II & 156 & 129 & 39 \\
\hline ...ISP BCU II & 78 & 70 & 13 \\
\hline ...HSP BCU II & 107 & 105 & 31 \\
\hline $\begin{array}{l}\text {...BCU II w/o SED } \\
\text { classification }\end{array}$ & 5 & 4 & 2 \\
\hline$\ldots$ BCU III & 57 & 45 & 29 \\
\hline ...LSP BCU III & 16 & 11 & 9 \\
\hline ...ISP BCU III & 0 & 0 & 0 \\
\hline ...HSP BCU III & 0 & 0 & 0 \\
\hline $\begin{array}{l}\text {...BCU III w/o SED } \\
\text { classification }\end{array}$ & 41 & 34 & 20 \\
\hline Non-blazar AGN & 32 & 24 & 3 \\
\hline$\ldots \mathrm{CSS}$ & 2 & 1 & 0 \\
\hline ....NLSy1 & 5 & 5 & 0 \\
\hline$\ldots$ RG & 14 & 13 & 2 \\
\hline ...SSRQ & 5 & 3 & 0 \\
\hline ...Other AGN & 6 & 2 & 1 \\
\hline
\end{tabular}

Notes.

${ }^{\text {a }}$ Sources with single counterparts and without analysis flags. See Section 3.1 for the definitions of this sample.

${ }^{\mathrm{b}}$ Bold values are the total numbers for each subclass.

189 lies the quasar SDSS J075825.87+374628.7 with redshift 1.50. With the resolution of the NVSS, this source cannot be disentangled from the radio emission of 3C 189. This may be the reason why this source is not present in the NVSS catalog, precluding the estimation of the association probability with the gamma-ray source.

NGC 1275 (3C 84, Perseus A) was first detected in the initial LAT bright source list based on 3 months of data (Abdo et al. 2009e). It was probably detected previously with COS-B (Strong \& Bignami 1983), but not with EGRET. In the Fermi era, it is a strong source, exhibiting GeV variability (Abdo et al. 2009c; Kataoka et al. 2010). 3C 120 is not listed in any of the FGL catalogs but its detection was reported in a 15 month study (Abdo et al. 2010e). There are indications that 3C 120 undergoes a series of flares with a low long-term average flux. For instance, in 2014 September a flaring source positionally consistent with 3C 120 was detected with a high significance (TS > 50; Tanaka et al. 2014). The closest 3FGL source, 3FGL J0432.5+0539, lies 0.35 away, with an $95 \%$ error radius of 0.15 , hampering association with $3 \mathrm{C} 120$ by our methods. This gamma-ray source has a soft spectrum $(\Gamma=2.7 \pm 0.1)$, comparable with that ascribed to 3C 120 (Abdo et al. 2010e; Kataoka et al. 2011). The possibility of two separate, softspectrum sources cannot be excluded. Another known example from previous lists is 3C 78 (NGC 1218; Abdo et al. 2010g).

Cen A was also reported in the initial LAT bright source list (Abdo et al. 2009e), confirming the EGRET source (Hartman et al. 1999; Sreekumar et al. 1999). It remains as the only AGN with a significant detection of extended gamma-ray emission (Abdo et al. 2010b). There is no convincing case of extended emission in other radio galaxies with relatively large radio extensions, such as Cen B (Katsuta et al. 2013), NGC 6251 (Takeuchi et al. 2012), and Fornax A. Fornax A may be a good case to investigate this emission (Cheung et al. 2007; Georganopoulos et al. 2008). The closest 3FGL source is offset from the Fornax A core by 0.15 , while the $95 \%$-contour distance is 0.092 (see Figure 6 for a VLA $1.5 \mathrm{GHz}$ image). NGC 6251 (one square degree in solid angle) was also detected by EGRET (Mukherjee et al. 2002). Its location shifted between $1 \mathrm{LAC}$ and $2 \mathrm{LAC}$ toward the western radio lobe.

3C 111 was also a previous EGRET source (Hartman et al. 2008) and also shows apparent variability (e.g., Abdo et al. 2010e; Kataoka et al. 2011; Grandi et al. 2012). It joins the other two FR type-II sources listed in Table 5: 3FGL $\mathrm{J} 1442.6+5156$ (3C 303) and 3FGL J0519.2-4542 (Pictor A). The latter are also broad-lined radio galaxies (BLRGs), and are new detections. The LAT detection of Pictor A was reported by Brown \& Adams (2012) following ${ }^{79}$ a previous tentative detection (Kataoka et al. 2011).

The previous LAT detections of PKS 0625-35 and IC 310, two radio galaxies with BL Lac characteristics, were reported in 2LAC, and are confirmed. IC 310 has been classified as a head-tail galaxy (Neronov et al. 2010), but recent works have found increasing evidence for blazar-like properties, e.g., blazar-like VLBI jet structure (Kadler et al. 2012a) and extremely fast $\mathrm{TeV}$ variability (Aleksić et al. 2014). The source 4C +39.12 (3FGL J0334.2+3915) was classified as a lowpower compact source by Giovannini et al. (2001), separate from its Fanaroff-Riley classification. Two new compact steepspectrum (CSS) sources are detected: 3FGL J1330.5+3023 (3C 286) and 3FGL J0824.9+3916 (4C +39.23B). While both are CSS, the latter is a duplicate association (the other association being the FSRQ blazar $4 \mathrm{C}+39.23$ ) so it is not in the Clean Sample. The former has the morphology of a medium symmetric object (MSO), like that of the LAT-detected FSRQ 4C +55.17 (McConville et al. 2011).

The gamma-ray detections of 3C 207 and 3C 380 were first reported in 1LAC. They appear in the 3CRR catalog (Laing et al. 1983) by virtue of their bright low-frequency emission due to the presence of kpc-scale extended steep-spectrum radio lobes, and thus are formally classified as SSRQs. However, they contain pronounced flat-spectrum radio cores with

\footnotetext{
79 The results of Brown \& Adams (2012) are in tension with those presented here, with their reported statistical uncertainties being very small given the low source flux.
} 
Table 4

High Latitude $\left(|b|>10^{\circ}\right)$ 3LAC Full Sample

\begin{tabular}{|c|c|c|c|c|c|c|c|c|c|c|c|c|c|c|}
\hline $\begin{array}{l}\text { 3FGL Source } \\
\text { Name } \\
\text { (1) }\end{array}$ & $\begin{array}{l}\text { Counterpart } \\
\text { Name } \\
(2)\end{array}$ & $\begin{array}{l}\text { R.A. } \\
\left({ }^{\circ}\right) \\
(3)\end{array}$ & $\begin{array}{c}\text { Decl. } \\
\left({ }^{\circ}\right) \\
(4)\end{array}$ & $\begin{array}{c}\text { AngSep } \\
\left({ }^{\circ}\right) \\
(5)\end{array}$ & $\begin{array}{l}\theta_{95} \\
\left({ }^{\circ}\right) \\
(6)\end{array}$ & $\begin{array}{l}\text { Optical } \\
\text { Class } \\
(7)\end{array}$ & $\begin{array}{l}\text { SED } \\
\text { Class } \\
(8)\end{array}$ & $\begin{array}{c}\log \left(\nu_{\text {peak }}^{\text {S,meas }}\right) \\
(9)\end{array}$ & $\begin{array}{c}\log \left(\nu_{\text {peak }}^{\mathrm{S}}\right) \\
(10)\end{array}$ & $\begin{array}{c}z \\
(11)\end{array}$ & $\begin{array}{l}\text { Prob. } \\
\text { Bay. } \\
(12)\end{array}$ & $\begin{array}{c}\text { Rel. } \\
\text { LR }_{\mathrm{RG}} \\
(13)\end{array}$ & $\begin{array}{c}\text { Rel. } \\
\text { LR }_{X G} \\
(14)\end{array}$ & $\begin{array}{c}\text { Compton } \\
\text { Dominance } \\
\text { (15) }\end{array}$ \\
\hline $\mathrm{J} 0001.2-0748^{\mathrm{a}}$ & PMN J0001-0746 & 0.32510 & -7.77411 & 0.042 & 0.075 & BLL & ISP & 14.486 & 14.486 & $\cdots$ & 0.9978 & 0.859 & 0.00 & $\cdots$ \\
\hline $\mathrm{J} 0001.4+2120^{\mathrm{a}}$ & TXS 2358+209 & 0.38502 & 21.22679 & 0.113 & 0.199 & FSRQ & ISP & 14.163 & 14.486 & 1.10600 & 0.924 & 0.00 & 0.000 & 2.40 \\
\hline $\mathrm{J} 0002.2-4152^{\mathrm{a}}$ & 1RXS J000135.5-415519 & 0.38642 & -41.92367 & 0.137 & 0.174 & BCU II & HSP & 15.800 & 15.800 & $\cdots$ & 0.972 & 0.000 & 0.000 & -0.59 \\
\hline $\mathrm{J} 0003.2-5246^{\mathrm{a}}$ & RBS 0006 & 0.83121 & -52.79103 & 0.017 & 0.065 & BCU II & HSP & 16.850 & 16.850 & $\cdots$ & 0.998 & 0.000 & 0.900 & -0.52 \\
\hline J0003.8-1151 & PMN J0004-1148 & 1.02048 & -11.81622 & 0.076 & 0.114 & BCU II & LSP & 12.515 & 12.515 & $\cdots$ & 0.995 & 0.869 & 0.000 & $\cdots$ \\
\hline J0003.8-1151 & PKS 0001-121 & 0.92848 & -11.86372 & 0.030 & 0.114 & BCU I & $\ldots$ & $\ldots$ & $\ldots$ & 1.30999 & 0.988 & 0.871 & 0.000 & $\ldots$ \\
\hline
\end{tabular}

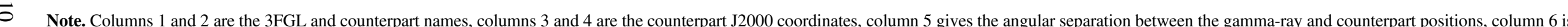

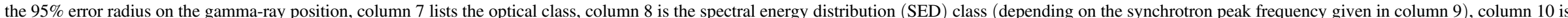

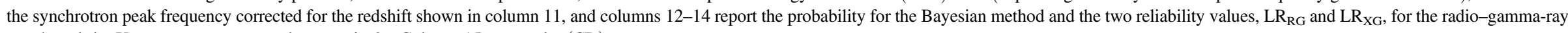
match and the X-ray-gamma-ray match respectively. Column 15 reports $\log (\mathrm{CD})$.

${ }^{a}$ Refers to sources in the Clean Sample.

(This table is available in its entirety in machine-readable form.) 

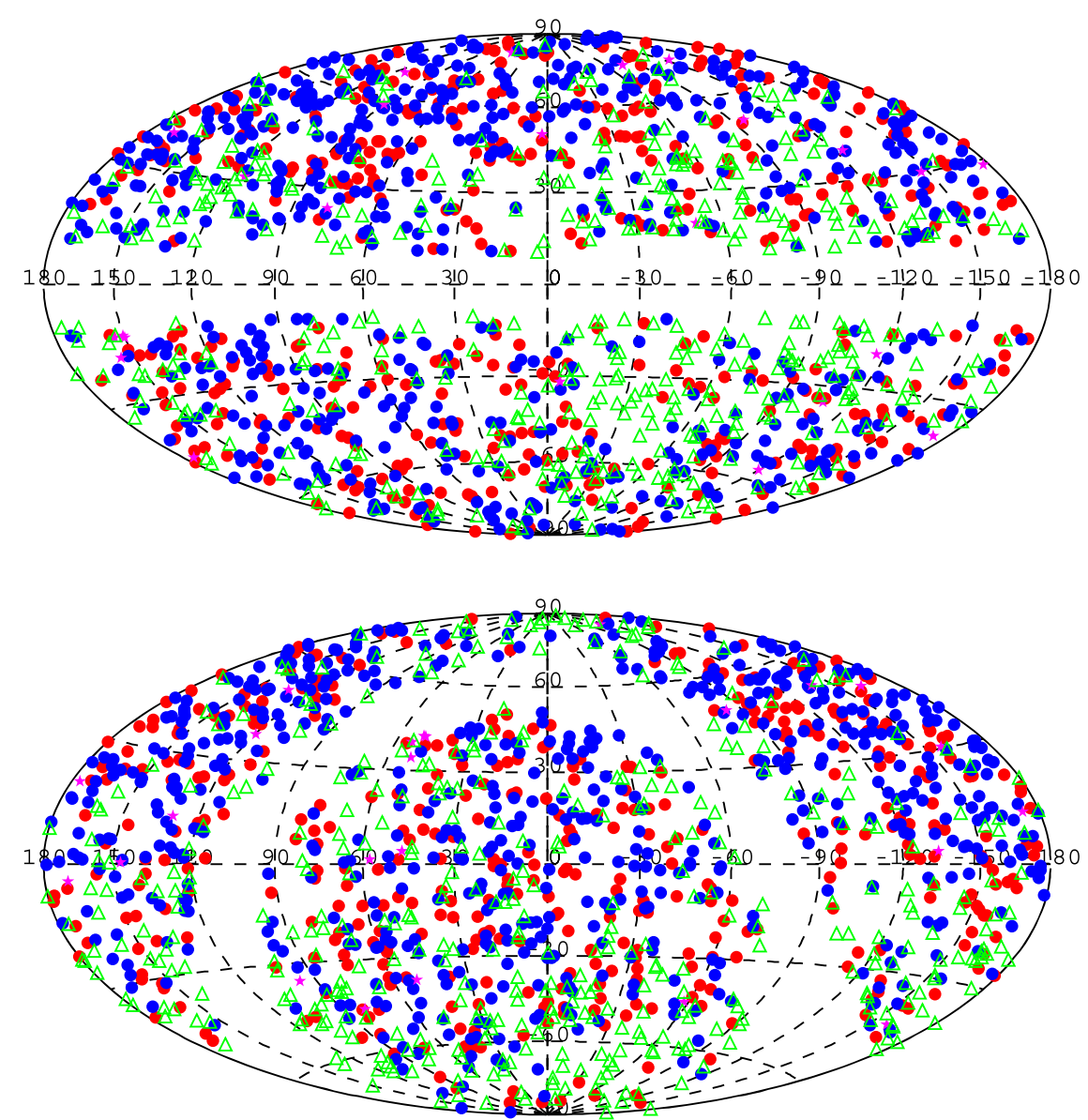

Figure 5. Locations of the sources in the Clean Sample in Galactic (top) and J2000 equatorial (bottom) coordinates. Red circles: FSRQs; blue circles: BL Lacs; green triangles: blazars of unknown type; magenta stars: other AGNs.

superluminal motions measured in their parsec-scale jets, indicating that they are the most well-aligned sources to our line of sight among the SSRQs in the 3CRR (e.g., Wilkinson et al. 1991; Hough 2013; Lister et al. 2013). New ones to highlight are 3C 275.1 (3FGL J1244.1+1615), TXS 0348+013 (3FGL J0351.1+0128), and 4C +39.26 (3FGL J0934.1+3933). The SSRQ 4C+04.40 is part of a double association (with the FSRQ MG1 J120448+0408) of 3FGL J1205.4+0412.

GB $1310+487$ is a gamma-ray/radio-loud narrow-line AGN at $z=0.638$, showing a gamma-ray flare in November 2009 and located behind the disk of an unrelated emission-line galaxy at $z=0.500$ (Sokolovsky et al. 2014).

Circinus, a type-2 Seyfert galaxy located at $b=-3: 8$ and thus not in 3LAC, was recently detected (Hayashida et al. 2013). Other Seyfert detections were investigated (Teng et al. 2011; Ackermann et al. 2012b; Lenain et al. 2010), but were found to be starburst galaxies (Ackermann et al. 2012a).

The detections of NGC 6951 (classified as a Seyfert 2 galaxy and a LINER, reported in 1LAC but missing in 2LAC), 3C 407 (a source with broad emission lines but with a fairly steep radio spectrum and reported in 2LAC), and NGC 6814 (type 1.5 Seyfert galaxy, also reported in 2LAC) are not confirmed. The same conclusion applies to PKS 0943-76 (studied in Abdo et al. 2010e). The previous claim that it has a FRII morphology was based on a low-resolution radio map from Burgess \& Hunstead (2006). The offset between the 4 year source and PKS $0943-76$ is 0.22 , while the radius of the source location region at the $95 \%$ confidence level is 0.12 . ESO $323-\mathrm{G} 77$ (type 2 Seyfert galaxy), and PKS0943-76 (radio galaxy), both reported in $2 \mathrm{LAC}$, were actually both mis-associated because of an error in the LR association method (Ackermann et al. 2015).

Five sources are associated with NLSy1. Four of them were included in 2LAC: 3FGL J0325.2+3410 (BZU J0324+3410), 3FGL J0948.8+0021 (PMN J0948+0022), 3FGL J1505.1 +0326 (BZQ J1505+0326), and 3FGL J2007.8-4429 (BZQ J2007-4434), while 3FGL 0849.9+5108 (SBS 0846+513) was first reported by Donato \& Perkins (2011) and further studied by D'Ammando et al. $(2012,2013)$.

\subsection{Noteworthy Sources}

The highest redshift reported in 2LAC for an HSP-BL Lac was 0.7. The 3LAC lists seven (six in the Clean Sample) HSPBL Lacs with redshifts greater than 1, six (five in the Clean Sample) of which were included in 2LAC but with other classifications or redshifts. They are briefly discussed below.

$3 F G L \mathrm{~J} 0008.0+4713$ is associated with MG4 J000800+4712. The redshift reported in 2LAC was 0.28 and its SED classification was LSP. Shaw et al. (2013) derived a redshift of 2.1 from the clear onset of the Ly $\alpha$ forest and their new procedure for estimating SED class together with WISE data classified this source as an HSP.

$3 F G L$ J0630.9-2406 is associated with TXS 0628-240, an HSP-BL Lac for which $z \gtrsim 1.238$ was determined from certain absorption features by Landt (2012). 
Table 5

Non-blazar Objects and Misaligned AGNs

\begin{tabular}{|c|c|c|c|c|c|c|}
\hline Name & 3FGL & 2FGL & 1FGL & Type & Photon Index & Notes \\
\hline NGC 1218 & $\mathrm{~J} 0308.6+0408^{\mathrm{a}}$ & $\ldots$ & $\mathrm{J} 0308.3+0403^{\mathrm{a}}$ & FRI & $2.07 \pm 0.11$ & $\ldots$ \\
\hline IC 310 & $\mathrm{~J} 0316.6+4119^{\mathrm{a}}$ & J0316.6+4119 & $\ldots$ & FRI/BLL & $1.90 \pm 0.14$ & Neronov et al. (2010) \\
\hline NGC 1275 & $\mathrm{~J} 0319.8+4130^{\mathrm{a}}$ & $\mathrm{J} 0319.8+4130^{\mathrm{a}}$ & $\mathrm{J} 0319.7+4130^{\mathrm{a}}$ & FRI & $2.07 \pm 0.01$ & Abdo et al. (2009c); Kataoka et al. (2010) \\
\hline $1 \mathrm{H} 0323+342$ & $\mathrm{~J} 0325.2+3410^{\mathrm{a}}$ & $\mathrm{J} 0324.8+3408^{\mathrm{a}}$ & $\mathrm{J} 0325.0+3403^{\mathrm{a}}$ & NLSy1 & $2.44 \pm 0.12$ & $\ldots$ \\
\hline $4 C+39.12$ & $\mathrm{~J} 0334.2+3915^{\mathrm{a}}$ & $\ldots$ & $\ldots$ & FRI/BLL? & $2.11 \pm 0.17$ & Giovannini et al. (2001) \\
\hline TXS 0348+013 & $\mathrm{J} 0351.1+0128^{\mathrm{a}}$ & $\ldots$ & $\ldots$ & SSRQ & $2.43 \pm 0.18$ & $\ldots$ \\
\hline $3 \mathrm{C} 111$ & $\mathrm{~J} 0418.5+3813$ & $\ldots$ & $\mathrm{J} 0419.0+3811$ & FRII & $2.79 \pm 0.08$ & Abdo et al. (2010e); Kataoka et al. (2011); Grandi et al. (2012) \\
\hline Pictor A & $\mathrm{J} 0519.2-4542^{\mathrm{a}}$ & $\ldots$ & $\ldots$ & FRII & $2.49 \pm 0.18$ & Brown \& Adams (2012); Kataoka et al. (2011) \\
\hline PKS 0625-35 & J0627.0-3529 & $\mathrm{J} 0627.1-3528^{\mathrm{a}}$ & $\mathrm{J} 0627.3-3530^{\mathrm{a}}$ & FRI/BLL & $1.87 \pm 0.06$ & $\ldots$ \\
\hline $4 C+52.17$ & $\mathrm{~J} 0733.5+5153$ & $\ldots$ & $\ldots$ & AGN & $1.74 \pm 0.16$ & Part of a duplicate association. Most probable counterpart is a BCU III. \\
\hline NGC 2484 & $\mathrm{~J} 0758.7+3747^{\mathrm{a}}$ & $\ldots$ & $\ldots$ & FRI & $2.16 \pm 0.16$ & quasar SDSS J075825.87+374628.7 is $0 .{ }^{\prime} 8$ away \\
\hline $4 C+39.23 B$ & J0824.9+3916 & $\ldots$ & $\ldots$ & CSS & $2.44 \pm 0.10$ & $\ldots$ \\
\hline $3 \mathrm{C} 207$ & $\mathrm{~J} 0840.8+1315^{\mathrm{a}}$ & $\mathrm{J} 0840.7+1310$ & J0840.8+1310 & SSRQ & $2.47 \pm 0.09$ & $\ldots$ \\
\hline SBS $0846+513$ & $\mathrm{~J} 0849.9+5108^{\mathrm{a}}$ & $\ldots$ & $\ldots$ & NLSy1 & $2.28 \pm 0.04$ & $\ldots$ \\
\hline 3C 221 & J0934.1+3933 & $\ldots$ & $\ldots$ & SSRQ & $2.28 \pm 0.12$ & $\ldots$ \\
\hline PMN J0948+0022 & $\mathrm{J} 0948.8+0021^{\mathrm{a}}$ & $\mathrm{J} 0948.8+0020^{\mathrm{a}}$ & $\mathrm{J} 0949.0+0021^{\mathrm{a}}$ & NLSy1 & $2.32 \pm 0.05$ & $\ldots$ \\
\hline PMN J1118-0413 & $\mathrm{J} 1118.2-0411^{\mathrm{a}}$ & $\ldots$ & $\ldots$ & AGN & $2.56 \pm 0.08$ & $\ldots$ \\
\hline B2 $1126+37$ & $\mathrm{~J} 1129.0+3705$ & $\ldots$ & $\ldots$ & AGN & $2.08 \pm 0.13$ & Part of a duplicate association. Most probable counterpart is a BLL. \\
\hline $3 \mathrm{C} 264$ & $\mathrm{~J} 1145.1+1935^{\mathrm{a}}$ & $\ldots$ & $\ldots$ & FRI & $1.98 \pm 0.20$ & $\ldots$ \\
\hline PKS $1203+04$ & $\mathrm{~J} 1205.4+0412$ & $\ldots$ & $\ldots$ & SSRQ & $2.64 \pm 0.16$ & Part of a duplicate association. The other counterpart is an FSRQ. \\
\hline M 87 & $\mathrm{~J} 1230.9+1224^{\mathrm{a}}$ & $\mathrm{J} 1230.8+1224^{\mathrm{a}}$ & $\mathrm{J} 1230.8+1223^{\mathrm{a}}$ & FRI & $2.04 \pm 0.07$ & Abdo et al. $(2009 \mathrm{~d})$ \\
\hline 3C 275.1 & $\mathrm{~J} 1244.1+1615$ & $\cdots$ & $\ldots$ & SSRQ & $2.43 \pm 0.17$ & $\ldots$ \\
\hline GB $1310+487$ & $\mathrm{~J} 1312.7+4828^{\mathrm{a}}$ & $\mathrm{J} 1312.8+4828^{\mathrm{a}}$ & $\mathrm{J} 1312.4+4827^{\mathrm{a}}$ & AGN & $2.04 \pm 0.03$ & $\ldots$ \\
\hline Cen A Core & $\mathrm{J} 1325.4-4301^{\mathrm{a}}$ & $\mathrm{J} 1325.6-4300$ & $\mathrm{~J} 1325.6-4300$ & FRI & $2.70 \pm 0.03$ & radio core \\
\hline Cen A Lobes & $\mathrm{J} 1324.0-4330 \mathrm{e}$ & $\mathrm{J} 1324.0-4330 \mathrm{e}$ & $\mathrm{J} 1322.0-4515$ & FRI & $2.53 \pm 0.05$ & giant lobes detected (Abdo et al. 2010b) \\
\hline $3 \mathrm{C} 286$ & $\mathrm{~J} 1330.5+3023^{\mathrm{a}}$ & $\ldots$ & $\ldots$ & $\mathrm{SSRQ} / \mathrm{CSS}$ & $2.60 \pm 0.16$ & $\ldots$ \\
\hline Cen B & J1346.6-6027 & J1346.6-6027 & $\ldots$ & FRI & $2.32 \pm 0.01$ & Katsuta et al. (2013) \\
\hline Circinus & $\mathrm{J} 1413.2-6518$ & $\ldots$ & $\ldots$ & Seyfert & $2.43 \pm 0.10$ & Hayashida et al. (2013) \\
\hline $3 \mathrm{C} 303$ & $\mathrm{~J} 1442.6+5156^{\mathrm{a}}$ & $\ldots$ & $\ldots$ & FRII & $1.92 \pm 0.18$ & $\ldots$ \\
\hline PKS $1502+036$ & $\mathrm{~J} 1505.1+0326^{\mathrm{a}}$ & $\mathrm{J} 1505.1+0324^{\mathrm{a}}$ & $\mathrm{J} 1505.0+0328^{\mathrm{a}}$ & NLSy1 & $2.61 \pm 0.05$ & $\ldots$ \\
\hline TXS 1613-251 & J1617.3-2519 & $\mathrm{J} 1617.6-2526 \mathrm{c}$ & $\ldots$ & AGN & $2.59 \pm 0.10$ & Part of a duplicate association. Most probable counterpart is a BCU II. \\
\hline PKS 1617-235 & $\mathrm{J} 1621.1-2331^{\mathrm{a}}$ & $\mathrm{J} 1620.5-2320 \mathrm{c}$ & $\ldots$ & AGN & $2.50 \pm 0.23$ & $\ldots$ \\
\hline NGC 6251 & $\mathrm{~J} 1630.6+8232^{\mathrm{a}}$ & $\mathrm{J} 1629.4+8236$ & $\mathrm{~J} 1635.4+8228^{\mathrm{a}}$ & FRI & $2.22 \pm 0.08$ & $\ldots$ \\
\hline $3 \mathrm{C} 380$ & $\mathrm{~J} 1829.6+4844^{\mathrm{a}}$ & $\mathrm{J} 1829.7+4846^{\mathrm{a}}$ & $\mathrm{J} 1829.8+4845^{\mathrm{a}}$ & $\mathrm{SSRQ} / \mathrm{CSS}$ & $2.37 \pm 0.04$ & $\ldots$ \\
\hline PKS 2004-447 & $\mathrm{J} 2007.8-4429^{\mathrm{a}}$ & $\mathrm{J} 2007.9-4430^{\mathrm{a}}$ & $\mathrm{J} 2007.9-4430^{\mathrm{a}}$ & NLSy1 & $2.47 \pm 0.09$ & $\ldots$ \\
\hline
\end{tabular}

Notes. SSRQ implies FRII. The table includes the 34 non-blazar objects and MAGNs at all latitudes associated with 3FGL sources (Cen A Core and Cen A Lobes constitute a single object).

${ }^{a}$ Refers to sources included in the Clean Sample of a given catalog. 


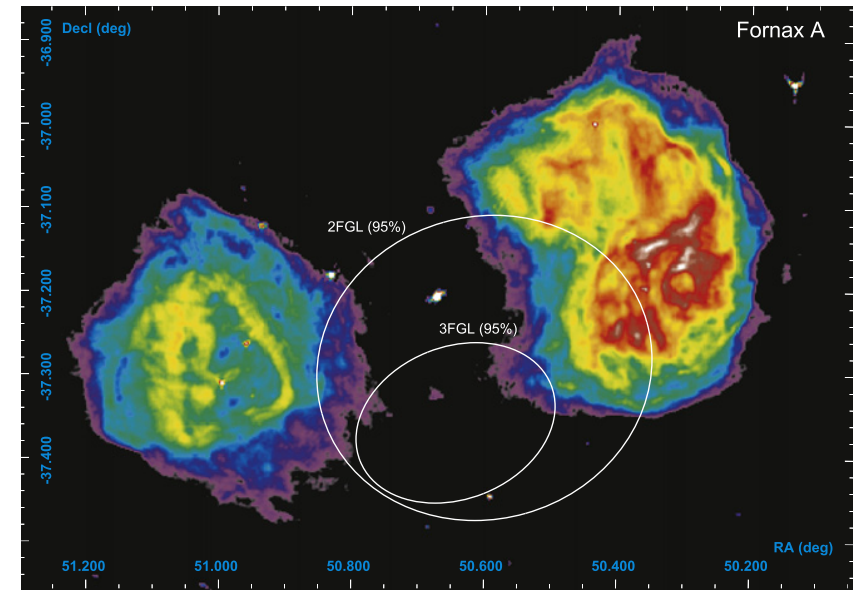

Figure 6. VLA image of Fornax A at $1.5 \mathrm{GHz}$ (Fomalont et al. 1989). The 95\% error ellipses of the closest 3FGL and 2FGL sources are overlaid.

$3 F G L J 1109.4+2411$ is associated with $1 \mathrm{ES} 1106+244$ and new spectroscopy from SDSS changed the redshift to 1.220.

$3 F G L$ J1312.5-2155 is associated with PKS 1309-216. In Shaw et al. (2013) a plausible $\mathrm{Mg}$ II feature is found; this single-line identification is in a small allowed redshift range $(z \simeq 1.6)$. However, previous data (Massaro et al. 2009) show a questionable redshift of 1.491 .

$3 F G L$ J2116.1+3339 is associated with B2 2114+33. The redshift quoted in $2 \mathrm{LAC}$ was 0.35 , but a recent measurement by Shaw et al. (2013) gives $z=1.596$, identifying a significant broad emission feature with $\mathrm{C}$ IV, consistent with a weak bump in the far blue at Ly $\alpha$. A lower redshift is possible if the purported Ly $\alpha$ line is not real.

The newly detected source is 3FGL J0814.5+2943, associated with FBQS J081421.2+294021 at $z=1.084$ (from SDSS DR3, Ahn et al. 2012).

The highest redshift BL Lac object is $3 F G L J 1450.9+5200$, associated with BZB J1450+5201 with redshift $z=2.41$ coming from new observations in Shaw et al. (2013). The presence of the Ly $\alpha$ forest can suppress a part of the optical spectrum, resulting in ISP classification, so the intrinsic synchrotron peak position is probably greater than our estimate.

A low-redshift source, reported as a BCU in BZCAT, has recently been classified as an FSRQ by G. Chiaro \& D. Bastieri (2014, private communication): SBS 1646+499 (3FGL J1647.4+4950) with $z=0.0467$.

Two HSP-FSRQs have been detected: BZB J0202+0849 (3FGL J0202.3+0851) and NVSS J025037+171209 (3FGL J0250.6+1713) with LAT spectral photon indices of $2.05 \pm$ 0.16 and $1.98 \pm 0.19$, respectively. 3FGL J0202.3+0851 was classified as a BL Lac in 1LAC but new observations from Shaw et al. (2013) led to a reclassification as an FSRQ. These objects are probably transitional objects that show broad lines in the optical band when the continuum is low (see, e.g., Ruan et al. 2014).

\subsection{Low Galactic Latitude AGNs}

Because of the intrinsic incompleteness of the counterpart catalogs in this sky area $\left(|b|<10^{\circ}\right)$, these sources are treated separately and are not included in the 3LAC or in the analyses presented in the rest of the paper. We report associations for 182 blazars $\left(75 \%\right.$ more than in 2LAC) located at $|b|<10^{\circ}: 24$
FSRQs, 30 BL Lacs, 125 BCUs, and 3 non-blazar AGNs. They are listed in Table 6. Extrapolating from the number of highlatitude sources and assuming the same sensitivity, about 340 sources would be expected in this area. The discrepancy between expected and actual source numbers stems from the dual effect of a higher detection threshold due to a higher Galactic diffuse emission background (see Figure 1) and a higher incompleteness of the counterpart catalogs for this area.

\subsection{Comparison with $1 L A C$ and $2 L A C$}

The revised 2LAC sample (Ackermann et al. 2015) includes 929 sources, 65 of which are missing in 3LAC (Table 7). Most do not make the TS cut over the 4 year-long period, probably mainly due to variability. On the other hand, 56 unassociated sources in the 2FGL are now associated with blazars, thanks to a more complete set of counterpart catalogs and more precise localizations for the gamma-ray sources (arising from greater statistics and an improved instrument point-spread function). A total of 27 1FGL sources (not necessarily all in 1LAC) that were not listed in 2LAC are now included in 3LAC. Some 51 2LAC sources have changed classifications in 3LAC, mostly due to improved data: 8 AGNs into BCUs, 1 AGN into a BL Lac, 39 BCUs into 34 BL Lacs and 5 FSRQs, one FSRQ into a BL Lac (TXS 0404+075) and two BL Lacs into FSRQs (B2 1040+24A and 4C+15.54).

\subsection{Flaring Sources Detected in the Flare Advocate Service}

The 3LAC catalog lists sources detected with high significance during 48 months of observation. Some blazars flare during a limited time only and may be missing in 3LAC. If bright enough, some of them are caught in near-real time by the Fermi Flare Advocate service, also known as Gamma-ray Sky Watcher (FA-GSW), which we briefly describe here.

A day-by-day review of the whole gamma-ray sky, both by a human-in-the-loop and by automated science processing analysis (see, e.g., Chiang et al. 2012), results in the calculation of preliminary source fluxes, tentative localizations, and counterpart associations for any significant source detection. This service serves as an important resource for the scientific community by providing alerts on flaring or transient sources and by producing seeds for follow-up variability and multiwavelength $^{80}$ studies (see, e.g., Ciprini \& Thompson 2013).

Since the beginning of the mission, daily reports are compiled internally to the Collaboration, while information and news are communicated via the LAT-MW mailing-list, ${ }^{81}$ published in The Astronomer's Telegrams (ATels, ${ }^{82}$ special GCN notices, ${ }^{83}$ and weekly summaries in the Fermi Sky Blog. ${ }^{84}$ A total of 201 ATels were posted on behalf of the LAT Collaboration in the 48 month period considered in the 3FGL/ 3LAC, specifically from 2008 July 24 (the first ATel\#1628) to 2012 July 29 (ATel\#4285), primarily derived from the FAGSW service. Some 143 ATels contained alerts and preliminary results about blazars and other AGN targets ${ }^{85}$ referring to 71 different FSRQs, 18 different BL Lac objects,

\footnotetext{
${ }^{80}$ For example the LAT MW Coordinating Group page: confluence.slac. stanford.edu/X/YQw

81 Address: lists.nasa.gov/mailman/listinfo/gammamw/

82 www-glast.stanford.edu/cgi-bin/pub_rapid

83 gcn.gsfc.nasa.gov/gcn/fermi_lat_mon_trans.html

84 The Fermi Gamma-ray Sky Blog: fermisky.blogspot.com

85 An interactive incremental list is available at: www.asdc.asi.it/feratel/.
} 
Table 6

Low-latitude $\left(|b|<10^{\circ}\right)$ Sample

\begin{tabular}{|c|c|c|c|c|c|c|c|c|c|c|c|c|c|}
\hline $\begin{array}{l}\text { 3FGL Source } \\
\text { Name } \\
(1)\end{array}$ & $\begin{array}{l}\text { Counterpart } \\
\text { Name } \\
\text { (2) }\end{array}$ & $\begin{array}{l}\text { R.A. } \\
\left({ }^{\circ}\right) \\
(3)\end{array}$ & $\begin{array}{l}\text { Decl. } \\
\left({ }^{\circ}\right) \\
(4)\end{array}$ & $\begin{array}{c}\text { AngSep } \\
\left({ }^{\circ}\right) \\
(5)\end{array}$ & $\begin{array}{l}\theta_{95} \\
\left({ }^{\circ}\right) \\
(6)\end{array}$ & $\begin{array}{l}\text { Optical } \\
\text { Class } \\
(7)\end{array}$ & $\begin{array}{l}\text { SED } \\
\text { Class } \\
(8)\end{array}$ & $\begin{array}{c}\log \left(\nu_{\text {peak }}^{\text {S,meas }}\right) \\
(9)\end{array}$ & $\begin{array}{c}\log \left(\nu_{\text {peak }}^{\mathrm{S}}\right) \\
(10)\end{array}$ & (11) & $\begin{array}{l}\text { Prob. } \\
\text { Bay. } \\
(12)\end{array}$ & $\begin{array}{c}\text { Rel. } \\
\mathrm{LR}_{\mathrm{RG}} \\
(13)\end{array}$ & $\begin{array}{c}\text { Rel. } \\
\mathrm{LR}_{\mathrm{XG}} \\
(14)\end{array}$ \\
\hline J0012.4+7040 & TXS $0008+704$ & 2.88293 & 70.75878 & 0.115 & 0.105 & BCU II & LSP & 13.075 & 13.075 & $\ldots$ & 0.912 & 0.856 & $\ldots$ \\
\hline J0014.6+6119 & $4 C+60.01$ & 3.70330 & 61.29543 & 0.031 & 0.061 & BCU II & LSP & 13.113 & 13.113 & $\ldots$ & 0.997 & 0.976 & \\
\hline$J 0014.7+5802$ & 1RXS J001442.2+580201 & 3.67471 & 58.03404 & 0.009 & 0.055 & BLL & HSP & 16.640 & 16.640 & $\ldots$ & $\ldots$ & $\ldots$ & 0.936 \\
\hline J0015.7+5552 & GB6 J0015+5551 & 3.91737 & 55.86226 & 0.018 & 0.043 & BCU II & HSP & 15.791 & 15.791 & $\cdots$ & 0.998 & 0.868 & 0.952 \\
\hline J0035.9+5949 & 1ES $0033+595$ & 8.96935 & 59.83459 & 0.010 & 0.018 & BLL & HSP & 17.120 & 17.120 & $\ldots$ & 1.000 & 0.935 & 0.979 \\
\hline J0047.0+5658 & GB6 J0047+5657 & 11.75179 & 56.96178 & 0.013 & 0.040 & BLL & $\ldots$ & $\ldots$ & $\ldots$ & 0.74700 & 1.000 & 0.910 & $\ldots$ \\
\hline J0047.9+5447 & 1RXS J004754.5+544758 & 11.96611 & 54.79579 & 0.010 & 0.060 & BCU II & HSP & 15.896 & 15.896 & & $\ldots$ & $\ldots$ & 0.890 \\
\hline J0102.8+5825 & TXS $0059+581$ & 15.69068 & 58.40309 & 0.023 & 0.020 & FSRQ & LSP & 12.725 & 12.941 & 0.64400 & 0.999 & 0.956 & $\ldots$ \\
\hline J0103.4+5336 & 1RXS J010325.9+533721 & 15.85816 & 53.62036 & 0.006 & 0.042 & BLL & $\ldots$ & $\ldots$ & $\ldots$ & $\ldots$ & $\ldots$ & 0.824 & 0.934 \\
\hline J0109.8+6132 & TXS 0106+612 & 17.44310 & 61.55846 & 0.015 & 0.033 & FSRQ & LSP & 13.290 & 13.541 & 0.78300 & 0.999 & 0.934 & $\ldots$ \\
\hline J0110.2+6806 & $4 C+67.04$ & 17.55364 & 68.09478 & 0.022 & 0.023 & BLL & ISP & 14.864 & 14.864 & $\ldots$ & 1.000 & 0.972 & 0.895 \\
\hline J0131.2+6120 & 1RXS J013106.4+612035 & 22.78011 & 61.34260 & 0.013 & 0.022 & BLL & HSP & 16.300 & 16.300 & $\ldots$ & 0.999 & 0.850 & 0.979 \\
\hline J0131.3+5548 & TXS $0128+554$ & 22.80760 & 55.75361 & 0.056 & 0.082 & $\mathrm{BCU} \mathrm{I}$ & $\ldots$ & $\ldots$ & $\ldots$ & 0.03649 & 0.986 & 0.828 & 0.806 \\
\hline J0135.0+6927 & TXS 0130+691 & 23.66984 & 69.41969 & 0.055 & 0.095 & BCU III & $\ldots$ & $\ldots$ & $\ldots$ & $\ldots$ & 0.984 & 0.830 & $\ldots$ \\
\hline J0137.8+5813 & 1RXS J013748.0+581422 & 24.46032 & 58.23648 & 0.005 & 0.032 & BCU II & HSP & 16.580 & 16.580 & $\cdots$ & 0.999 & 0.921 & 0.969 \\
\hline J0148.3+5200 & GB6 J0148+5202 & 27.08473 & 52.03470 & 0.025 & 0.039 & BCU III & $\ldots$ & $\ldots$ & $\ldots$ & $\ldots$ & 0.996 & $\ldots$ & $\ldots$ \\
\hline J0153.4+7114 & TXS $0149+710$ & 28.35771 & 71.25180 & 0.010 & 0.037 & $\mathrm{BCU} \mathrm{I}$ & HSP & 15.690 & 15.699 & 0.02200 & 1.000 & 0.953 & 0.930 \\
\hline $\mathrm{J} 0211.7+5402$ & TXS $0207+538$ & 32.73495 & 54.08692 & 0.120 & 0.128 & BCU III & $\ldots$ & $\ldots$ & $\ldots$ & $\ldots$ & $\ldots$ & 0.827 & . \\
\hline $\mathrm{J} 0214.4+5143$ & TXS $0210+515$ & 33.57473 & 51.74776 & 0.034 & 0.044 & BLL & HSP & 15.020 & 15.041 & 0.04900 & 0.999 & 0.905 & 0.944 \\
\hline J0217.3+6209 & TXS $0213+619$ & 34.26049 & 62.19274 & 0.056 & 0.102 & BCU III & $\ldots$ & $\ldots$ & $\ldots$ & $\ldots$ & $\ldots$ & 0.800 & $\ldots$ \\
\hline J0223.3+6820 & NVSS J022304+682154 & 35.76891 & 68.36528 & 0.031 & 0.037 & BCU II & HSP & 15.800 & 15.800 & $\ldots$ & 0.991 & $\ldots$ & $\cdots$ \\
\hline $\mathrm{J} 0223.5+6313$ & TXS 0219+628 & 35.87363 & 63.12177 & 0.104 & 0.152 & BCU III & $\ldots$ & $\ldots$ & $\ldots$ & $\cdots$ & 0.945 & $\ldots$ & $\ldots$ \\
\hline J0228.5+6703 & GB6 J0229+6706 & 37.34410 & 67.11042 & 0.099 & 0.166 & BCU III & $\ldots$ & $\ldots$ & $\ldots$ & $\ldots$ & 0.953 & $\ldots$ & \\
\hline J0241.3+6542 & TXS $0237+655$ & 40.34061 & 65.71988 & 0.018 & 0.043 & BCU II & HSP & 15.500 & 15.500 & $\ldots$ & $\ldots$ & 0.903 & 0.910 \\
\hline J0250.6+5630 & NVSS J025047+562935 & 42.69830 & 56.49317 & 0.030 & 0.052 & BCU II & HSP & 16.138 & 16.138 & $\ldots$ & $\ldots$ & $\ldots$ & 0.890 \\
\hline J0253.8+5104 & NVSS J025357+510256 & 43.49003 & 51.04902 & 0.022 & 0.075 & FSRQ & LSP & 12.500 & 12.936 & 1.73200 & 1.000 & 0.912 & $\ldots$ \\
\hline J0302.0+5335 & GB6 J0302+5331 & 45.59473 & 53.52958 & 0.081 & 0.076 & BCU II & HSP & 15.988 & 15.988 & $\ldots$ & 0.965 & $\ldots$ & $\ldots$ \\
\hline $\mathrm{J} 0303.6+4716$ & $4 C+47.08$ & 45.89684 & 47.27119 & 0.017 & 0.031 & BLL & ISP & 14.000 & 14.000 & $\ldots$ & 1.000 & 0.965 & $\ldots$ \\
\hline J0304.9+6817 & TXS 0259+681 & 46.09168 & 68.36041 & 0.082 & 0.076 & BCU II & LSP & 12.725 & 12.725 & $\ldots$ & 0.911 & 0.920 & $\cdots$ \\
\hline $\mathrm{J} 0332.0+6308$ & GB6 J0331+6307 & 52.97465 & 63.13727 & 0.016 & 0.051 & BCU II & ISP & 14.150 & 14.150 & $\ldots$ & 0.998 & 0.816 & $\ldots$ \\
\hline J0333.9+6538 & TXS 0329+654 & 53.48641 & 65.61561 & 0.022 & 0.034 & BLL & HSP & 15.200 & 15.200 & $\ldots$ & 0.998 & 0.924 & 0.885 \\
\hline J0352.9+5655 & GB6 J0353+5654 & 58.28989 & 56.90859 & 0.032 & 0.046 & BCU II & HSP & 16.315 & 16.315 & $\cdots$ & 0.996 & 0.820 & $\ldots$ \\
\hline J0354.1+4643 & B3 $0350+465$ & 58.62505 & 46.72188 & 0.065 & 0.118 & BCU III & $\ldots$ & & $\ldots$ & $\ldots$ & 0.977 & 0.904 & $\ldots$ \\
\hline $\mathrm{J} 0358.8+6002$ & TXS 0354+599 & 59.76100 & 60.08946 & 0.055 & 0.121 & FSRQ & LSP & 12.905 & 13.068 & 0.45500 & 0.993 & 0.919 & 0.830 \\
\hline $\mathrm{J} 0418.5+3813$ & 3C 111 & 64.58866 & 38.02661 & 0.198 & 0.168 & RDG & $\ldots$ & $\ldots$ & $\ldots$ & 0.04850 & 0.961 & 0.949 & 0.807 \\
\hline J0423.8+4150 & $4 \mathrm{C}+41.11$ & 65.98337 & 41.83409 & 0.012 & 0.021 & BLL & LSP & 13.180 & 13.180 & & 1.000 & 0.980 & \\
\hline J0425.2+6319 & 1RXS J042523.0+632016 & 66.35324 & 63.33486 & 0.019 & 0.040 & BCU II & HSP & 16.050 & 16.050 & $\cdots$ & $\ldots$ & 0.804 & 0.920 \\
\hline $\mathrm{J} 0444.5+3425$ & B2 $0441+34$ & 71.15083 & 34.42877 & 0.014 & 0.074 & BCU II & LSP & 13.005 & 13.005 & $\cdots$ & 0.997 & 0.880 & $\ldots$ \\
\hline J0501.8+3046 & 1RXS J050140.8+304831 & 75.42145 & 30.80727 & 0.051 & 0.059 & BCU II & HSP & 16.100 & 16.100 & $\ldots$ & $\ldots$ & $\ldots$ & 0.886 \\
\hline J0502.7+3438 & MG2 J050234+3436 & 75.62478 & 34.60960 & 0.064 & 0.078 & BCU III & $\ldots$ & $\ldots$ & $\ldots$ & $\cdots$ & 0.983 & 0.820 & $\ldots$ \\
\hline J0503.4+4522 & 1RXS J050339.8+451715 & 75.91491 & 45.28320 & 0.098 & 0.086 & BCU II & HSP & 15.645 & 15.645 & $\cdots$ & $\ldots$ & $\ldots$ & 0.844 \\
\hline J0512.2+2918 & B2 $0509+29$ & 78.17586 & 29.45100 & 0.170 & 0.527 & BCU III & $\ldots$ & $\ldots$ & $\ldots$ & $\cdots$ & 0.891 & $\ldots$ & $\ldots$ \\
\hline $\mathrm{J} 0512.9+4038$ & B3 $0509+406$ & 78.21893 & 40.69545 & 0.054 & 0.073 & BCU II & LSP & 13.635 & 13.635 & $\ldots$ & 0.999 & 0.933 & $\cdots$ \\
\hline J0517.4+4540 & $4 C+45.08$ & 79.37041 & 45.61802 & 0.050 & 0.154 & FSRQ & LSP & 12.900 & 13.165 & 0.83900 & 0.990 & 0.907 & $\cdots$ \\
\hline J0519.3+2746 & $4 \mathrm{C}+27.15$ & 79.88761 & 27.73454 & 0.051 & 0.110 & BCU III & $\cdots$ & $\ldots$ & $\ldots$ & $\ldots$ & 0.992 & 0.944 & $\cdots$ \\
\hline $\mathrm{J} 0521.7+2113$ & TXS $0518+211$ & 80.44152 & 21.21429 & 0.008 & 0.014 & BLL & ISP & 14.335 & 14.380 & 0.10800 & 1.000 & 0.969 & 0.961 \\
\hline $\mathrm{J} 0526.0+4253$ & NVSS J052520+425520 & 81.33690 & 42.92225 & 0.140 & 0.150 & BCU II & LSP & 13.145 & 13.145 & $\ldots$ & 0.942 & $\ldots$ & . \\
\hline $\mathrm{J} 0528.3+1815$ & 1RXS J052829.6+181657 & 82.12341 & 18.28188 & 0.048 & 0.060 & BCU III & $\ldots$ & $\ldots$ & $\ldots$ & $\ldots$ & $\ldots$ & $\ldots$ & 0.929 \\
\hline J0533.2+4822 & TXS $0529+483$ & 83.31611 & 48.38134 & 0.007 & 0.031 & FSRQ & LSP & 13.040 & 13.375 & 1.16200 & 1.000 & 0.950 & 0.876 \\
\hline
\end{tabular}


Table 6

(Continued)

\begin{tabular}{|c|c|c|c|c|c|c|c|c|c|c|c|c|c|}
\hline $\begin{array}{l}\text { 3FGL Source } \\
\text { Name } \\
(1)\end{array}$ & $\begin{array}{l}\text { Counterpart } \\
\text { Name } \\
(2)\end{array}$ & $\begin{array}{l}\text { R.A. } \\
\left({ }^{\circ}\right) \\
(3) \\
\end{array}$ & $\begin{array}{c}\text { Decl. } \\
\left({ }^{\circ}\right) \\
(4) \\
\end{array}$ & $\begin{array}{c}\text { AngSep } \\
\left({ }^{\circ}\right) \\
(5) \\
\end{array}$ & $\begin{array}{l}\theta_{95} \\
\left({ }^{\circ}\right) \\
(6)\end{array}$ & $\begin{array}{l}\text { Optical } \\
\text { Class } \\
(7) \\
\end{array}$ & $\begin{array}{c}\text { SED } \\
\text { Class } \\
(8) \\
\end{array}$ & $\begin{array}{c}\log \left(\nu_{\text {peak }}^{\text {S,meas }}\right) \\
(9)\end{array}$ & $\begin{array}{c}\log \left(\nu_{\text {peak }}^{\mathrm{S}}\right) \\
(10)\end{array}$ & $\begin{array}{c}z \\
(11)\end{array}$ & $\begin{array}{l}\text { Prob. } \\
\text { Bay. } \\
(12)\end{array}$ & $\begin{array}{c}\text { Rel. } \\
\text { LR }_{\text {RG }} \\
(13)\end{array}$ & $\begin{array}{c}\text { Rel. } \\
\mathrm{LR}_{\mathrm{XG}} \\
(14)\end{array}$ \\
\hline $\mathrm{J} 0539.8+1434$ & TXS $0536+145$ & 84.92652 & 14.56266 & 0.031 & 0.071 & FSRQ & LSP & 12.445 & 13.012 & 2.69000 & 0.999 & 0.911 & $\cdots$ \\
\hline J0601.0+3837 & B2 $0557+38$ & 90.26196 & 38.64144 & 0.017 & 0.053 & BLL & LSP & 13.810 & 13.810 & $\ldots$ & $\ldots$ & 0.945 & $\cdots$ \\
\hline $\mathrm{J} 0603.8+2155$ & $4 \mathrm{C}+22.12$ & 90.96482 & 21.99381 & 0.066 & 0.058 & BCU II & LSP & 13.250 & 13.250 & $\ldots$ & 0.981 & 0.955 & $\cdots$ \\
\hline J0611.7+2759 & GB6 J0611+2803 & 92.93284 & 28.06449 & 0.067 & 0.107 & BCU III & $\ldots$ & $\ldots$ & $\ldots$ & $\cdots$ & 0.991 & $\cdots$ & $\ldots$ \\
\hline J0620.4+2644 & RX J0620.6+2644 & 95.16716 & 26.72524 & 0.044 & 0.063 & BCU II & HSP & 16.085 & 16.085 & $\cdots$ & $\cdots$ & 0.805 & 0.940 \\
\hline J0622.9+3326 & B2 0619+33 & 95.71759 & 33.43622 & 0.014 & 0.018 & BCU II & ISP & 14.050 & 14.050 & $\ldots$ & 0.999 & 0.938 & $\ldots$ \\
\hline J0623.3+3043 & GB6 J0623+3045 & 95.81747 & 30.74889 & 0.025 & 0.065 & BCU II & ISP & 14.790 & 14.790 & $\ldots$ & 0.996 & 0.800 & $\ldots$ \\
\hline J0631.2+2019 & TXS 0628+203 & 97.75443 & 20.34978 & 0.050 & 0.106 & BCU II & HSP & 15.000 & 15.000 & $\ldots$ & 0.990 & 0.862 & \\
\hline J0640.0-1252 & TXS 0637-128 & 100.02993 & -12.88761 & 0.015 & 0.040 & BCU II & HSP & 16.050 & 16.050 & $\cdots$ & 0.998 & 0.915 & 0.967 \\
\hline J0641.8-0319 & TXS 0639-032 & 100.46305 & -3.34683 & 0.029 & 0.142 & BCU II & LSP & 12.760 & 12.760 & $\ldots$ & 0.987 & 0.920 & $\ldots$ \\
\hline $\mathrm{J} 0643.2+0859$ & PMN J0643+0857 & 100.86019 & 8.96056 & 0.066 & 0.063 & FSRQ & LSP & 13.000 & 13.275 & 0.88200 & 0.975 & $\ldots$ & $\ldots$ \\
\hline J0648.1+1606 & 1RXS J064814.1+160708 & 102.05790 & 16.11576 & 0.018 & 0.045 & BCU II & HSP & 16.300 & 16.300 & & $\cdots$ & $\ldots$ & 0.904 \\
\hline J0648.8+1516 & RX J0648.7+1516 & 102.19854 & 15.27355 & 0.007 & 0.029 & BLL & HSP & 15.850 & 15.922 & 0.17900 & 1.000 & 0.892 & 0.976 \\
\hline J0648.8-1740 & TXS 0646-176 & 102.11874 & -17.73484 & 0.109 & 0.155 & FSRQ & LSP & 12.480 & 12.829 & 1.23200 & 0.995 & 0.898 & $\cdots$ \\
\hline J0650.4-1636 & PKS 0648-16 & 102.60242 & -16.62770 & 0.019 & 0.094 & BCU II & LSP & 11.465 & 11.465 & $\cdots$ & 0.998 & 0.954 & $\cdots$ \\
\hline J0650.5+2055 & 1RXS J065033.9+205603 & 102.64681 & 20.93242 & 0.003 & 0.040 & BCU II & HSP & 15.650 & 15.650 & $\ldots$ & $\cdots$ & $\cdots$ & 0.892 \\
\hline J0654.5+0926 & RX J0654.3+0925 & 103.61306 & 9.42644 & 0.032 & 0.231 & BCU II & HSP & 15.350 & 15.350 & $\ldots$ & $\ldots$ & $\ldots$ & 0.840 \\
\hline J0656.2-0323 & TXS 0653-033 & 104.04634 & -3.38522 & 0.009 & 0.053 & FSRQ & LSP & 13.495 & 13.708 & 0.63400 & 1.000 & 0.929 & $\ldots$ \\
\hline J0658.6+0636 & NVSS J065844+063711 & 104.68735 & 6.61943 & 0.039 & 0.068 & BCU II & HSP & 15.000 & 15.000 & $\ldots$ & 0.999 & $\ldots$ & $\ldots$ \\
\hline J0700.0+1709 & TXS $0657+172$ & 105.00636 & 17.15603 & 0.016 & 0.116 & BCU II & LSP & 12.725 & 12.725 & $\ldots$ & 0.999 & 0.910 & $\ldots$ \\
\hline J0700.2+1304 & GB6 J0700+1304 & 105.05963 & 13.07345 & 0.013 & 0.065 & BCU II & HSP & 15.425 & 15.425 & $\ldots$ & 0.998 & $\cdots$ & $\ldots$ \\
\hline J0702.7-1952 & TXS 0700-197 & 105.67875 & -19.85612 & 0.015 & 0.053 & BLL & ISP & 14.050 & 14.050 & $\ldots$ & 0.999 & 0.937 & $\ldots$ \\
\hline J0709.7-0256 & PMN J0709-0255 & 107.43773 & -2.92153 & 0.019 & 0.039 & BLL & LSP & 12.830 & 13.223 & 1.47200 & 0.998 & 0.898 & $\cdots$ \\
\hline J0721.4+0404 & PMN J0721+0406 & 110.34963 & 4.11228 & 0.041 & 0.082 & FSRQ & LSP & 12.700 & 12.921 & 0.66500 & 0.999 & 0.881 & \\
\hline J0723.2-0728 & 1RXS J072259.5-073131 & 110.74895 & -7.52649 & 0.079 & 0.090 & BCU III & $\ldots$ & $\ldots$ & $\ldots$ & $\ldots$ & 0.976 & $\ldots$ & 0.899 \\
\hline J0725.8-0054 & PKS 0723-008 & 111.46100 & -0.91571 & 0.010 & 0.044 & BCU I & LSP & 13.355 & 13.407 & 0.12800 & 1.000 & 0.967 & $\ldots$ \\
\hline J0729.5-3127 & NVSS J072922-313128 & 112.34570 & -31.52438 & 0.078 & 0.157 & BCU II & LSP & 13.133 & 13.133 & $\ldots$ & 0.979 & $\ldots$ & $\cdots$ \\
\hline J0730.2-1141 & PKS 0727-11 & 112.57964 & -11.68683 & 0.006 & 0.013 & FSRQ & LSP & 12.300 & 12.713 & 1.58900 & 1.000 & 0.989 & $\ldots$ \\
\hline J0730.5-0537 & TXS 0728-054 & 112.61849 & -5.59636 & 0.027 & 0.050 & BCU II & HSP & 15.200 & 15.200 & $\ldots$ & 0.997 & 0.882 & $\ldots$ \\
\hline J0744.1-3804 & PMN J0743-3804 & 115.93736 & -38.06650 & 0.080 & 0.269 & BCU III & $\ldots$ & $\ldots$ & $\ldots$ & $\cdots$ & 0.936 & $\ldots$ & $\cdots$ \\
\hline J0744.8-4028 & PMN J0744-4032 & 116.15929 & -40.53806 & 0.083 & 0.078 & BCU II & LSP & 12.620 & 12.620 & $\ldots$ & 0.872 & $\ldots$ & $\ldots$ \\
\hline J0746.6-0706 & PMN J0746-0709 & 116.61456 & -7.16379 & 0.067 & 0.098 & BCU II & ISP & 14.230 & 14.230 & $\ldots$ & 0.983 & $\cdots$ & $\ldots$ \\
\hline J0747.2-3311 & PKS 0745-330 & 116.83201 & -33.17971 & 0.016 & 0.033 & BCU II & LSP & 13.850 & 13.850 & $\ldots$ & 1.000 & 0.958 & $\ldots$ \\
\hline J0748.0-1639 & TXS 0745-165 & 117.01285 & -16.66396 & 0.009 & 0.125 & BCU II & LSP & 11.920 & 11.920 & $\cdots$ & 0.997 & 0.915 & $\cdots$ \\
\hline J0754.4-1148 & TXS 0752-116 & 118.61024 & -11.78804 & 0.027 & 0.039 & BLL & LSP & 13.355 & 13.355 & $\cdots$ & 1.000 & 0.953 & $\cdots$ \\
\hline J0804.0-3629 & NVSS J080405-362919 & 121.02237 & -36.48863 & 0.008 & 0.045 & BCU II & HSP & 15.920 & 15.920 & $\ldots$ & 0.999 & 0.852 & $\ldots$ \\
\hline J0816.7-2421 & PMN J0816-2421 & 124.16838 & -24.35183 & 0.012 & 0.073 & BCU II & LSP & 12.340 & 12.340 & $\cdots$ & 0.999 & 0.873 & $\ldots$ \\
\hline J0825.8-3217 & PKS 0823-321 & 126.46405 & -32.30645 & 0.023 & 0.066 & BCU II & ISP & 14.030 & 14.030 & $\ldots$ & 0.999 & 0.914 & $\cdots$ \\
\hline J0825.9-2230 & PKS 0823-223 & 126.50655 & -22.50756 & 0.008 & 0.018 & BLL & ISP & 14.160 & 14.441 & 0.91100 & 1.000 & 0.966 & 0.947 \\
\hline J0828.8-2420 & NVSS J082841-241850 & 127.17383 & -24.31403 & 0.041 & 0.098 & BCU III & $\ldots$ & $\ldots$ & $\ldots$ & $\ldots$ & $\cdots$ & 0.853 & \\
\hline J0841.3-3554 & NVSS J084121-355506 & 130.34017 & -35.91823 & 0.014 & 0.027 & BCU II & HSP & 15.956 & 15.956 & $\cdots$ & 1.000 & 0.892 & $\cdots$ \\
\hline J0845.1-5458 & PMN J0845-5458 & 131.26034 & -54.96904 & 0.021 & 0.039 & BCU II & LSP & 13.005 & 13.005 & $\cdots$ & 1.000 & 0.981 & 0.828 \\
\hline J0849.5-2912 & NVSS J084922-291149 & 132.34210 & -29.19734 & 0.043 & 0.064 & BCU II & ISP & 14.504 & 14.504 & $\cdots$ & 0.988 & $\cdots$ & $\cdots$ \\
\hline J0849.9-3540 & PMN J0849-3541 & 132.44010 & -35.68369 & 0.034 & 0.052 & BCU II & LSP & 12.900 & 12.900 & $\cdots$ & 1.000 & 0.913 & $\cdots$ \\
\hline J0852.6-5756 & PMN J0852-5755 & 133.16136 & -57.92495 & 0.022 & 0.050 & BCU II & LSP & 13.076 & 13.076 & $\cdots$ & 0.999 & $\cdots$ & 0.858 \\
\hline J0853.0-3654 & NVSS J085310-365820 & 133.29384 & -36.97236 & 0.061 & 0.047 & BCU II & HSP & 15.660 & 15.660 & $\cdots$ & 0.883 & 0.810 & $\cdots$ \\
\hline J0858.1-3130 & 1RXS J085802.6-313043 & 134.51195 & -31.51118 & 0.029 & 0.091 & BCU II & HSP & 16.235 & 16.235 & $\cdots$ & $\cdots$ & $\cdots$ & 0.913 \\
\hline J0904.8-3516 & NVSS J090442-351423 & 136.17658 & -35.24010 & 0.053 & 0.084 & BCU II & ISP & 14.171 & 14.171 & $\ldots$ & 0.988 & 0.864 & \\
\hline
\end{tabular}


Table 6

(Continued)

\begin{tabular}{|c|c|c|c|c|c|c|c|c|c|c|c|c|c|}
\hline $\begin{array}{l}\text { 3FGL Source } \\
\text { Name } \\
\text { (1) }\end{array}$ & $\begin{array}{l}\text { Counterpart } \\
\text { Name } \\
(2)\end{array}$ & $\begin{array}{l}\text { R.A. } \\
\left({ }^{\circ}\right) \\
(3) \\
\end{array}$ & $\begin{array}{l}\text { Decl. } \\
\left({ }^{\circ}\right) \\
(4) \\
\end{array}$ & $\begin{array}{c}\text { AngSep } \\
\left({ }^{\circ}\right) \\
(5)\end{array}$ & $\begin{array}{l}\theta_{95} \\
\left({ }^{\circ}\right) \\
(6) \\
\end{array}$ & $\begin{array}{c}\text { Optical } \\
\text { Class } \\
(7)\end{array}$ & $\begin{array}{l}\text { SED } \\
\text { Class } \\
(8)\end{array}$ & $\begin{array}{c}\log \left(\nu_{\text {peak }}^{\text {S,meas }}\right) \\
(9)\end{array}$ & $\begin{array}{c}\log \left(\nu_{\text {peak }}^{\mathrm{S}}\right) \\
(10)\end{array}$ & $\begin{array}{c}z \\
(11)\end{array}$ & $\begin{array}{l}\text { Prob. } \\
\text { Bay. } \\
(12)\end{array}$ & $\begin{array}{c}\text { Rel. } \\
\mathrm{LR}_{\mathrm{RG}} \\
(13) \\
\end{array}$ & $\begin{array}{c}\text { Rel. } \\
\text { LRR }_{\mathrm{XG}} \\
(14)\end{array}$ \\
\hline J0904.8-5734 & PKS 0903-57 & 136.22158 & -57.58494 & 0.015 & 0.030 & BCU I & ISP & 14.664 & 14.893 & 0.69500 & 1.000 & 1.000 & $\ldots$ \\
\hline J0922.8-3959 & PKS 0920-39 & 140.69341 & -39.99307 & 0.017 & 0.165 & BCU II & LSP & 13.775 & 13.775 & $\ldots$ & 0.999 & 0.948 & $\cdots$ \\
\hline J0940.7-6102 & MRC 0939-608 & 145.19733 & -61.12455 & 0.078 & 0.158 & BCU II & LSP & 13.671 & 13.671 & $\cdots$ & 0.984 & 0.897 & $\cdots$ \\
\hline J0956.7-6441 & AT20G J095612-643928 & 149.05075 & -64.65781 & 0.067 & 0.087 & BCU II & LSP & 13.285 & 13.285 & $\ldots$ & 0.928 & $\ldots$ & $\ldots$ \\
\hline J1005.0-4959 & PMN J1006-5018 & 151.55837 & -50.30374 & 0.370 & 0.197 & BCU II & LSP & 12.140 & 12.140 & $\cdots$ & $\cdots$ & 1.000 & $\cdots$ \\
\hline $\mathrm{J} 1015.2-4512$ & PMN J1014-4508 & 153.70981 & -45.14477 & 0.097 & 0.101 & BCU II & LSP & 12.025 & 12.025 & $\cdots$ & 0.986 & 0.900 & $\cdots$ \\
\hline J1038.9-5311 & MRC 1036-529 & 159.66941 & -53.19535 & 0.040 & 0.057 & BCU II & LSP & 12.235 & 12.235 & $\cdots$ & 0.998 & 1.000 & $\cdots$ \\
\hline J1047.8-6216 & PMN J1047-6217 & 161.92897 & -62.28740 & 0.016 & 0.044 & BCU II & LSP & 12.200 & 12.200 & $\cdots$ & 0.999 & 1.000 & $\cdots$ \\
\hline J1051.5-6517 & PKS 1049-650 & 162.84800 & -65.30240 & 0.017 & 0.063 & BCU II & ISP & 14.030 & 14.030 & $\cdots$ & 0.998 & $\cdots$ & $\cdots$ \\
\hline J1103.9-5357 & PKS 1101-536 & 165.96759 & -53.95019 & 0.007 & 0.028 & BLL & LSP & 13.830 & 13.830 & $\cdots$ & 0.999 & 0.984 & $\cdots$ \\
\hline J1123.2-6415 & AT20G J112319-641735 & 170.83090 & -64.29339 & 0.034 & 0.078 & BCU III & $\cdots$ & $\cdots$ & $\cdots$ & $\cdots$ & 0.995 & 0.931 & $\cdots$ \\
\hline J1136.6-6826 & PKS 1133-681 & 174.00874 & -68.45162 & 0.062 & 0.105 & BCU III & $\cdots$ & $\cdots$ & $\cdots$ & $\cdots$ & 0.987 & 0.932 & $\cdots$ \\
\hline J1229.8-5305 & AT20G J122939-530332 & 187.41637 & -53.05894 & 0.046 & 0.116 & BCU III & $\cdots$ & $\cdots$ & $\cdots$ & $\cdots$ & 0.991 & $\cdots$ & $\cdots$ \\
\hline J1233.9-5736 & AT20G J123407-573552 & 188.52933 & -57.59803 & 0.019 & 0.036 & BCU II & ISP & 14.700 & 14.700 & $\cdots$ & 0.998 & $\cdots$ & $\cdots$ \\
\hline J1256.1-5919 & PMN J1256-5919 & 194.02043 & -59.32886 & 0.013 & 0.054 & BCU III & $\cdots$ & $\cdots$ & $\cdots$ & $\cdots$ & 0.998 & $\cdots$ & $\cdots$ \\
\hline J1304.3-5535 & PMN J1303-5540 & 195.95507 & -55.67545 & 0.119 & 0.132 & BCU II & LSP & 12.725 & 12.725 & $\ldots$ & 0.974 & 0.908 & $\cdots$ \\
\hline J1308.1-6707 & PKS 1304-668 & 197.07240 & -67.11812 & 0.012 & 0.053 & BCU II & ISP & 14.230 & 14.230 & $\cdots$ & 0.998 & 0.973 & $\cdots$ \\
\hline J1315.1-5329 & PMN J1315-5334 & 198.76742 & -53.57663 & 0.089 & 0.069 & BCU I & LSP & 13.775 & 13.775 & $\ldots$ & 0.959 & 0.912 & $\ldots$ \\
\hline J1326.6-5256 & PMN J1326-5256 & 201.70512 & -52.93990 & 0.025 & 0.043 & BLL & LSP & 12.559 & 12.559 & $\ldots$ & 0.999 & 1.000 & $\ldots$ \\
\hline J1328.9-5607 & PMN J1329-5608 & 202.25477 & -56.13407 & 0.009 & 0.022 & $\mathrm{BCU} \mathrm{I}$ & LSP & 12.930 & 12.930 & $\cdots$ & 1.000 & 0.990 & $\cdots$ \\
\hline J1330.1-7002 & PKS 1326-697 & 202.54615 & -70.05363 & 0.008 & 0.031 & BCU II & LSP & 13.425 & 13.425 & $\ldots$ & 1.000 & 0.977 & $\ldots$ \\
\hline J1346.6-6027 & Cen B & 206.70435 & -60.40815 & 0.052 & 0.051 & RDG & ISP & 14.762 & 14.762 & 0.01292 & 1.000 & 1.000 & . \\
\hline J1353.5-6640 & 1RXS J135341.1-664002 & 208.41726 & -66.66602 & 0.011 & 0.037 & BLL & HSP & 15.700 & 15.700 & $\ldots$ & 1.000 & $\ldots$ & 0.963 \\
\hline J1400.7-5605 & PMN J1400-5605 & 210.17407 & -56.08210 & 0.009 & 0.121 & BCU II & LSP & 12.280 & 12.280 & $\ldots$ & 0.997 & $\ldots$ & $\ldots$ \\
\hline J1413.2-6518 & Circinus galaxy & 213.29172 & -65.34571 & 0.043 & 0.119 & sy & HSP & 15.440 & 15.440 & $\cdots$ & 0.988 & $\cdots$ & 0.886 \\
\hline J1419.1-5156 & PMN J1419-5155 & 214.89685 & -51.91627 & 0.079 & 0.143 & BCU II & LSP & 12.550 & 12.550 & $\ldots$ & 0.993 & 0.921 & $\ldots$ \\
\hline J1424.6-6807 & PKS 1420-679 & 216.23149 & -68.13280 & 0.027 & 0.059 & BCU II & LSP & 12.480 & 12.480 & $\cdots$ & 1.000 & 1.000 & $\ldots$ \\
\hline J1503.7-6426 & AT20G J150350-642539 & 225.95892 & -64.42764 & 0.025 & 0.046 & BCU II & LSP & 13.285 & 13.285 & $\cdots$ & 0.997 & $\cdots$ & $\cdots$ \\
\hline J1508.7-4956 & PMN J1508-4953 & 227.16227 & -49.88398 & 0.051 & 0.087 & BCU II & LSP & 11.780 & 11.780 & $\ldots$ & 0.999 & 0.956 & $\ldots$ \\
\hline J1514.5-4750 & PMN J1514-4748 & 228.66677 & -47.80829 & 0.032 & 0.063 & FSRQ & LSP & 12.515 & 12.922 & 1.55120 & 0.999 & 0.963 & $\cdots$ \\
\hline J1525.2-5905 & PMN J1524-5903 & 231.21301 & -59.06103 & 0.060 & 0.206 & BCU II & LSP & 12.655 & 12.655 & $\ldots$ & 0.986 & 0.848 & $\ldots$ \\
\hline J1558.9-6432 & PMN J1558-6432 & 239.70952 & -64.54157 & 0.012 & 0.030 & BLL & HSP & 15.300 & 15.333 & 0.07958 & 1.000 & 0.977 & 0.937 \\
\hline J1600.3-5810 & MRC 1556-580 & 240.05157 & -58.18416 & 0.020 & 0.078 & BCU III & $\cdots$ & $\cdots$ & $\cdots$ & & 0.998 & 0.952 & \\
\hline J1603.9-4903 & PMN J1603-4904 & 240.96119 & -49.06820 & 0.012 & 0.014 & BLL & ISP & 14.615 & 14.615 & $\ldots$ & 1.000 & 0.988 & $\ldots$ \\
\hline J1604.4-4442 & PMN J1604-4441 & 241.12925 & -44.69221 & 0.027 & 0.038 & BCU I & LSP & 12.947 & 12.947 & $\ldots$ & 0.999 & 1.000 & $\ldots$ \\
\hline J1610.6-3956 & PMN J1610-3958 & 242.59116 & -39.98287 & 0.061 & 0.183 & FSRQ & LSP & 13.088 & 13.269 & 0.51800 & 0.999 & 0.868 & $\cdots$ \\
\hline J1617.4-5846 & MRC 1613-586 & 244.32455 & -58.80218 & 0.041 & 0.073 & FSRQ & LSP & 12.550 & 12.550 & 1.42200 & 0.996 & 1.000 & 0.844 \\
\hline J1637.6-3449 & NVSS J163750-344915 & 249.46249 & -34.82098 & 0.039 & 0.042 & BCU II & LSP & 13.000 & 13.000 & $\cdots$ & 0.983 & 0.843 & 0.879 \\
\hline J1645.2-5747 & AT20G J164513-575122 & 251.30595 & -57.85622 & 0.067 & 0.109 & BCU III & $\cdots$ & $\cdots$ & $\cdots$ & $\cdots$ & 0.979 & $\cdots$ & $\cdots$ \\
\hline J1648.5-4829 & PMN J1648-4826 & 252.19968 & -48.43856 & 0.064 & 0.139 & BCU III & $\ldots$ & $\ldots$ & $\ldots$ & $\cdots$ & 0.993 & $\cdots$ & $\ldots$ \\
\hline J1650.2-5044 & PMN J1650-5044 & 252.56928 & -50.74673 & 0.004 & 0.023 & $\mathrm{BCU} \mathrm{I}$ & LSP & 12.725 & 12.725 & & 1.000 & 1.000 & $\cdots$ \\
\hline J1656.2-3303 & Swift J1656.3-3302 & 254.07025 & -33.03633 & 0.016 & 0.118 & FSRQ & LSP & 12.648 & 13.179 & 2.40000 & 1.000 & 0.883 & $\cdots$ \\
\hline $\mathrm{J} 1659.7-3132$ & NVSS J165949-313047 & 254.95383 & -31.51325 & 0.036 & 0.090 & BCU II & LSP & 13.110 & 13.110 & $\cdots$ & 0.998 & 0.858 & $\cdots$ \\
\hline J1711.5-5029 & PMN J1711-5028 & 257.92080 & -50.47150 & 0.023 & 0.079 & BCU II & LSP & 13.390 & 13.390 & $\cdots$ & 0.997 & $\cdots$ & $\cdots$ \\
\hline J1717.4-5157 & PMN J1717-5155 & 259.39455 & -51.92553 & 0.044 & 0.076 & FSRQ & LSP & 12.836 & 13.170 & 1.15800 & 0.990 & $\cdots$ & $\cdots$ \\
\hline J1717.8-3342 & TXS 1714-336 & 259.40012 & -33.70245 & 0.042 & 0.036 & BLL & LSP & 12.865 & 12.865 & $\cdots$ & 1.000 & 0.922 & $\cdots$ \\
\hline J1718.1-3056 & PMN J1718-3056 & 259.52173 & -30.93753 & 0.007 & 0.062 & BCU III & $\ldots$ & $\cdots$ & $\ldots$ & $\ldots$ & 0.998 & 0.880 & $\ldots$ \\
\hline J1731.8-3001 & NVSS J173146-300309 & 262.94538 & -30.05255 & 0.035 & 0.035 & BLL & $\ldots$ & $\ldots$ & $\ldots$ & $\ldots$ & 0.994 & 0.841 & $\ldots$ \\
\hline
\end{tabular}


Table 6

(Continued)

\begin{tabular}{|c|c|c|c|c|c|c|c|c|c|c|c|c|c|}
\hline $\begin{array}{l}\text { 3FGL Source } \\
\text { Name } \\
(1)\end{array}$ & $\begin{array}{l}\text { Counterpart } \\
\text { Name } \\
\text { (2) }\end{array}$ & $\begin{array}{l}\text { R.A. } \\
\left({ }^{\circ}\right) \\
(3)\end{array}$ & $\begin{array}{c}\text { Decl. } \\
\left({ }^{\circ}\right) \\
(4)\end{array}$ & $\begin{array}{c}\text { AngSep } \\
\left({ }^{\circ}\right) \\
(5)\end{array}$ & $\begin{array}{l}\theta_{95} \\
\left({ }^{\circ}\right) \\
(6)\end{array}$ & $\begin{array}{l}\text { Optical } \\
\text { Class } \\
(7)\end{array}$ & $\begin{array}{l}\text { SED } \\
\text { Class } \\
(8)\end{array}$ & $\begin{array}{c}\log \left(\nu_{\text {peak }}^{\text {S,meas }}\right) \\
(9)\end{array}$ & $\begin{array}{c}\log \left(\nu_{\text {peak }}^{\mathrm{S}}\right) \\
(10)\end{array}$ & $\begin{array}{c}z \\
(11)\end{array}$ & $\begin{array}{l}\text { Prob. } \\
\text { Bay. } \\
(12)\end{array}$ & $\begin{array}{c}\text { Rel. } \\
\mathrm{LR}_{\mathrm{RG}} \\
(13)\end{array}$ & $\begin{array}{c}\text { Rel. } \\
\text { LR }_{\mathrm{XC}} \\
(14)\end{array}$ \\
\hline J1741.9-2539 & NVSS J174154-253743 & 265.47687 & -25.62872 & 0.034 & 0.044 & BCU III & $\ldots$ & $\ldots$ & $\ldots$ & $\ldots$ & 0.994 & 0.813 & $\ldots$ \\
\hline J1744.9-1725 & 1RXS J174459.5-172640 & 266.24914 & -17.44348 & 0.011 & 0.037 & BCU III & $\ldots$ & $\ldots$ & $\ldots$ & $\ldots$ & $\ldots$ & 0.837 & 0.963 \\
\hline J1802.6-3940 & PMN J1802-3940 & 270.67783 & -39.66886 & 0.006 & 0.017 & FSRQ & LSP & 12.445 & 12.810 & 1.31900 & 1.000 & 0.985 & \\
\hline J1823.6-3453 & NVSS J182338-345412 & 275.91079 & -34.90334 & 0.009 & 0.024 & BCU II & HSP & 16.140 & 16.140 & $\ldots$ & 1.000 & 0.925 & 0.983 \\
\hline J1828.9-2417 & 1RXS J182853.8-241746 & 277.22879 & -24.29344 & 0.015 & 0.053 & BCU I & HSP & 16.456 & 16.456 & $\ldots$ & $\ldots$ & 0.872 & 0.914 \\
\hline $\mathrm{J} 1830.1+0617$ & TXS $1827+062$ & 277.52475 & 6.32110 & 0.032 & 0.045 & FSRQ & LSP & 12.305 & 12.547 & 0.74500 & 0.999 & 0.920 & $\ldots$ \\
\hline J1831.0-2714 & PMN J1831-2714 & 277.75019 & -27.23505 & 0.012 & 0.114 & BCU III & $\ldots$ & $\ldots$ & $\ldots$ & $\ldots$ & 0.994 & 0.810 & $\ldots$ \\
\hline J1833.6-2103 & PKS 1830-211 & 278.41619 & -21.06126 & 0.007 & 0.014 & FSRQ & LSP & 12.585 & 13.130 & 2.50700 & 1.000 & 0.994 & 0.939 \\
\hline J1835.4+1349 & TXS $1833+137$ & 278.89730 & 13.81354 & 0.039 & 0.118 & BCU III & $\ldots$ & $\ldots$ & $\ldots$ & $\ldots$ & 0.991 & $\ldots$ & $\ldots$ \\
\hline $\mathrm{J} 1844.3+1547$ & NVSS J184425+154646 & 281.10567 & 15.77940 & 0.022 & 0.039 & BCU II & ISP & 14.708 & 14.708 & $\ldots$ & 0.998 & 0.867 & $\ldots$ \\
\hline J1849.3-1645 & 1RXS J184919.7-164726 & 282.33110 & -16.78999 & 0.026 & 0.053 & BCU III & $\ldots$ & $\ldots$ & $\ldots$ & $\ldots$ & $\ldots$ & $\ldots$ & 0.907 \\
\hline J1908.8-0130 & NVSS J190836-012642 & 287.15393 & -1.44532 & 0.086 & 0.074 & BCU II & LSP & 11.782 & 11.782 & $\ldots$ & 0.996 & $\ldots$ & $\ldots$ \\
\hline $\mathrm{J} 1910.8+2855$ & 1RXS J191053.2+285622 & 287.71764 & 28.93926 & 0.012 & 0.048 & BCU II & HSP & 16.910 & 16.910 & $\ldots$ & $\ldots$ & $\ldots$ & 0.942 \\
\hline J1912.0-0804 & PMN J1912-0804 & 288.02970 & -8.07275 & 0.010 & 0.077 & BCU II & HSP & 15.050 & 15.050 & $\ldots$ & 0.999 & 0.905 & $\ldots$ \\
\hline $\mathrm{J} 1924.9+2817$ & NVSS J192502+281542 & 291.25942 & 28.26172 & 0.043 & 0.056 & BCU II & HSP & 15.850 & 15.850 & $\ldots$ & $\ldots$ & 0.800 & 0.906 \\
\hline $\mathrm{J} 1925.7+1228$ & TXS 1923+123 & 291.42007 & 12.46058 & 0.011 & 0.115 & BCU III & $\ldots$ & $\ldots$ & $\ldots$ & $\ldots$ & 0.992 & 0.808 & \\
\hline J1931.1+0937 & RX J1931.1+0937 & 292.78819 & 9.62119 & 0.010 & 0.020 & BLL & HSP & 16.150 & 16.150 & $\ldots$ & 1.000 & 0.860 & 0.980 \\
\hline J1933.4+0727 & 1RXS J193320.3+072616 & 293.33459 & 7.43941 & 0.030 & 0.074 & BCU II & HSP & 15.980 & 15.980 & $\ldots$ & 0.998 & 0.823 & 0.899 \\
\hline $\mathrm{J} 1942.7+1033$ & 1RXS J194246.3+103339 & 295.69785 & 10.55753 & 0.009 & 0.023 & BCU II & HSP & 15.435 & 15.435 & $\ldots$ & 1.000 & 0.917 & 0.958 \\
\hline $\mathrm{J} 1949.0+1312$ & 87 GB $194635.4+130713$ & 297.23037 & 13.24400 & 0.043 & 0.051 & BCU II & HSP & 15.450 & 15.450 & $\ldots$ & $\ldots$ & 0.836 & $\ldots$ \\
\hline $\mathrm{J} 1955.1+1357$ & 87 GB $195252.4+135009$ & 298.79821 & 13.97118 & 0.017 & 0.052 & FSRQ & LSP & 12.865 & 13.106 & 0.74300 & 1.000 & 0.885 & $\ldots$ \\
\hline $\mathrm{J} 2000.1+4212$ & MG4 J195957+4213 & 299.99487 & 42.22965 & 0.032 & 0.063 & BCU II & LSP & 12.550 & 12.550 & $\ldots$ & 0.997 & 0.897 & $\ldots$ \\
\hline $\mathrm{J} 2001.1+4352$ & MG4 J200112+4352 & 300.30364 & 43.88134 & 0.005 & 0.012 & BLL & HSP & 15.205 & 15.205 & $\ldots$ & 1.000 & 0.944 & $\ldots$ \\
\hline $\mathrm{J} 2012.0+4629$ & 7C 2010+4619 & 303.02349 & 46.48216 & 0.020 & 0.026 & BLL & ISP & 14.958 & 14.958 & $\ldots$ & 1.000 & 0.954 & 0.967 \\
\hline J2015.6+3709 & MG2 J201534+3710 & 303.86971 & 37.18320 & 0.038 & 0.027 & FSRQ & LSP & 12.743 & 13.012 & 0.85900 & 0.994 & 0.961 & $\ldots$ \\
\hline $\mathrm{J} 2018.5+3851$ & TXS 2016+386 & 304.62927 & 38.85538 & 0.005 & 0.048 & BCU II & LSP & 13.508 & 13.508 & $\ldots$ & 0.998 & 0.910 & $\ldots$ \\
\hline $\mathrm{J} 2023.2+3154$ & $4 C+31.56$ & 305.82924 & 31.88397 & 0.028 & 0.090 & BCU I & LSP & 13.382 & 13.514 & 0.35600 & 0.998 & 0.967 & $\ldots$ \\
\hline $\mathrm{J} 2025.2+3340$ & B2 $2023+33$ & 306.29518 & 33.71673 & 0.053 & 0.052 & BCU I & LSP & 12.305 & 12.391 & 0.21900 & 0.999 & 0.942 & $\ldots$ \\
\hline J2029.4+4923 & MG4 J202932+4925 & 307.41614 & 49.43949 & 0.055 & 0.058 & BLL & LSP & 13.320 & 13.320 & & 0.968 & $\ldots$ & $\ldots$ \\
\hline $\mathrm{J} 2038.8+5113$ & 3C 418 & 309.65431 & 51.32018 & 0.104 & 0.110 & FSRQ & LSP & 12.480 & 12.909 & 1.68600 & 0.996 & 0.962 & $\ldots$ \\
\hline $\mathrm{J} 2039.5+5217$ & 1ES $2037+521$ & 309.84799 & 52.33056 & 0.043 & 0.062 & BLL & HSP & 16.448 & 16.470 & 0.05300 & 1.000 & $\ldots$ & 0.895 \\
\hline $\mathrm{J} 2056.7+4938$ & RGB J2056+496 & 314.17808 & 49.66850 & 0.027 & 0.027 & BCU II & HSP & 15.742 & 15.742 & $\ldots$ & 0.995 & 0.894 & 0.957 \\
\hline $\mathrm{J} 2108.0+3654$ & TXS 2106+367 & 317.02275 & 36.92404 & 0.018 & 0.059 & BCU II & ISP & 14.860 & 14.860 & $\ldots$ & $\ldots$ & 0.837 & $\ldots$ \\
\hline $\mathrm{J} 2110.3+3540$ & B2 $2107+35 \mathrm{~A}$ & 317.38283 & 35.54933 & 0.203 & 0.246 & BCU II & ISP & 14.048 & 14.048 & $\ldots$ & 0.989 & 0.862 & 0.836 \\
\hline J2201.7+5047 & NRAO 676 & 330.43141 & 50.81566 & 0.016 & 0.042 & FSRQ & LSP & 12.515 & 12.977 & 1.89900 & 1.000 & 0.955 & $\ldots$ \\
\hline $\mathrm{J} 2347.0+5142$ & 1ES $2344+514$ & 356.77015 & 51.70497 & 0.005 & 0.018 & BLL & HSP & 15.850 & 15.869 & 0.04400 & 1.000 & 0.953 & 0.980 \\
\hline $\mathrm{J} 2347.9+5436$ & NVSS J234753+543627 & 356.97138 & 54.60754 & 0.007 & 0.066 & BCU II & HSP & 16.400 & 16.400 & & $\ldots$ & $\ldots$ & 0.925 \\
\hline
\end{tabular}

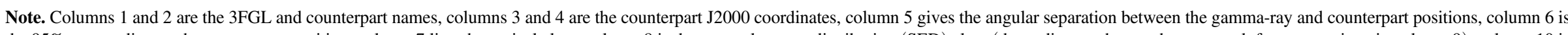

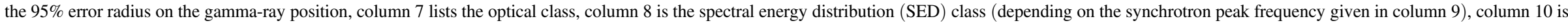

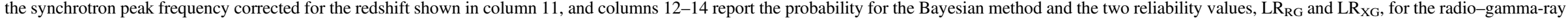
match and the X-ray-gamma-ray match, respectively.

(This table is available in machine-readable form.) 
Table 7

Sources from Earlier FGL Catalogs Missing in 3LAC

\begin{tabular}{|c|c|c|c|c|c|c|c|}
\hline $\begin{array}{l}\text { Counterpart } \\
\text { Name } \\
(1)\end{array}$ & $\begin{array}{c}\text { R.A. } \\
\left({ }^{\circ}\right) \\
(2)\end{array}$ & $\begin{array}{c}\text { Decl. } \\
\left({ }^{\circ}\right) \\
(3)\end{array}$ & $\begin{array}{l}\text { Optical } \\
\text { Class } \\
(4)\end{array}$ & $\begin{array}{c}\text { SED } \\
\text { Class } \\
(5)\end{array}$ & $\begin{array}{l}\text { Redshift } \\
\text { (6) }\end{array}$ & $\begin{array}{c}\text { 1FGL } \\
\text { Name } \\
(7)\end{array}$ & $\begin{array}{c}2 \mathrm{FGL} \\
\text { Name } \\
(8)\end{array}$ \\
\hline CRATES J0009+0628 & 2.26638 & 6.47256 & BLL & LSP & $\cdots$ & 1FGL J0008.9+0635 & $\cdots$ \\
\hline CGRaBS J0011+0057 & 2.87667 & 0.96439 & FSRQ & LSP & 1.492 & 1FGL J0011.1+0050 & $\ldots$ \\
\hline GB6 J0013+1910 & 3.48509906 & 19.1782456 & BLL & $\cdots$ & 0.473 & $\cdots$ & 2FGL J0013.8+1907 \\
\hline PKS 0056-572 & 14.6940417 & -56.98675 & $\mathrm{BCU}$ & LSP & $\cdots$ & & 2FGL J0059.7-5700 \\
\hline PKS 0116-219 & 19.73858 & -21.69167 & FSRQ & LSP & 1.165 & 1FGL J0118.7-2137 & $\cdots$ \\
\hline TXS 0154-244 & 29.1237238 & -24.2146961 & $\mathrm{BCU}$ & $\cdots$ & $\cdots$ & $\cdots$ & 2FGL J0156.5-2419 \\
\hline S5 $0159+723$ & 30.8883864 & 72.5483361 & BLL & LSP & $\cdots$ & 1FGL J0203.5+7234 & 2FGL J0203.6+7235 \\
\hline B2 $0200+30$ & 30.93898 & 30.69142 & $\ldots$ & $\ldots$ & $\ldots$ & 1FGL J0203.5+3044 & $\ldots$ \\
\hline 1RXS J021905.8-172503 & 34.772989 & -17.4204904 & BLL & HSP & 0.128 & $\cdots$ & 2FGL J0219.1-1725 \\
\hline NGC 1068 & 40.6696759 & -0.013268027 & starburst & $\ldots$ & 0.00419 & $\ldots$ & 2FGL J0242.5+0006 \\
\hline CRATES J0258+2030 & 44.53046 & 20.50044 & BLL & LSP & $\cdots$ & 1FGL J0258.0+2033 & $\cdots$ \\
\hline CRATES J0305-0607 & 46.25238 & -6.12819 & BLL & $\cdots$ & $\cdots$ & 1FGL J0305.0-0601 & $\cdots$ \\
\hline NVSS J033223-111951 & 53.0970024 & -11.3309802 & $\mathrm{BCU}$ & HSP & $\cdots$ & $\cdots$ & 2FGL J0332.5-1118 \\
\hline PMN J0413-5332 & 63.30629 & -53.53361 & FSRQ & $\cdots$ & 1.027 & 1FGL J0413.4-5334 & 2FGL J0413.5-5332 \\
\hline PKS 0420+022 & 65.71754 & 2.32414 & FSRQ & LSP & 2.277 & 1FGL J0422.1+0211 & $\cdots$ \\
\hline GB6 J0437+6757 & 69.3862329 & 67.9544125 & $\mathrm{BCU}$ & $\cdots$ & $\cdots$ & $\cdots$ & 2FGL J0436.2+6759 \\
\hline PKS 0437-322 & 69.8915847 & -32.169605 & $\mathrm{BCU}$ & LSP & $\cdots$ & $\cdots$ & 2FGL J0440.1-3211 \\
\hline TXS $0437+145$ & 70.0879763 & 14.6324959 & $\mathrm{BCU}$ & $\cdots$ & $\cdots$ & $\cdots$ & 2FGL J0440.4+1433 \\
\hline PKS 0440-00 & 70.6609229 & -0.295256342 & FSRQ & LSP & 0.844 & $\ldots$ & 2FGL J0442.7-0017 \\
\hline $4 \mathrm{C}+06.21$ & 74.28212 & 6.75203 & FSRQ & LSP & 0.405 & 1FGL J0457.9+0649 & $\ldots$ \\
\hline 1WGA J0506.6-0857 & 76.6662037 & -8.96713326 & BLL & HSP & $\cdots$ & $\cdots$ & 2FGL J0506.5-0901 \\
\hline PMN J0507-6104 & 76.9772083 & -61.0786389 & FSRQ & $\cdots$ & 1.089 & $\cdots$ & 2FGL J0507.5-6102 \\
\hline PKS 0514-459 & 78.9367917 & -45.9455 & FSRQ & LSP & 0.194 & $\cdots$ & 2FGL J0516.5-4601 \\
\hline OG 050 & 83.1625 & 7.54536 & FSRQ & LSP & 1.254 & 1FGL J0532.9+0733 & $\cdots$ \\
\hline PMN J0533-7216 & 83.4342917 & -72.2730278 & $\mathrm{BCU}$ & $\cdots$ & $\cdots$ & $\cdots$ & 2FGL J0532.5-7223 \\
\hline FRBA J0536-3343 & 84.12131 & -33.71737 & BLL & HSP & $\cdots$ & 1FGL J0536.2-3348 & $\cdots$ \\
\hline SUMSS J053748-571828 & 84.4532083 & -57.3080278 & $\mathrm{BCU}$ & ISP & $\cdots$ & $\cdots$ & 2FGL J0537.7-5716 \\
\hline CRATES J0539-0356 & 84.81454 & -3.94892 & $\cdots$ & $\cdots$ & $\cdots$ & 1FGL J0539.4-0400 & $\cdots$ \\
\hline PKS 0539-057 & 85.40867 & -5.69706 & FSRQ & LSP & 0.839 & 1FGL J0540.9-0547 & $\cdots$ \\
\hline PMN J0608-1520 & 92.0062923 & -15.3436112 & FSRQ & LSP & 1.094 & 1FGL J0608.0-1521 & 2FGL J0608.0-1521 \\
\hline PMN J0610-1847 & 92.5746761 & -18.7944297 & BLL & LSP & $\cdots$ & $\ldots$ & 2FGL J0609.6-1847 \\
\hline CGRaBS J0634-2335 & 98.7459773 & -23.5866986 & FSRQ & $\ldots$ & 1.535 & $\ldots$ & 2FGL J0635.0-2334 \\
\hline BZU J0645+6024 & 101.25571 & 60.41175 & AGN & $\cdots$ & 0.832 & 1FGL J0645.5+6033 & $\ldots$ \\
\hline PKS 0700-465 & 105.39392 & -46.57683 & FSRQ & LSP & 0.822 & 1FGL J0702.0-4628 & $\cdots$ \\
\hline MG2 J071354+1934 & 108.482006 & 19.5837737 & FSRQ & LSP & 0.54 & 1FGL J0714.0+1935 & 2FGL J0714.0+1933 \\
\hline BZB J0723+5841 & 110.80817 & 58.68844 & BLL & HSP & $\cdots$ & 1FGL J0722.3+5837 & $\ldots$ \\
\hline $4 \mathrm{C}+14.23$ & 111.32004 & 14.42047 & FSRQ & $\ldots$ & 1.038 & 1FGL J0725.3+1431 & $\ldots$ \\
\hline 1RXS J073026.0+330727 & 112.608899 & 33.1227122 & BLL & HSP & 0.112 & 1FGL J0730.0+3305 & 2FGL J0729.9+3304 \\
\hline CGRaBS J0814+6431 & 123.66329 & 64.52278 & BLL & ISP & $\cdots$ & 1FGL J0815.0+6434 & $\cdots$ \\
\hline RX J0817.9+3243 & 124.461462 & 32.7277131 & BLL & HSP & $\cdots$ & $\ldots$ & 2FGL J0817.9+3238 \\
\hline RX J0819.2-0756 & 124.822975 & -7.9411836 & BLL & HSP & $\cdots$ & $\cdots$ & 2FGL J0819.6-0803 \\
\hline $4 C+39.23$ & 126.230974 & 39.2782392 & FSRQ & LSP & 1.216 & $\ldots$ & 2FGL J0824.7+3914 \\
\hline BZB J0842+0252 & 130.6063 & 2.88131 & BLL & HSP & 0.425 & 1FGL J0842.2+0251 & $\ldots$ \\
\hline TXS 0845-068 & 131.986571 & -7.05500701 & BLL & ISP & $\cdots$ & $\cdots$ & 2FGL J0848.1-0703 \\
\hline GB6 J0850+4855 & 132.501843 & 48.9162398 & BLL & ISP & $\cdots$ & 1FGL J0849.9+4852 & 2FGL J0849.8+4852 \\
\hline GB6 J0856+7146 & 134.228226 & 71.7735317 & $\mathrm{BCU}$ & LSP & $\cdots$ & $\cdots$ & 2FGL J0856.0+7136 \\
\hline B3 $0908+416 B$ & 138.048527 & 41.4358885 & FSRQ & LSP & 2.563 & 1FGL J0912.3+4127 & 2FGL J0912.1+4126 \\
\hline OK 630 & 140.40096 & 62.2645 & FSRQ & LSP & 1.446 & 1FGL J0919.6+6216 & $\ldots$ \\
\hline GB6 J0922+0433 & 140.612897 & 4.56042681 & $\mathrm{BCU}$ & $\ldots$ & $\cdots$ & $\ldots$ & 2FGL J0922.7+0435 \\
\hline GB6 J0934+3926 & 143.527628 & 39.4424247 & BLL & $\cdots$ & $\cdots$ & 1FGL J0934.5+3929 & 2FGL J0934.7+3932 \\
\hline RX J0940.3+6148 & 145.093673 & 61.8069546 & BLL & HSP & 0.211 & 1FGL J0941.2+6149 & 2FGL J0941.4+6148 \\
\hline BZB J0952+3936 & 148.06129 & 39.60442 & BLL & HSP & $\cdots$ & 1FGL J0952.2+3926 & $\ldots$ \\
\hline OK 290 & 149.207825 & 25.2543968 & FSRQ & LSP & 0.707969 & 1FGL J0956.9+2513 & 2FGL J0956.9+2516 \\
\hline PKS 1004-217 & 151.69338 & -21.989 & FSRQ & LSP & 0.33 & 1FGL J1007.1-2157 & $\ldots$ \\
\hline PKS 1008-01 & 152.715672 & -2.00533479 & FSRQ & $\ldots$ & 0.887 & 1FGL J1011.0-0156 & 2FGL J1010.8-0158 \\
\hline $4 \mathrm{C}+23.24$ & 153.696062 & 23.0201609 & FSRQ & LSP & 0.566 & $\ldots$ & 2FGL J1014.1+2306 \\
\hline PKS 1021-323 & 156.001761 & -32.570915 & FSRQ & $\cdots$ & 1.568 & $\ldots$ & 2FGL J1023.8-3248 \\
\hline S5 $1039+81$ & 161.096173 & 80.911074 & FSRQ & LSP & 1.26 & 1FGL J1048.7+8054 & 2FGL J1042.6+8053 \\
\hline GB6 J1049+1548 & 162.413685 & 15.8105625 & $\mathrm{BCU}$ & $\ldots$ & $\cdots$ & $\ldots$ & 2FGL J1049.4+1551 \\
\hline 1RXS J112551.6-074219 & 171.466497 & -7.70598752 & BLL & HSP & 0.279 & 1FGL J1126.0-0741 & 2FGL J1126.0-0743 \\
\hline PKS 1124-186 & 171.768399 & -18.9550553 & FSRQ & LSP & 1.048 & $\ldots$ & 2FGL J1126.6-1856 \\
\hline PKS 1133-739 & 174.039417 & -74.2635 & $\mathrm{BCU}$ & $\cdots$ & $\cdots$ & $\cdots$ & 2FGL J1134.4-7415 \\
\hline
\end{tabular}


Table 7

(Continued)

\begin{tabular}{|c|c|c|c|c|c|c|c|}
\hline $\begin{array}{l}\text { Counterpart } \\
\text { Name } \\
(1)\end{array}$ & $\begin{array}{c}\text { R.A. } \\
\left({ }^{\circ}\right) \\
(2)\end{array}$ & $\begin{array}{c}\text { Decl. } \\
\left({ }^{\circ}\right) \\
(3)\end{array}$ & $\begin{array}{l}\text { Optical } \\
\text { Class } \\
(4)\end{array}$ & $\begin{array}{c}\text { SED } \\
\text { Class } \\
(5)\end{array}$ & Redshift & $\begin{array}{c}\text { 1FGL } \\
\text { Name } \\
(7)\end{array}$ & $\begin{array}{c}2 \mathrm{FGL} \\
\text { Name } \\
(8)\end{array}$ \\
\hline S4 1144+40 & 176.742963 & 39.9763205 & FSRQ & $\ldots$ & 1.0882 & 1FGL J1146.8+4004 & 2FGL J1146.9+4000 \\
\hline PKS 1217+02 & 185.051316 & 2.06154225 & FSRQ & ISP & 0.241 & $\ldots$ & 2FGL J1219.7+0201 \\
\hline PMN J1226-1328 & 186.726778 & -13.4774552 & BLL & $\ldots$ & 0.456 & 1FGL J1226.7-1332 & 2FGL J1226.7-1331 \\
\hline B2 $1229+29$ & 187.93158 & 28.79717 & BLL & ISP & 0.236 & 1FGL J1231.6+2850 & $\ldots$ \\
\hline 5C 12.170 & 195.371521 & 33.6168978 & $\mathrm{BCU}$ & $\cdots$ & 1.00913 & $\cdots$ & 2FGL J1301.6+3331 \\
\hline NGC 4945 & 196.36446 & -49.46806 & AGN & $\ldots$ & 0.002 & 1FGL J1305.4-4928 & $\ldots$ \\
\hline $\mathrm{OP}-034$ & 200.65379 & -9.62717 & FSRQ & $\cdots$ & 1.864 & 1FGL J1322.7-0943 & $\ldots$ \\
\hline 1RXS 132928.0-053132 & 202.366669 & -5.52568984 & AGN & $\ldots$ & 0.575868 & $\ldots$ & 2FGL J1329.3-0528 \\
\hline 1ES $1421+582$ & 215.66206 & 58.03208 & BLL & HSP & $\ldots$ & 1FGL J1422.2+5757 & $\ldots$ \\
\hline CLASS J1423+3737 & 215.76921 & 37.62516 & BLL & $\ldots$ & $\ldots$ & 1FGL J1422.7+3743 & $\ldots$ \\
\hline PMN J1509-4340 & 227.398167 & -43.6753333 & FSRQ & LSP & 0.776 & $\ldots$ & 2FGL J1508.9-4342 \\
\hline CLASS J1537+8154 & 234.25036 & 81.90862 & $\ldots$ & $\ldots$ & $\cdots$ & 1FGL J1536.6+8200 & $\ldots$ \\
\hline 1ES $1544+820$ & 235.065419 & 81.918194 & BLL & HSP & $\cdots$ & $\ldots$ & 2FGL J1538.1+8159 \\
\hline $4 C-06.46$ & 246.13717 & -6.83047 & $\cdots$ & $\ldots$ & $\cdots$ & 1FGL J1624.7-0642 & $\ldots$ \\
\hline NGC 6251 & 248.13325 & 82.53789 & AGN & $\ldots$ & 0.025 & 1FGL J1635.4+8228 & $\ldots$ \\
\hline PMN J1657-1021 & 254.386346 & -10.3545458 & $\mathrm{BCU}$ & $\ldots$ & $\cdots$ & $\ldots$ & 2FGL J1657.1-1027 \\
\hline CGRaBS J1703-6212 & 255.901667 & -62.2111667 & FSRQ & $\cdots$ & 1.747 & $\ldots$ & 2FGL J1703.2-6217 \\
\hline PKS 1728+004 & 262.64583 & 0.41075 & FSRQ & $\ldots$ & 1.335 & 1FGL J1730.4+0008 & $\ldots$ \\
\hline CRATES J1803+0341 & 270.9845 & 3.68544 & FSRQ & $\cdots$ & 1.42 & 1FGL J1804.1+0336 & $\ldots$ \\
\hline 87 GB $181007.0+533142$ & 272.797216 & 53.5403097 & $\mathrm{BCU}$ & $\ldots$ & $\cdots$ & $\ldots$ & 2FGL J1811.0+5340 \\
\hline NVSS J181118+034114 & 272.825356 & 3.68726303 & BLL & HSP & $\ldots$ & $\cdots$ & 2FGL J1811.3+0339 \\
\hline PMN J1814-6412 & 273.65 & -64.2148056 & $\mathrm{BCU}$ & $\ldots$ & $\ldots$ & $\ldots$ & 2FGL J1815.6-6407 \\
\hline PMN J1816-4943 & 274.233125 & -49.7291389 & $\mathrm{BCU}$ & $\cdots$ & $\cdots$ & $\cdots$ & 2FGL J1816.7-4942 \\
\hline 87 GB $182712.0+272717$ & 277.308371 & 27.4841929 & $\mathrm{BCU}$ & $\ldots$ & $\ldots$ & $\ldots$ & 2FGL J1829.1+2725 \\
\hline B2 $1846+32 A$ & 282.09208 & 32.31739 & FSRQ & LSP & 0.798 & 1FGL J1848.5+3224 & $\ldots$ \\
\hline TXS 1918-126 & 290.349727 & -12.5317721 & BLL & $\ldots$ & $\cdots$ & 1FGL J1921.1-1234 & 2FGL J1921.3-1231 \\
\hline CRATES J1925-1018 & 291.26333 & -10.30344 & BLL & $\ldots$ & $\ldots$ & 1FGL J1925.1-1018 & $\ldots$ \\
\hline NGC 6814 & 295.668824 & -10.322184 & Seyfert & $\ldots$ & 0.0052 & $\ldots$ & 2FGL J1942.5-1024 \\
\hline 3C 407 & 302.10161 & -4.30814 & AGN & $\ldots$ & 0.589 & 1FGL J2008.6-0419 & $\ldots$ \\
\hline $4 C+72.28$ & 302.468826 & 72.4887054 & BLL & LSP & $\cdots$ & 1FGL J2009.1+7228 & 2FGL J2009.7+7225 \\
\hline PKS 2012-017 & 303.81317 & -1.62569 & BLL & $\ldots$ & $\cdots$ & 1FGL J2015.3-0129 & $\ldots$ \\
\hline CGRaBS J2022+7611 & 305.64829 & 76.19061 & BLL & $\ldots$ & $\ldots$ & 1FGL J2020.4+7608 & $\cdots$ \\
\hline CGRaBS J2025-2845 & 306.47337 & -28.76353 & $\ldots$ & LSP & $\ldots$ & 1FGL J2025.9-2852 & $\cdots$ \\
\hline SDSS J205528.20-002117.2 & 313.86749 & -0.35472 & BLL & HSP & $\cdots$ & 1FGL J2055.5-0023 & $\ldots$ \\
\hline PKS 2130-654 & 323.554542 & -65.227 & $\mathrm{BCU}$ & $\ldots$ & $\cdots$ & $\ldots$ & 2FGL J2134.5-6513 \\
\hline RBS 1769 & 324.719865 & -20.8962717 & BLL & HSP & 0.29 & $\ldots$ & 2FGL J2139.1-2054 \\
\hline $4 C+06.69$ & 327.022834 & 6.96092391 & FSRQ & LSP & 0.999 & 1FGL J2148.5+0654 & 2FGL J2148.2+0659 \\
\hline CRATES J2212+0646 & 333.21183 & 6.76908 & FSRQ & $\ldots$ & 1.121 & 1FGL J2212.9+0654 & $\ldots$ \\
\hline NVSS J222329+010226 & 335.8732293 & 1.04070536 & $\mathrm{BCU}$ & $\ldots$ & $\ldots$ & $\ldots$ & 2FGL J2223.4+0104 \\
\hline 1RXS 224642.0-520638 & 341.67575 & -52.1114167 & $\mathrm{BCU}$ & HSP & 0.194 & $\ldots$ & 2FGL J2246.8-5203 \\
\hline PKS 2244-002 & 341.875756 & 0.001971181 & BLL & ISP & 0.949 & 1FGL J2247.3+0000 & 2FGL J2247.2-0002 \\
\hline PKS 2320-021 & 350.76929 & -1.84669 & FSRQ & $\cdots$ & 1.774 & 1FGL J2322.3-0153 & $\ldots$ \\
\hline PKS 2325-408 & 352.080917 & -40.5858333 & $\mathrm{BCU}$ & $\ldots$ & $\ldots$ & $\ldots$ & 2FGL J2327.9-4037 \\
\hline PKS 2329-16 & 352.911061 & -15.949355 & FSRQ & LSP & 1.153 & $\ldots$ & 2FGL J2331.8-1607 \\
\hline CGRaBS J2345-1555 & 356.30192 & -15.91883 & FSRQ & LSP & 0.621 & 1FGL J2344.6-1554 & $\ldots$ \\
\hline
\end{tabular}

Note. Column 1 is the counterpart name, columns 2 and 3 are the counterpart J2000 coordinates, column 4 lists the optical class, column 5 is the spectral energy distribution (SED) class (depending on the synchrotron peak frequency), column 6 is the counterpart redshift, and columns 7 and 8 show the names in previous Fermi-LAT catalogs.

(This table is available in machine-readable form.)

and 9 other AGNs or BCUs detected in flaring, hardening, or enhanced activity states. Only one, PKS 1915-458 $(z=2.47$, ATel\#2666 and ATel\#2679) is not listed in the 3FGL/3LAC or in previous LAT catalogs. This high-redshift FSRQ appears to only emit gamma-rays sporadically within short time intervals.

In addition, three LAT sources announced in ATels and not present in the 3LAC might have extragalactic source associations: Fermi J0052+1110 located at high Galactic latitude),
PMN J1626-2426 (FSRQ in the vicinity of 3FGL J1626.2 -2428 but outside its error ellipse and located behind an $\mathrm{H}$ II region), and PMN J0623-3350 (flat spectrum radio source reported as Fermi J0623-3351). A fourth LAT ATel source tentatively associated there with the FSRQ PKS 2136-642 is listed as 3FGL J2141.6-6412 in 3FGL but is now associated with the BCU PMN J2141-6411 that is separated $\sim 15^{\prime}$ from the former. 


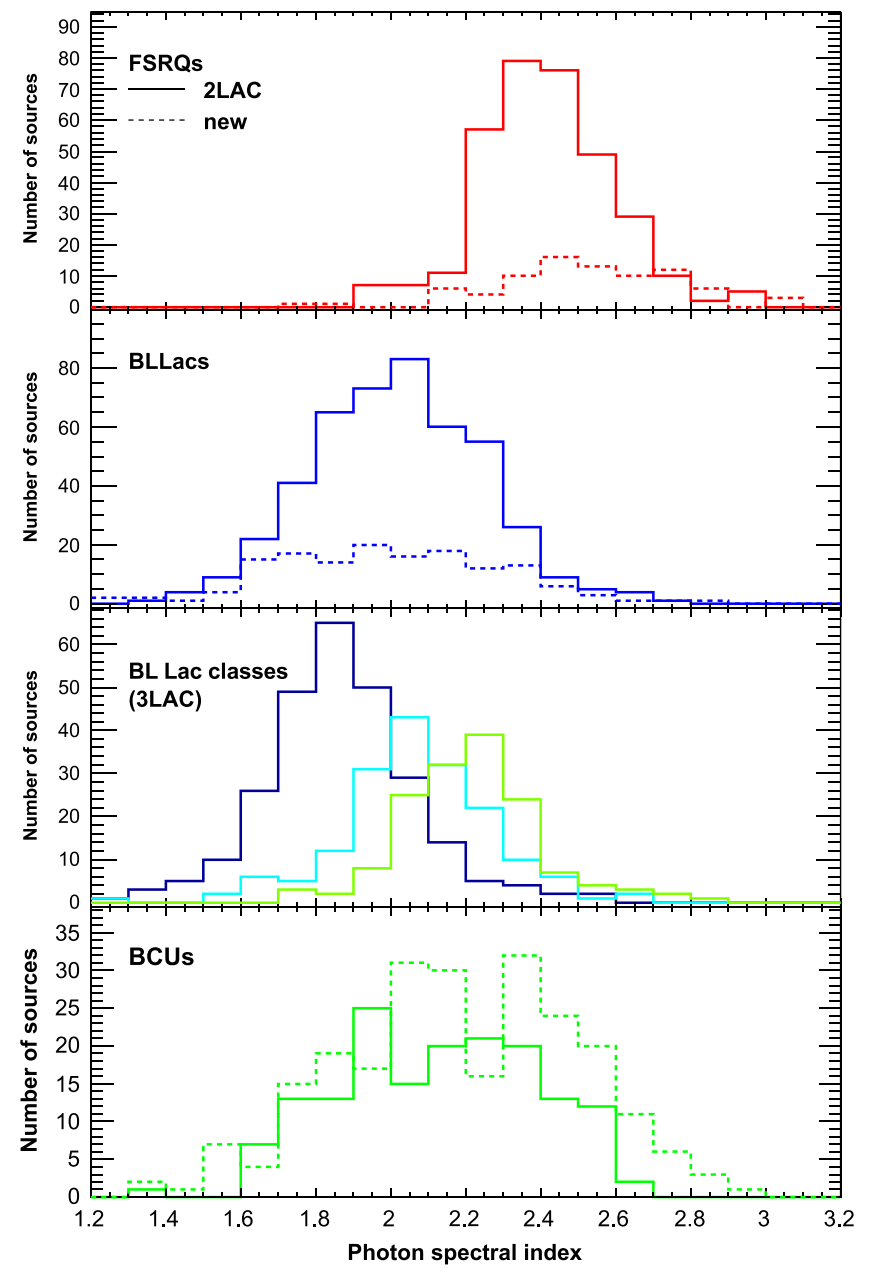

Figure 7. Photon spectral index distributions. Top: FSRQs (solid: 2LAC sources; dashed: new 3LAC sources). Second from top: BL Lacs (solid: 2LAC sources; dashed: new 3LAC sources). Third from top: 3LAC LSP-BL Lacs (green), ISP-BL Lacs (light blue), and HSP-BL Lacs (dark blue). Bottom: blazars of unknown type (solid: 2LAC sources; dashed: new 3LAC sources).

\section{PROPERTIES OF 3LAC SOURCES}

\subsection{Flux and Photon Spectral Index}

Figure 7 displays the photon index distributions for the different blazar classes both for the sources previously listed in 2LAC and the newly detected sources. The newly detected FSRQs are slightly softer than the 2 LAC ones $(2.53 \pm 0.03$ versus $2.41 \pm 0.01$ ), indicating that the LAT gradually detects more lower energy-peaked blazars. In contrast, there is no significant spectral difference between the two sets of BL Lacs. For BCUs, the distribution of the new sources extends further out on the high-index end $(\Gamma>2.4)$, where the overlap with the BL Lac distribution becomes very small. The corresponding sources seem likely to be FSRQs.

Figures 8 and 9 show the photon index versus the photon flux and energy flux, respectively, together with estimated flux limits. As noted in 2LAC, the strong bias observed toward hard sources in the photon-flux limit essentially vanishes when considering the energy-flux limit above $100 \mathrm{MeV}$ instead. (Note that this feature holds only for a lower bound of $100 \mathrm{MeV}$; other lower energy limits will bring about a dependence of the energy-flux limit on the spectral index.)

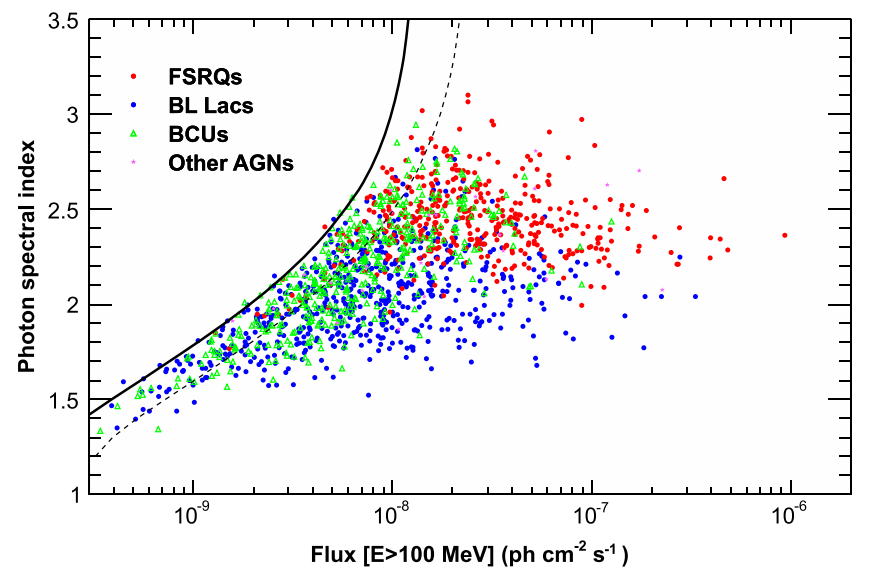

Figure 8. Photon spectral index vs. photon flux above $100 \mathrm{MeV}$ for blazars in the Clean Sample. Red circles: FSRQs; blue circles: BL Lacs; green triangles: blazars of unknown type; magenta stars: other AGNs. The solid (dashed) curve represents the approximate 3FGL (2FGL) detection limit based on a typical exposure.

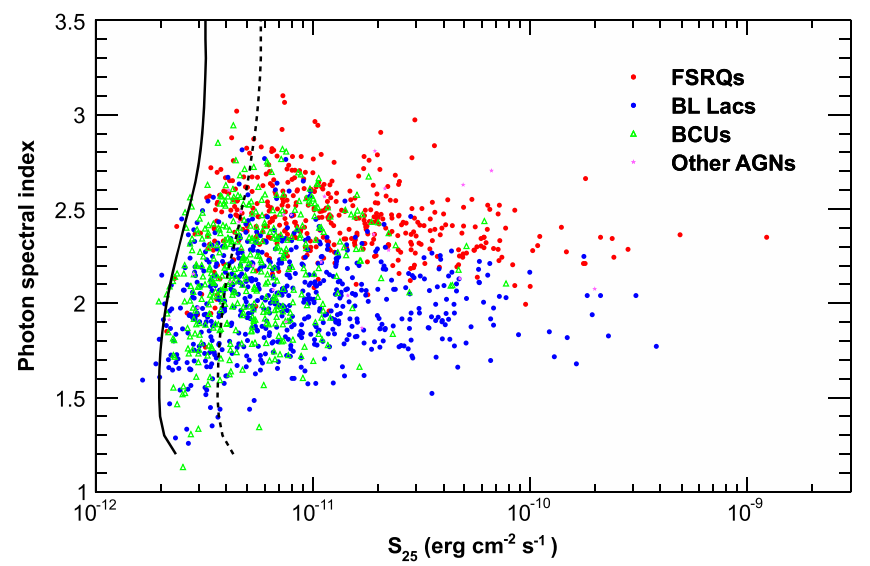

Figure 9. Photon spectral index vs. energy flux between $100 \mathrm{MeV}$ and $100 \mathrm{GeV}, \mathrm{S}_{25}$. Red circles: FSRQs; blue circles: BL Lacs; green triangles: blazars of unknown type; magenta stars: other AGNs. The solid (dashed) curve represents the approximate 3FGL (2FGL) detection limit based on a typical exposure.

Figure 10 shows the position of the synchrotron peak $\nu_{\text {peak,meas }}^{\mathrm{S}}$ versus the photon spectral index for FSRQs and BL Lacs with measured redshifts. The strong anticorrelation already observed in $1 \mathrm{LAC}$ and $2 \mathrm{LAC}$ is confirmed. Fitting a linear function $\Gamma=A+B \log \left(\nu_{\text {peak,rest }}^{\mathrm{S}} / 10^{14} \mathrm{~Hz}\right)$ yields $A=2.25 \pm 0.04$ and $B=-0.18 \pm 0.03$. The mean and rms of the $\Gamma$ distributions are $2.44 \pm 0.20,2.01 \pm 0.25,2.21 \pm$ $0.18,2.07 \pm 0.20$, and $1.87 \pm 0.20$ for FSRQs, the whole BL Lac sample, LSP-, ISP- and HSP-BL Lacs, respectively. FSRQs are overwhelmingly of the LSP class, so no distinction between SED-based classes will be made for them in figures and tallies. Only 37 FSRQs are of the ISP class and only 2 of the HSP class (BZB J0202+0849 and NVSS J025037+171209 associated with 3FGL J0202.3+0851 and 3FGL J0250.6+1713, respectively). As is visible in Figure 10, most ISP-FSRQs have softer spectra than the bulk of ISP-BL Lacs $(\langle\Gamma\rangle=2.40 \pm 0.04$ versus $2.07 \pm 0.02)$. In contrast, the two HSP-FSRQs have spectra $(\langle\Gamma\rangle=2.01)$ on par with the HSP-BL Lacs and thus much harder than the spectra of most other FSRQs. A similar trend is actually observed for BCUs, as can be seen in Figure 11, where the photon spectral index is plotted versus 


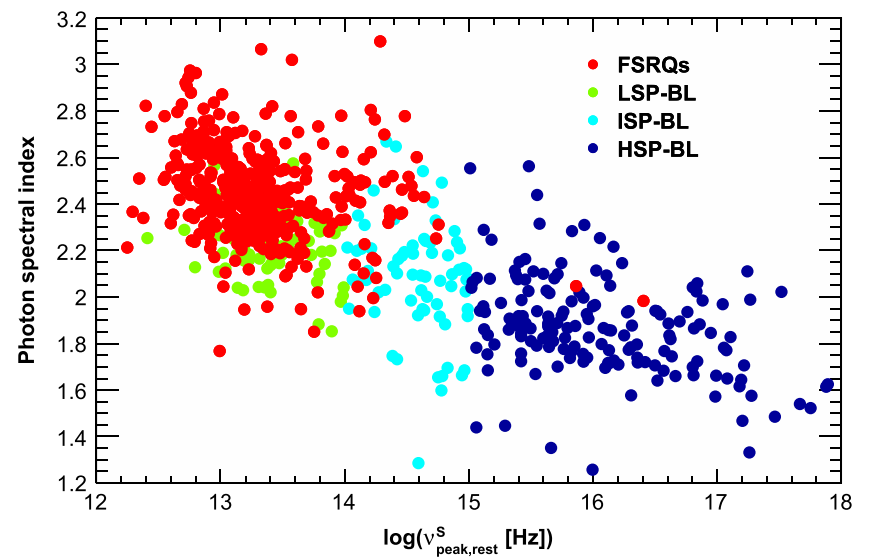

Figure 10. Photon index vs. frequency of the synchrotron peak $\nu_{\text {peak,rest }}^{\mathrm{S}} \cdot$ Red: FSRQs, green: LSP-BL Lacs, light blue: ISP-BL Lacs, dark blue: HSPBL Lacs.

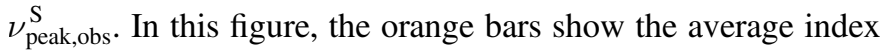
for different bins in $\nu_{\text {peak,rest }}^{\mathrm{S}}$ obtained from the data plotted in Figure 10 for blazars of known types. This comparison supports the idea that BCUs with low $\nu_{\text {peak }}^{\mathrm{S}}$ and high $\Gamma$ are likely FSRQs, while the rest would mostly be BL Lacs. ${ }^{86}$

\subsection{Redshift}

Figure 12 compares the redshift distributions for FSRQs and BL Lacs in the 2LAC Clean Sample and those for the new 3LAC Clean-Sample sources (note that $50 \%$ of the BL Lacs do not have measured redshifts; see below). The distributions are fairly similar, although the newly detected FSRQs are located at a slightly higher redshift than the $2 \mathrm{LAC}$ ones $(\langle z\rangle=1.33 \pm$ 0.08 versus $1.17 \pm 0.03)$. The maximum redshift for an FSRQ is still 3.1 (four FSRQs have $2.94<z<3.1$ ) and has not changed since the 1LAC. This trend allowed the conclusion that the number density of FSRQs grows dramatically up to redshift $\simeq 0.5-2.0$ and declines thereafter (Ajello et al. 2012).

The redshift distribution of new BL Lacs is somewhat narrower than that of the 2LAC sources, with a maximum near $z=0.3$. The redshift distributions gradually spread out to higher redshifts when moving from HSP-BL Lacs to LSP-BL Lacs, a feature already seen in 2LAC. However, the HSP distribution extends to higher redshifts relative to 2LAC, with six HSP-BL Lacs having measured redshifts greater than 1 and one (MG4 J000800+4712) having a redshift greater than 2 . Five of these six HSPs were already included in 2LAC but either lacked measured redshifts or were classified differently.

Among BL Lacs, 309 have a measured redshift, while 295 do not. The fraction of BL Lacs without redshift is $55 \%, 61 \%$, and $40 \%$ for LSPs, ISPs, and HSPs, respectively. However, Shaw et al. (2013) have provided redshift constraints for 134 2LAC BL Lacs: upper limits from the absence of Ly $\alpha$ absorption for all of them and lower limits from non-detection of the host galaxy or from intervening absorption line systems for a subset of 54 objects. It was noted by these authors that the average lower limit exceeded the average measured redshift for BL Lacs, indicating that the measured redshifts are biased low. The allowed redshift ranges for the 54 sources with both lower

\footnotetext{
${ }^{86}$ Note that Figure 11 plots $\nu_{\text {peak,obs }}^{\mathrm{S}}$ while Figure 10 shows $\nu_{\text {peak,rest }}^{\mathrm{S}}$. The $(1+$ z) correction, not applied in the latter case, is not expected to have a significant effect on the correlation strength.
}

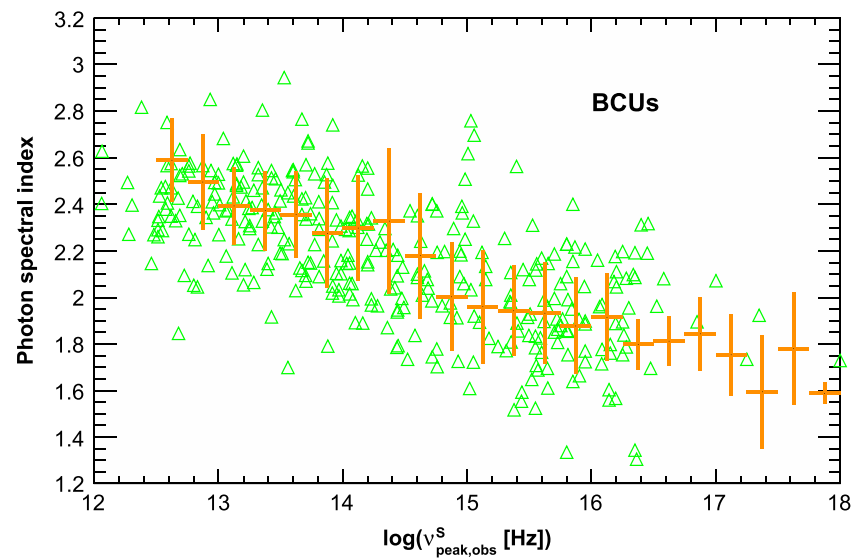

Figure 11. Photon index vs. frequency of the synchrotron peak $\nu_{\text {peak,obs }}^{\mathrm{S}}$ for blazars of unknown types (BCUs). For comparison, the orange bars show the average index for different bins in $\nu_{\text {peak,rest }}^{\mathrm{S}}$ for blazars with known types, as displayed in Figure 10.

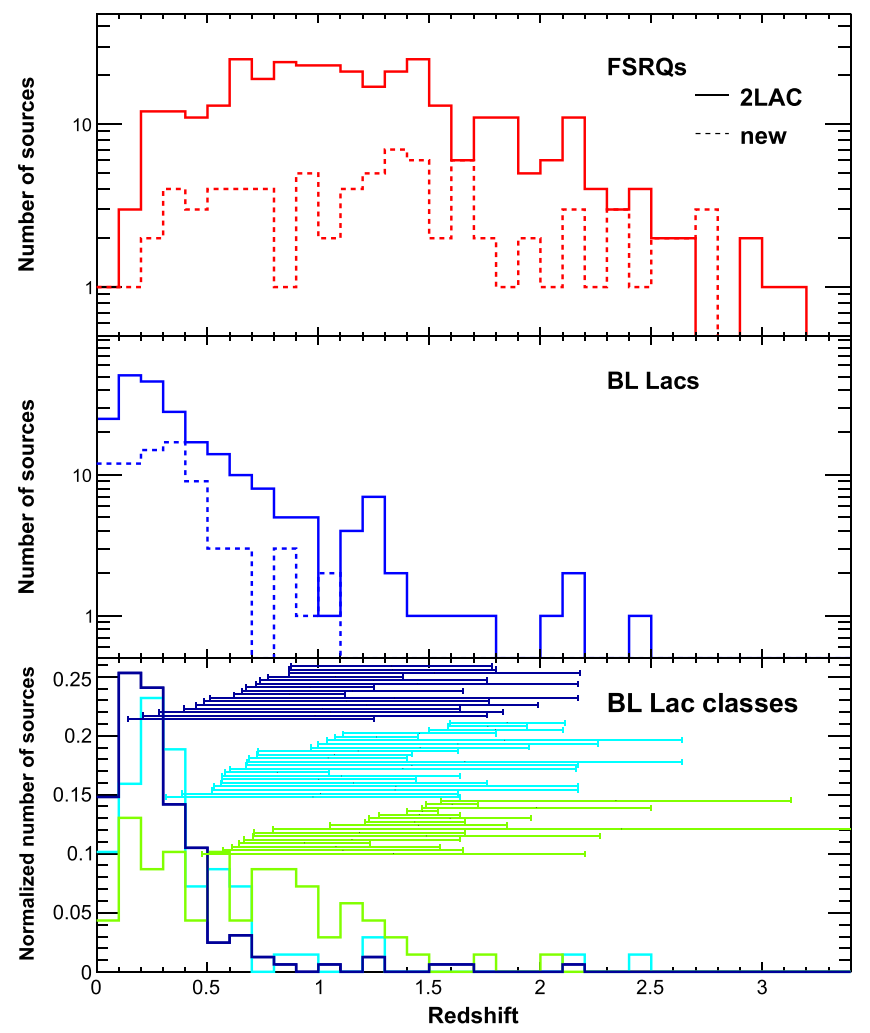

Figure 12. Redshift distributions (solid: 2LAC sources; dashed: new 3LAC sources) for FSRQs (top), BL Lacs (middle), and different types of BL Lacs (bottom): LSPs (green), ISPs (light blue), and HSPs (dark blue). The ranges between the lower and upper limits are also depicted in the bottom panel when both limits are available.

and upper limits are plotted in the bottom panel of Figure 12, confirming that they are in tension with the measured redshift distributions, in particular for HSPs. Kolmogorov-Smirnov tests $(\mathrm{K}-\mathrm{S})$ yield probabilities of $2 \times 10^{-2}, 1 \times 10^{-7}$, and $1 \times 10^{-6}$ that the distributions of measured redshifts and lower limits are drawn from the same underlying population for LSPs, ISPs and HSPs, respectively. The redshift ranges are very similar for the different subclasses and all cluster at high redshifts, with a median around $z=1.2$. This is in good agreement with the predictions of Giommi et al. (2013), which 


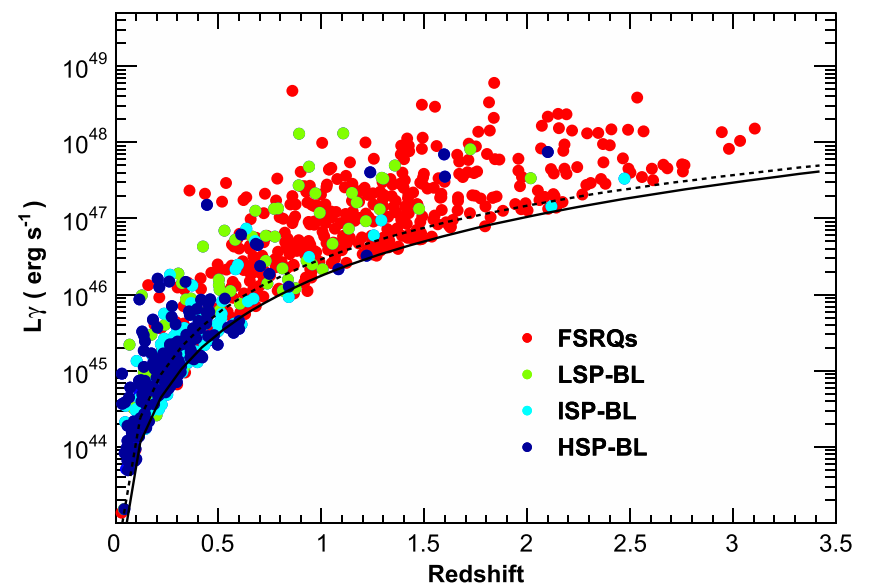

Figure 13. Gamma-ray luminosity vs. redshift. Red: FSRQs; green: LSP-BL Lacs; light blue: ISP-BL Lacs; dark blue: HSP-BL Lacs. The solid (dashed) curve represents the approximate detection limit for $\Gamma=1.8(\Gamma=2.2)$.

posit that most LAT-detected BL Lacs are actually FSRQs with their emission lines swamped by the non-thermal continuum hampering determination of their redshifts.

\subsection{Luminosity}

The gamma-ray luminosity has been computed from the 3FGL energy flux between $100 \mathrm{MeV}$ and $100 \mathrm{GeV}$, obtained by spectral fitting. Figure 13 displays the gamma-ray luminosity plotted against redshift, together with the sensitivity limits calculated for $\Gamma=1.8$ and 2.2. The Malmquist bias already reported in previous catalog papers is clearly visible. Low-luminosity BL Lacs $\left(<10^{45} \mathrm{erg} \mathrm{s}^{-1}\right)$ cannot be detected at $z>0.4$. Note that sources with a luminosity greater than $5 \times 10^{47} \mathrm{erg} \mathrm{s}^{-1}(64$ are in 3LAC) could still be detected at $z>3.2$.

Figure 14 shows the LAT photon index versus the gammaray luminosity for the different blazar classes. This correlation has been widely discussed in the context of the "blazar divide" or "blazar sequence" (Ghisellini et al. 2009, 2012; Meyer et al. 2012; Padovani et al. 2012; Finke 2013; Giommi et al. 2013). The features are similar to 2LAC, namely a branch of MAGNs separate from the bulk of blazars and a correlated trend of both luminosity and photon index as $\nu_{\text {peak }}^{\mathrm{S}}$ decreases. Figure 15 shows the LAT photon index versus the gamma-ray luminosity for the $57 \mathrm{BL}$ Lacs with both lower and upper limits on their redshifts or only upper limits (134 sources). Because of the bias mentioned above, the HSPs with both limits are more luminous on average than those with measured redshifts, thus populating a previously scarcely occupied area in the $L_{\gamma}-\Gamma$ diagram. This observation has profound consequences for the blazar sequence. Note that Ajello et al. (2014) found a small but significant correlation between gamma-ray luminosity and spectral index when including the redshift constraints from Shaw et al. (2013).

\subsection{Spectral Curvature}

First observed for 3C 454.3 (Abdo et al. 2009b) early in the Fermi mission, a significant curvature in the energy spectra of many bright FSRQs and some bright LSP-/ISP-BL Lacs is now a well-established feature (Abdo et al. 2010f, 2010g). The break energy obtained from a broken power-law fit has been found to be remarkably constant as a function of flux, at least

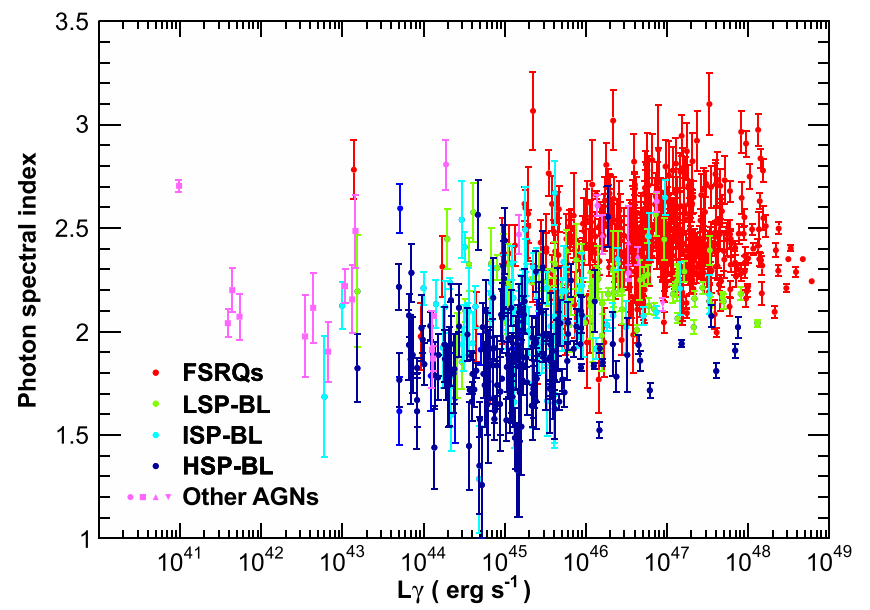

Figure 14. Photon index vs. gamma-ray luminosity. Red: FSRQs; green: LSPBL Lacs; light blue: ISP-BL Lacs; dark blue: HSP-BL Lacs; magenta: other AGNs (circles: NLSy1s; squares: radio galaxies; up triangles: SSRQs; down triangles: AGNs of other types).

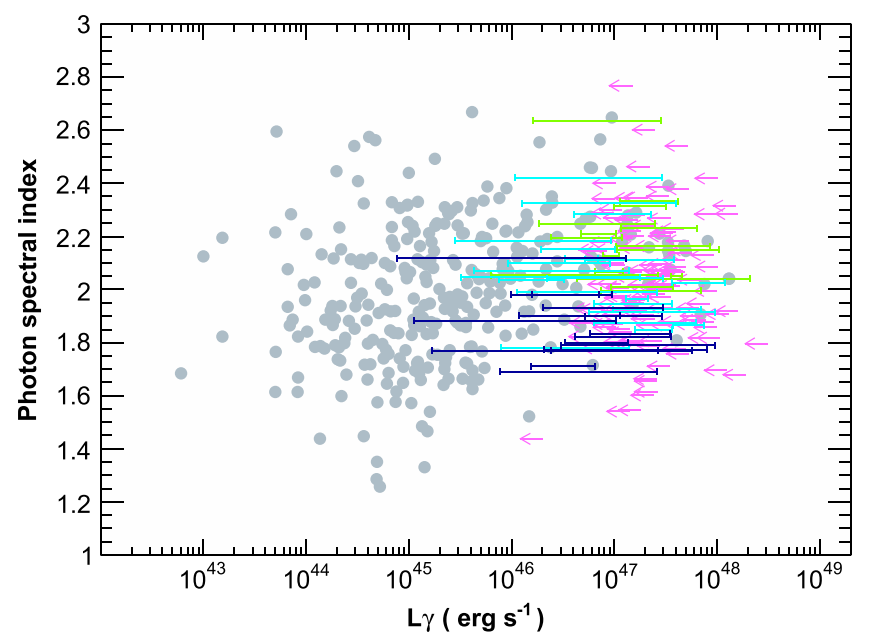

Figure 15. Photon index vs. gamma-ray luminosity for BL Lacs. Segments are plotted for sources having both lower- and upper-limits on their redshifts. Green: LSP-BL Lacs; light blue: ISP-BL Lacs; dark blue: HSP-BL Lacs. Magenta arrows are used for sources with upper limits only. BL Lacs with measured redshifts are depicted in gray, regardless of their SED classes.

for 3C 454.3 (Abdo et al. 2011). Several explanations have been proposed to account for this feature, including $\gamma \gamma$ attenuation from He II line photons (Poutanen \& Stern 2010), intrinsic electron spectral breaks (Abdo et al. 2009b), Ly $\alpha$ scattering (Ackermann et al. 2010), Klein-Nishina effects taking place when jet electrons scatter BLR radiation in a nearequipartition approach (Cerruti et al. 2013), and hybrid scattering (Finke \& Dermer 2010). The level of curvature has been observed to diminish during some flares (e.g., Pacciani et al. 2014).

In the 3FGL analysis, a switch is made from a power-law model to a log-parabola model whenever $\mathrm{TS}_{\text {curve }}>16$. The spectrum of the FSRQ 3C 454.3 cannot be well fitted with a log-parabola model, a power-law+exponential cutoff being a better model. A total of 91 FSRQs (57 in 2LAC), 32 BL Lacs (12 in 2LAC) and 8 BCUs show significant curvature at a confidence level $>99 \%$. Figure 16 shows the $\log$-parabola $\beta$ parameter plotted against gamma-ray flux and luminosity. At a given flux or luminosity the spectra of BL Lacs are less curved 

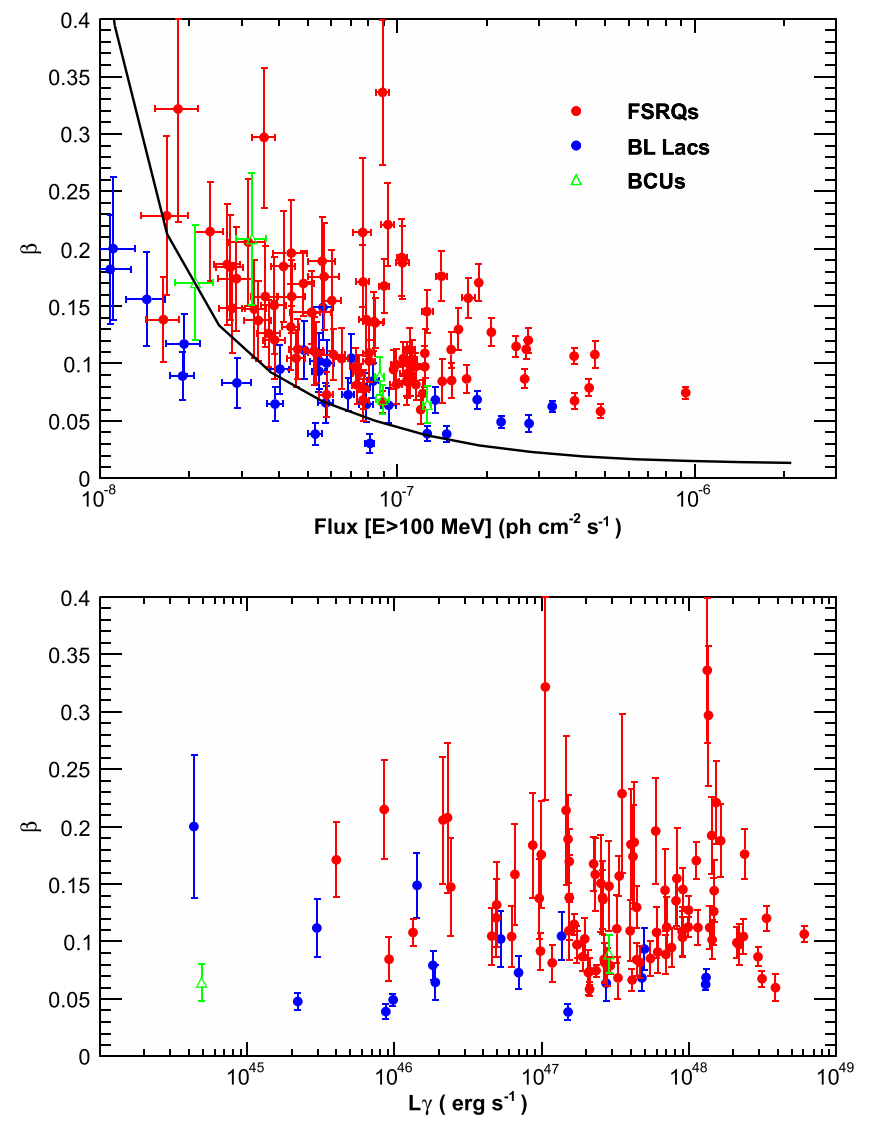

Figure 16. Log-parabola parameter $\beta$ plotted vs. photon flux above $100 \mathrm{MeV}$ (top, the line depicts the analysis limit $\mathrm{TS}_{\text {curve }}=16$ estimated for FSRQs) and gamma-ray luminosity (bottom). Red circles: FSRQs; blue circles: BL Lacs; green triangles: AGNs of unknown type.

than those of FSRQs, a feature already reported in 2LAC. Figure 17 compares the TS distributions for sources with curved spectra and those for the whole samples of FSRQs and BL Lacs. All bright FSRQs have curved spectra. For BL Lacs, the situation is more diverse. For BL Lac sources with TS $>1000$, the fraction of sources with curved spectra is 16/ $23(70 \%)$ for LSPs, 6/19 (32\%) for ISPs, and 5/28 (18\%) for HSPs. Note that because the latter have harder spectra than LSPs/ISPs on the average, potential spectral curvature is easier to detect for them. The average $\beta$ is lower for HSPs $(0.05)$ than for LSPs and ISPs (0.08).

\subsection{Variability}

Variability is a key feature of blazars. The 3FGL monthly averaged light curves provide a baseline reference against which other analyses can be cross-checked and enable crosscorrelation studies with data obtained at other wavelengths. Although variability at essentially all timescales has been observed in blazars, the monthly binning represents a trade off between a shorter binning needed to resolve flares in bright sources and a longer binning required to detect faint sources. Even so, only 15 sources are detected in all 48 bins with monthly significance $\mathrm{TS}>25$, while this number becomes 46 if a relaxed condition TS $>4$ is required. The 15 sources include $11 \mathrm{BL}$ Lacs (7 HSPs), only 3 FSRQs (PKS 1510-08, 4C +55.17, B2 1520+31) and the radio galaxy NGC 1275. The 46 sources comprise 28 BL Lacs (14 HSPs),

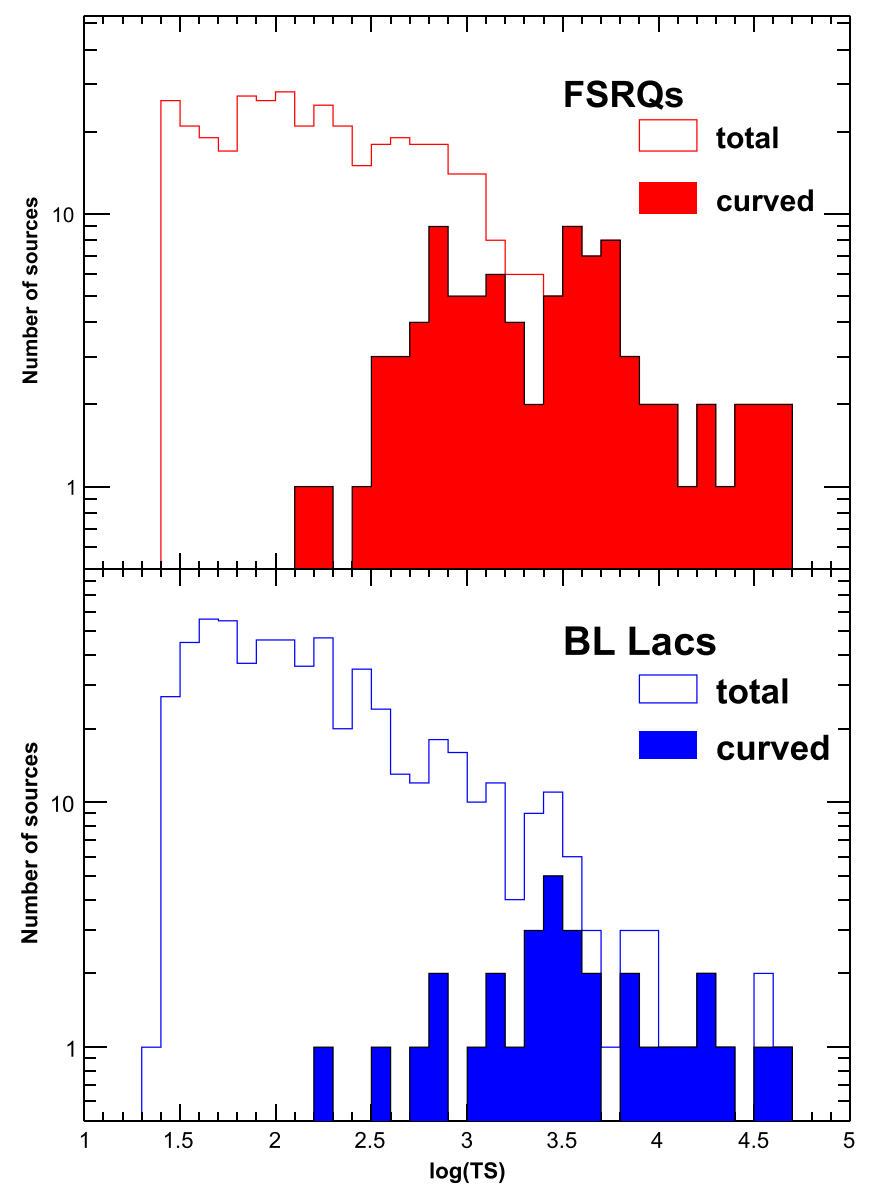

Figure 17. TS distributions of FSRQs (top) and BL Lacs (bottom). Solid histograms: total; filled histograms: sources with significant spectral curvatures.

15 FSRQs, one BCU and two radio galaxies, NGC 1275 and Centaurus A.

We will focus here on the variability index defined in Section 2; a value of 72.44 for this index indicating variability at the $99 \%$ confidence level (while the average index for nonvariable sources is 47). Recall that this index can be large only for sources that are both variable and relatively bright. This index is plotted versus the synchrotron peak frequency in Figure 18. The features already reported in $2 \mathrm{LAC}$ are again visible, with a large fraction of FSRQs found to be variable $(69 \%)$, with the fraction for BL Lacs much lower on average $(23 \%)$ and with a steadily decreasing trend as $\nu_{\text {peak }}^{\mathrm{S}}$ rises $(39 \%$, $23 \%, 15 \%$ for LSPs, ISPs and HSPs respectively). These fractions are quite similar to those reported in 2LAC, despite the larger population and longer time span of the light curves. A similar trend between variability index and $\nu_{\text {peak }}^{\mathrm{S}}$ is observed for BCU (Figure 18 bottom), with $21 \%$ of them found to be variable.

The variability index is plotted versus TS for different bins in the photon spectral index in Figure 19. A distinct trend is visible: for a given TS the mean variability index increases as the spectrum becomes softer (the spectral index increases) up to $\Gamma=2.4$ where this effect saturates. A net difference between FSRQs and BL Lacs is also apparent, confirming the behavior reported above. For $\Gamma>2.2,72 \%$ of FSRQs and $25 \%$ of BL Lacs are variable above the $99 \%$ confidence level. 

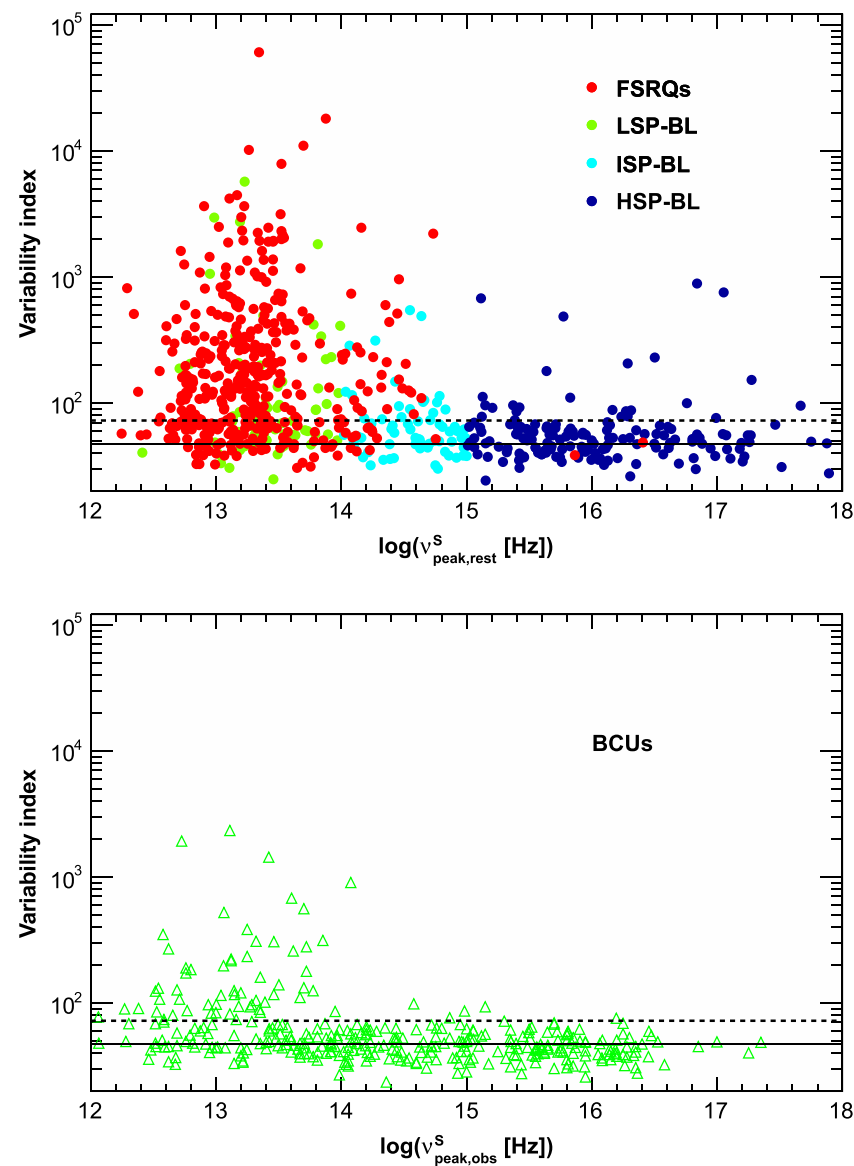

Figure 18. Top: variability index vs. rest-frame synchrotron peak frequency. Red: FSRQs; green: LSP-BL Lacs; light blue: ISP-BL Lacs; dark blue: HSPBL Lacs. The solid line depicts the average variability index expected for nonvariable sources. The dashed line corresponds to the $99 \%$ confidence level for a source to be variable. Bottom: variability index vs. observed synchrotron peak frequency for BCUs. The lines are the same as in the upper panel.

For each source, we fit the distribution of monthly photon fluxes with a lognormal function

$$
f_{\mathrm{Ln}}(x)=\frac{N_{\mathrm{Ln}}}{x \sigma_{\mathrm{Ln}} \sqrt{2 \pi}} \exp \left[-\frac{\left(\log (x)-u_{\mathrm{Ln}}\right)^{2}}{2 \sigma_{\mathrm{Ln}}^{2}}\right],
$$

treating the flux values returned by the maximum-likelihood algorithm as if they were always significant, for simplicity. The lognormal function has commonly been used to model blazar flux distributions (e.g., Giebels \& Degrange 2009; Tluczykont et al. 2010) and provides reasonable fits for most sources of our large sample. This distribution is expected for a process involving a large number of multiplicative, independently varying parameters. Figure 20 compares the distributions of shape parameters $\sigma_{\mathrm{Ln}}$ of FSRQs and BL Lacs that have been detected in 48 months above a TS of 1000 and had a monthly TS above 4 in at least 24 monthly periods. These distributions are distinct. The modes are about 0.8 and 0.4 for FSRQs and BL Lacs, respectively, confirming a larger flux variability for the former.

To further illustrate the detection variability and how the sample of brightest blazars renews itself, we compare the samples of brightest sources detected during the first and the last three-month periods of the 4 year-long data accumulation

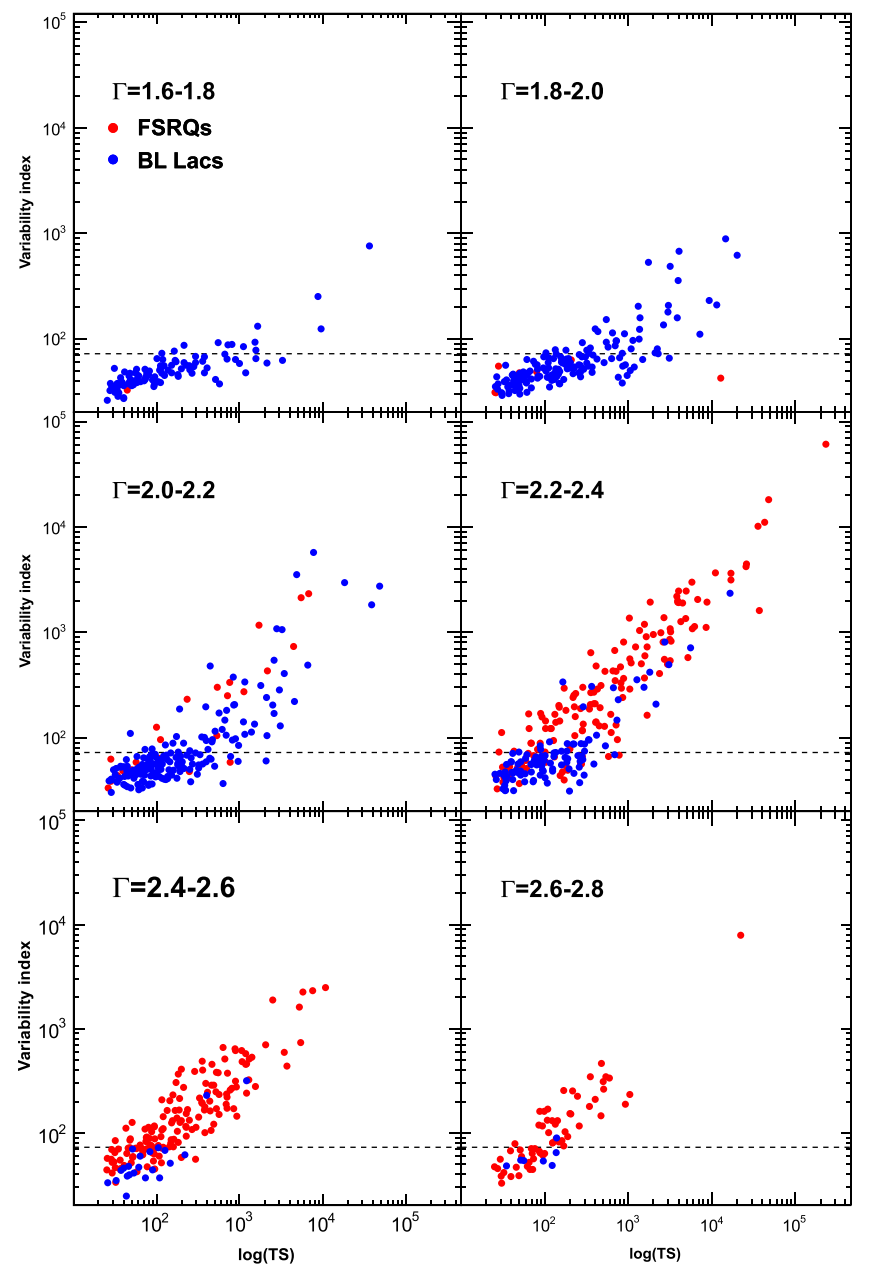

Figure 19. Top: variability index vs. TS for 6 bins in the photon spectral index $\Gamma$. Red: FSRQs; blue: BL Lacs. The dashed line corresponds to the $99 \%$ confidence level for a source to be variable.

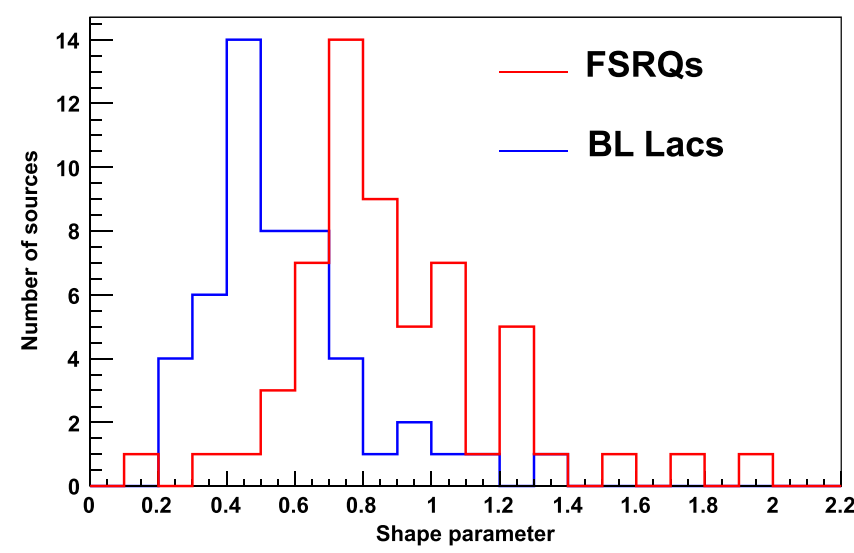

Figure 20. Log-normal-function shape parameters $\sigma_{\mathrm{Ln}}$ obtained from the monthly flux distributions of TS $>1000$ FSRQs (red) and BL Lacs (blue).

time. We applied the same TS cut used to select the LBAS sample (Abdo et al. 2009a), namely TS $>100$ (simply adding up the monthly TS values). The two samples include similar numbers of sources (128 versus 134), but have only $50 \%$ (65) of the sources in common. 
Table 8

3LAC Sources: Fluxes (High-latitude Sources)

\begin{tabular}{lccccccc}
\hline \hline $\begin{array}{l}\text { 3FGL Source } \\
\begin{array}{l}\text { Name } \\
(1)\end{array}\end{array}$ & $\begin{array}{c}\text { Counterpart } \\
\text { Name } \\
(2)\end{array}$ & $\begin{array}{c}\text { Radio Flux } \\
(\mathrm{mJy})\end{array}$ & Radio Flag & $\begin{array}{c}\text { X-ray Flux } \\
(3)\end{array}$ & $(4)$ & $\begin{array}{c}\text { USNO B1 } \\
V \text { mag } \\
(6)\end{array}$ & $\begin{array}{c}\text { SDSS } \\
V \text { mag } \\
(7)\end{array}$ \\
\hline J0001.2-0748 & PMN J0001-0746 & 209.17 & $\mathrm{~N}$ & 8.100 & 17.612 & $\begin{array}{c}\alpha_{\text {ox }} \\
(8)\end{array}$ & $\begin{array}{c}\alpha_{\text {ro }} \\
(9)\end{array}$ \\
\hline
\end{tabular}

Notes. Column 1 is the 3FGL name, column 2 is the candidate counterpart name, column 3 is the radio flux measured in the survey indicated in column 4: N for NVSS $(1.4 \mathrm{GHz}), \mathrm{S}$ for SUMSS $(845 \mathrm{MHz})$, A for ATCA $(20 \mathrm{GHz}), \mathrm{P}$ indicates PMN $(4.8 \mathrm{GHz})$, and F indicates FIRST at $1.4 \mathrm{GHz}$. Column 5 is the X-ray flux between 0.1 and $2.4 \mathrm{keV}$ from the RASS survey (Voges et al. 1999, 2000), columns 6-7 shows the USNO and SDSS $V$ magnitudes, respectively. Columns 8 and 9 show the broadband indices between $5000 \AA$ and $1 \mathrm{keV}\left(\alpha_{\mathrm{ox}}\right)$ and between $5 \mathrm{GHz}$ and $5000 \AA\left(\alpha_{\mathrm{ro}}\right)$.

${ }^{a}$ Refers to sources in the Clean Sample.

(This table is available in its entirety in machine-readable form.)

\section{MULTIWAVELENGTH PROPERTIES OF 3LAC SOURCES}

It was shown in 2LAC that the LAT-detected blazars display on average larger radio fluxes than non-detected blazars and that they are all bright in the optical. Tables 8 and 9 give archival data for the 3LAC and low-latitude sources, respectively. Below we focus on the connection with the two neighboring bands, namely the hard X-rays and the VHE bands.

\subsection{Sources Detected in Hard X-Rays}

A total of 85 3LAC sources are in common with the Swift BAT 70 months survey (Baumgartner et al. 2013) in the 14-195 keV band performed between December 2004 and September 2010 (there were 47 in 2LAC). These 85 sources include 34 FSRQs with an average redshift of $1.37 \pm 0.15$. Only 9 BAT FSRQs are missing from 3LAC. The average LAT photon index of BAT-detected FSRQs is 2.57, i.e., somewhat softer than the overall average photon index of LAT FSRQs (2.43), a clue that their high-energy hump is located at slightly lower energies than the bulk of the FSRQs. Out of 37 BAT BL Lacs, 30 have now been detected with the LAT. These BL Lacs comprise 3 LSPs, 2 ISPs, and 19 HSPs, while 4 others are still unclassified. The large fraction of HSPs in this sample is not surprising, as the detection of LSPs and ISPs in the hard X-ray band is hampered by their SEDs exhibiting a valley between the low- and high-energy humps in this band (see Böttcher 2007). Figure 21 displays the LAT photon index versus the BAT photon index. Despite large error bars in the BAT photon index and non-simultaneous measurements, a remarkable anticorrelation (Pearson correlation factor -0.69), already noted in 2LAC, is observed. For the HSP-BL Lacs considered here, BAT probes the high-frequency (falling) part of the $\nu F_{\nu}$ synchrotron peak while the LAT probes the rising side of the inverse-Compton peak (assuming a leptonic scenario). For FSRQs, which are all LSPs in the common sample, BAT and LAT probe the rising and falling sides of the inverse-Compton peak, respectively.

It is also worth noting that 96 3LAC sources (5 Radio Galaxies, 53 FSRQs, 33 BL Lacs, 4 BCUs, 1 NLSy1) are present in the V38 INTEGRAL source catalog ${ }^{87}$ (based on 3-200 keV data taken since 2002), which includes 540 AGNs located at $|b|>10^{\circ}$.

\footnotetext{
${ }^{87}$ http://www.isdc.unige.ch/integral/science/catalogue
}

\subsection{Sources Detected at Very High Energies}

At the time of this writing, 56 AGNs that have been detected at $\mathrm{TeV}$ energies are listed in TeVCat. ${ }^{88}$ Among them, 55 are present in 3FGL (see Table 10), which is a remarkable result underscoring the level of synergy that has now been achieved between the high-energy and VHE domains. Only HESS $\mathrm{J} 1943+213$ ( $a$ HSP BL Lac located at $b=-1.3$, affecting the possible LAT detection) is still missing from the 3FGL, but an analysis of five years of LAT data resulted in a $>1 \mathrm{GeV}$ detection (Peter et al. 2014). There are 15 newly detected sources relative to 2FGL and six relative to the first Fermi-LAT catalog of sources above $10 \mathrm{GeV}$ (1FHL, Ackermann et al. 2013, based on 3 years of data): SHBL J001355.9 -185406, 1ES 0229+200, 1ES 0347-121, RX J0847.1+1133 (aka RBS 0723), MS 1221.8+2452, and 1 H 1720+117.

Not all of the 55 sources are included in the 3LAC Clean Sample, either because they are located at low Galactic latitudes or because they are flagged for different reasons. The average photon index for HSP BL Lacs (representing 39 of the $55 \mathrm{AGNs}$ ) is $1.78 \pm 0.13$ (rms), slightly harder than that for the whole 3 LAC sample $(1.88 \pm 0.22)$. Only 28 out of the 55 3FGL sources are seen to be variable in the LAT energy range at a significance greater than $99 \%$.

\section{DISCUSSION}

\subsection{Gamma-Ray Detected versus Non-detected Blazars}

The blazars detected in gamma-rays after 4 years of LAT operation represent a sizeable fraction of the whole population of known blazars as listed in BZCAT. BZCAT represents an exhaustive list of sources ever classified as blazars but is by no means complete. Although a comparison between the gammaray detected and non-detected blazars within that sample has no strong statistical meaning in terms of relative weights, it is nevertheless useful to look for general trends.

The overall LAT-detected fraction is $24 \%$ (409/1707) for FSRQs, 44\% (543/1221) for BL Lacs and 27\% (59/221) for BCUs. A comparison between the normalized redshift distributions of the BZCAT blazars either included or not included in 3LAC is given in Figure 22, as well as the fraction of 3LAC sources relative to the total for a given redshift. A K$\mathrm{S}$ test gives a probability of $3 \times 10^{-8}$ that the two redshift distributions are drawn from the same population. The distribution shapes are quite similar for the two subsets

\footnotetext{
${ }^{88}$ http://tevcat.uchicago.edu
} 
Table 9

3LAC Sources: Fluxes (Low-latitude Sources)

\begin{tabular}{|c|c|c|c|c|}
\hline $\begin{array}{c}\text { Name } \\
(1)\end{array}$ & $\begin{array}{l}\text { Name } \\
\text { (2) }\end{array}$ & $\begin{array}{c}(\mathrm{mJy}) \\
(3)\end{array}$ & $\begin{array}{l}\text { Radio } \\
\text { Flag }\end{array}$ & $\begin{array}{c}\text { X-ray } \\
\text { Flux } \\
\left(10^{-13} \text { erg }\right. \\
\left.\mathrm{cm}^{-2} \mathrm{~s}^{-1}\right) \\
(5)\end{array}$ \\
\hline $\mathrm{J} 0012.4+7040$ & TXS $0008+704$ & 639 & $\mathrm{~N}$ & $\cdots$ \\
\hline J0014.6+6119 & $4 C+60.01$ & 4040 & $\mathrm{~N}$ & $\ldots$ \\
\hline J0014.7+5802 & $\begin{array}{l}\text { 1RXS J001442.2 } \\
+580201\end{array}$ & 7.7 & $\mathrm{~N}$ & 104 \\
\hline $\mathrm{J} 0015.7+5552$ & GB6 J0015+5551 & 85 & $\mathrm{~N}$ & 152 \\
\hline J0035.9+5949 & 1ES 0033+595 & 148 & $\mathrm{~N}$ & 318 \\
\hline $\mathrm{J} 0047.0+5658$ & GB6 J0047+5657 & 190 & $\mathrm{~N}$ & $\cdots$ \\
\hline J0047.9+5447 & $\begin{array}{l}\text { 1RXS J004754.5 } \\
\quad+544758\end{array}$ & 13.9 & $\mathrm{~N}$ & 31.2 \\
\hline $\mathrm{J} 0102.8+5825$ & TXS 0059+581 & 849 & $\mathrm{~N}$ & $\ldots$ \\
\hline J0103.4+5336 & $\begin{array}{l}\text { 1RXS J010325.9 } \\
\quad+533721\end{array}$ & 30.9 & $\mathrm{~N}$ & 63.7 \\
\hline J0109.8+6132 & TXS 0106+612 & 305 & $\mathrm{~N}$ & $\cdots$ \\
\hline J0110.2+6806 & $4 C+67.04$ & 1715 & $\mathrm{~N}$ & 23.2 \\
\hline $\mathrm{J} 0131.2+6120$ & $\begin{array}{l}\text { 1RXS J013106.4 } \\
\quad+612035\end{array}$ & 19.7 & $\mathrm{~N}$ & 471 \\
\hline $\mathrm{J} 0131.3+5548$ & TXS 0128+554 & 175 & $\mathrm{~N}$ & 21.9 \\
\hline J0135.0+6927 & TXS 0130+691 & 202 & $\mathrm{~N}$ & $\cdots$ \\
\hline J0137.8+5813 & $\begin{array}{l}\text { 1RXS J013748.0 } \\
\quad+581422\end{array}$ & 171 & $\mathrm{~N}$ & 252 \\
\hline J0148.3+5200 & GB6 J0148+5202 & 44.5 & $\mathrm{~N}$ & $\cdots$ \\
\hline J0153.4+7114 & TXS 0149+710 & 578 & $\mathrm{~N}$ & 48.3 \\
\hline $\mathrm{J} 0211.7+5402$ & TXS $0207+538$ & 448 & $\mathrm{~N}$ & $\ldots$ \\
\hline J0214.4+5143 & TXS $0210+515$ & 295 & $\mathrm{~N}$ & 177 \\
\hline J0217.3+6209 & TXS $0213+619$ & 155 & $\mathrm{~N}$ & $\cdots$ \\
\hline J0223.3+6820 & $\begin{array}{c}\text { NVSS J022304 } \\
+682154\end{array}$ & 20 & $\mathrm{~N}$ & $\cdots$ \\
\hline $\mathrm{J} 0223.5+6313$ & TXS 0219+628 & 124 & $\mathrm{~N}$ & $\cdots$ \\
\hline $\mathrm{J} 0228.5+6703$ & GB6 J0229+6706 & 27 & $\mathrm{~N}$ & $\cdots$ \\
\hline $\mathrm{J} 0241.3+6542$ & TXS 0237+655 & 191 & $\mathrm{~N}$ & 41.6 \\
\hline $\mathrm{J} 0250.6+5630$ & $\begin{array}{l}\text { NVSS J025047 } \\
+562935\end{array}$ & 35.8 & $\mathrm{~N}$ & 34.3 \\
\hline J0253.8+5104 & $\begin{array}{l}\text { NVSS J025357 } \\
\quad+510256\end{array}$ & 429 & $\mathrm{~N}$ & $\cdots$ \\
\hline $\mathrm{J} 0302.0+5335$ & GB6 J0302+5331 & 187 & $\mathrm{~N}$ & $\cdots$ \\
\hline J0303.6+4716 & $4 C+47.08$ & 963 & $\mathrm{~N}$ & $\cdots$ \\
\hline J0304.9+6817 & TXS 0259+681 & 1208 & $\mathrm{~N}$ & $\cdots$ \\
\hline $\mathrm{J} 0332.0+6308$ & GB6 J0331+6307 & 42.8 & $\mathrm{~N}$ & $\cdots$ \\
\hline J0333.9+6538 & TXS 0329+654 & 288 & $\mathrm{~N}$ & 16.6 \\
\hline $\mathrm{J} 0352.9+5655$ & GB6 J0353+5654 & 58.3 & $\mathrm{~N}$ & $\cdots$ \\
\hline $\mathrm{J} 0354.1+4643$ & B3 0350+465 & 759 & $\mathrm{~N}$ & $\cdots$ \\
\hline $\mathrm{J} 0358.8+6002$ & TXS 0354+599 & 953 & $\mathrm{~N}$ & 38.8 \\
\hline J0418.5+3813 & 3C 111 & 7731 & $\mathrm{~N}$ & 142 \\
\hline $\mathrm{J} 0423.8+4150$ & $4 \mathrm{C}+41.11$ & 1756 & $\mathrm{~N}$ & $\cdots$ \\
\hline $\mathrm{J} 0425.2+6319$ & $\begin{array}{l}\text { 1RXS J042523.0 } \\
+632016\end{array}$ & 25.2 & $\mathrm{~N}$ & 44.3 \\
\hline $\mathrm{J} 0444.5+3425$ & B2 $0441+34$ & 238 & $\mathrm{~N}$ & $\cdots$ \\
\hline $\mathrm{J} 0501.8+3046$ & $\begin{array}{c}\text { 1RXS J050140.8 } \\
+304831\end{array}$ & 35.2 & $\mathrm{~N}$ & 62.7 \\
\hline J0502.7+3438 & MG2 J050234+3436 & 176 & $\mathrm{~N}$ & $\cdots$ \\
\hline J0503.4+4522 & $\begin{array}{c}\text { 1RXS J050339.8 } \\
+451715\end{array}$ & 34.9 & $\mathrm{~N}$ & 75.2 \\
\hline J0512.2+2918 & B2 0509+29 & 204 & $\mathrm{~N}$ & 19.5 \\
\hline J0512.9+4038 & B3 0509+406 & 877 & $\mathrm{~N}$ & $\cdots$ \\
\hline J0517.4+4540 & $4 C+45.08$ & 1336 & $\mathrm{~N}$ & $\cdots$ \\
\hline J0519.3+2746 & $4 \mathrm{C}+27.15$ & 1702 & $\mathrm{~N}$ & $\cdots$ \\
\hline $\mathrm{J} 0521.7+2113$ & TXS $0518+211$ & 530 & $\mathrm{~N}$ & 60.2 \\
\hline $\mathrm{J} 0526.0+4253$ & $\begin{array}{l}\text { NVSS J052520 } \\
\quad+425520\end{array}$ & 41.6 & $\mathrm{~N}$ & $\cdots$ \\
\hline J0528.3+1815 & & 21.5 & $\mathrm{~N}$ & 163 \\
\hline
\end{tabular}

Table 9

(Continued)

\begin{tabular}{|c|c|c|c|c|}
\hline 3FGL Source & Counterpart & $\begin{array}{l}\text { Radio } \\
\text { Flux }\end{array}$ & $\begin{array}{l}\text { Radio } \\
\text { Flag }\end{array}$ & $\begin{array}{c}\text { X-ray } \\
\text { Flux } \\
\left(10^{-13} \text { erg }\right.\end{array}$ \\
\hline Name & Name & $(\mathrm{mJy})$ & & $\left.\mathrm{cm}^{-2} \mathrm{~s}^{-1}\right)$ \\
\hline$(1)$ & $(2)$ & (3) & (4) & (5) \\
\hline & $\begin{array}{l}\text { 1RXS J052829.6 } \\
+181657\end{array}$ & & & \\
\hline J0533.2+4822 & TXS 0529+483 & 435 & $\mathrm{~N}$ & 10.8 \\
\hline J0539.8+1434 & TXS 0536+145 & 433 & $\mathrm{~N}$ & $\cdots$ \\
\hline J0601.0+3837 & B2 $0557+38$ & 705 & $\mathrm{~N}$ & $\cdots$ \\
\hline J0603.8+2155 & $4 \mathrm{C}+22.12$ & 2772 & $\mathrm{~N}$ & $\ldots$ \\
\hline J0611.7+2759 & GB6 J0611+2803 & 22.2 & $\mathrm{~N}$ & $\ldots$ \\
\hline J0620.4+2644 & RX J0620.6+2644 & 82.6 & $\mathrm{~N}$ & 214 \\
\hline J0622.9+3326 & B2 0619+33 & 240 & $\mathrm{~N}$ & $\ldots$ \\
\hline J0623.3+3043 & GB6 J0623+3045 & 52.2 & $\mathrm{~N}$ & $\ldots$ \\
\hline J0631.2+2019 & TXS 0628+203 & 317 & $\mathrm{~N}$ & $\cdots$ \\
\hline J0640.0-1252 & TXS 0637-128 & 225 & $\mathrm{~N}$ & 312 \\
\hline J0641.8-0319 & TXS 0639-032 & 820 & $\mathrm{~N}$ & $\ldots$ \\
\hline J0643.2+0859 & PMN J0643+0857 & 543 & $\mathrm{~N}$ & $\ldots$ \\
\hline J0648.1+1606 & $\begin{array}{l}\text { 1RXS J064814.1 } \\
\quad+160708\end{array}$ & 25.0 & $\mathrm{~N}$ & 34.6 \\
\hline J0648.8+1516 & RX J0648.7+1516 & 64.8 & $\mathrm{~N}$ & 381 \\
\hline J0648.8-1740 & TXS 0646-176 & 1046 & $\mathrm{~N}$ & $\ldots$ \\
\hline J0650.4-1636 & PKS 0648-16 & 1778 & $\mathrm{~N}$ & $\ldots$ \\
\hline J0650.5+2055 & $\begin{array}{l}\text { 1RXS J065033.9 } \\
+205603\end{array}$ & 6.90 & $\mathrm{~N}$ & 18.2 \\
\hline J0654.5+0926 & RX J0654.3+0925 & 44.4 & $\mathrm{~N}$ & 50.3 \\
\hline J0656.2-0323 & TXS 0653-033 & 403 & $\mathrm{~N}$ & $\cdots$ \\
\hline J0658.6+0636 & $\begin{array}{l}\text { NVSS J065844 } \\
\quad+063711\end{array}$ & 25 & $\mathrm{~N}$ & $\cdots$ \\
\hline J0700.0+1709 & TXS $0657+172$ & 648 & $\mathrm{~N}$ & $\cdots$ \\
\hline J0700.2+1304 & GB6 J0700+1304 & 78 & $\mathrm{~N}$ & $\cdots$ \\
\hline J0702.7-1952 & TXS 0700-197 & 527 & $\mathrm{~N}$ & $\cdots$ \\
\hline J0709.7-0256 & PMN J0709-0255 & 153 & $\mathrm{~N}$ & $\cdots$ \\
\hline J0721.4+0404 & PMN J0721+0406 & 313 & $\mathrm{~N}$ & 12.9 \\
\hline J0723.2-0728 & $\begin{array}{c}1 \mathrm{RXS} \\
\mathrm{J} 072259.5-073131\end{array}$ & 85 & $\mathrm{~N}$ & 150 \\
\hline J0725.8-0054 & PKS 0723-008 & 1400 & $\mathrm{~N}$ & $\ldots$ \\
\hline J0729.5-3127 & $\begin{array}{c}\text { NVSS } \\
\text { J072922-313128 }\end{array}$ & 38 & $\mathrm{~N}$ & $\cdots$ \\
\hline J0730.2-1141 & PKS 0727-11 & 2760 & $\mathrm{~N}$ & $\cdots$ \\
\hline J0730.5-0537 & TXS 0728-054 & 168 & $\mathrm{~N}$ & $\cdots$ \\
\hline J0744.1-3804 & PMN J0743-3804 & 223 & $\mathrm{~N}$ & $\cdots$ \\
\hline J0744.8-4028 & PMN J0744-4032 & 65 & A & $\cdots$ \\
\hline J0746.6-0706 & PMN J0746-0709 & 55 & $\mathrm{~N}$ & $\cdots$ \\
\hline J0747.2-3311 & PKS 0745-330 & 726 & $\mathrm{~N}$ & $\cdots$ \\
\hline J0748.0-1639 & TXS 0745-165 & 803 & $\mathrm{~N}$ & $\cdots$ \\
\hline J0754.4-1148 & TXS 0752-116 & 881 & $\mathrm{~N}$ & $\cdots$ \\
\hline J0804.0-3629 & $\begin{array}{c}\text { NVSS } \\
\text { J080405-362919 }\end{array}$ & 57 & $\mathrm{~N}$ & $\cdots$ \\
\hline J0816.7-2421 & PMN J0816-2421 & 191 & $\mathrm{~N}$ & $\ldots$ \\
\hline J0825.8-3217 & PKS 0823-321 & 393 & $\mathrm{~N}$ & $\ldots$ \\
\hline J0825.9-2230 & PKS 0823-223 & 520 & $\mathrm{~N}$ & 36.9 \\
\hline J0828.8-2420 & $\begin{array}{c}\text { NVSS } \\
\text { J082841-241850 }\end{array}$ & 249 & $\mathrm{~N}$ & $\ldots$ \\
\hline J0841.3-3554 & $\begin{array}{c}\text { NVSS } \\
\text { J084121-355506 }\end{array}$ & 74 & $\mathrm{~N}$ & $\cdots$ \\
\hline J0845.1-5458 & PMN J0845-5458 & 916 & A & 9.04 \\
\hline J0849.5-2912 & $\begin{array}{c}\text { NVSS } \\
\text { J084922-291149 }\end{array}$ & 21.3 & $\mathrm{~N}$ & $\cdots$ \\
\hline J0849.9-3540 & PMN J0849-3541 & 376 & $\mathrm{~N}$ & $\ldots$ \\
\hline J0852.6-5756 & PMN J0852-5755 & 403 & $\mathrm{~N}$ & 16.2 \\
\hline J0853.0-3654 & $\begin{array}{c}\text { NVSS } \\
\text { J085310-365820 }\end{array}$ & 206 & $\mathrm{~N}$ & $\ldots$ \\
\hline J0858.1-3130 & & 5.6 & $\mathrm{~N}$ & 111 \\
\hline
\end{tabular}


Table 9

(Continued)

\begin{tabular}{|c|c|c|c|c|}
\hline $\begin{array}{l}\text { Name } \\
(1)\end{array}$ & $\begin{array}{l}\text { Name } \\
(2)\end{array}$ & $\begin{array}{l}\text { Radio } \\
\text { Flux } \\
(\mathrm{mJy}) \\
(3)\end{array}$ & $\begin{array}{l}\text { Radio } \\
\text { Flag }\end{array}$ & $\begin{array}{c}\text { X-ray } \\
\text { Flux } \\
\left(10^{-13} \text { erg }\right. \\
\left.\mathrm{cm}^{-2} \mathrm{~s}^{-1}\right) \\
(5)\end{array}$ \\
\hline J0904.8-3516 & $\begin{array}{c}\text { 1RXS } \\
\text { J085802.6-313043 } \\
\text { NVSS } \\
\text { J090442-351423 }\end{array}$ & 279 & $\mathrm{~N}$ & $\cdots$ \\
\hline J0904.8-5734 & PKS 0903-57 & 1434 & A & $\cdots$ \\
\hline J0922.8-3959 & PKS 0920-39 & 2616 & $\mathrm{~N}$ & 16.6 \\
\hline J0940.7-6102 & MRC 0939-608 & 491 & A & $\cdots$ \\
\hline J0956.7-6441 & $\begin{array}{c}\text { AT20G } \\
\text { J095612-643928 }\end{array}$ & 70 & A & $\cdots$ \\
\hline J1005.0-4959 & PMN J1006-5018 & 1177 & A & $\ldots$ \\
\hline $\mathrm{J} 1015.2-4512$ & PMN J1014-4508 & 542 & A & $\cdots$ \\
\hline J1038.9-5311 & MRC 1036-529 & 1675 & A & $\cdots$ \\
\hline $\mathrm{J} 1047.8-6216$ & PMN J1047-6217 & 2285 & A & $\cdots$ \\
\hline J1051.5-6517 & PKS 1049-650 & 220 & A & $\ldots$ \\
\hline J1103.9-5357 & PKS 1101-536 & 539 & A & $\cdots$ \\
\hline J1123.2-6415 & $\begin{array}{c}\text { AT20G } \\
\text { J112319-641735 }\end{array}$ & 280 & A & $\cdots$ \\
\hline J1136.6-6826 & PKS 1133-681 & 585 & A & $\cdots$ \\
\hline J1229.8-5305 & $\begin{array}{c}\text { AT20G } \\
\text { J122939-530332 }\end{array}$ & 56 & A & $\cdots$ \\
\hline J1233.9-5736 & $\begin{array}{c}\text { AT20G } \\
\text { J123407-573552 }\end{array}$ & 59 & A & $\cdots$ \\
\hline J1256.1-5919 & PMN J1256-5919 & 72 & A & $\ldots$ \\
\hline J1304.3-5535 & PMN J1303-5540 & 905 & A & $\cdots$ \\
\hline J1308.1-6707 & PKS 1304-668 & 611 & A & $\ldots$ \\
\hline
\end{tabular}

Note. Column 1 is the 3FGL name, column 2 is the candidate counterpart name, column 3 is the radio flux measured in the survey indicated in column 4 : $\mathrm{N}$ for NVSS $(1.4 \mathrm{GHz}), \mathrm{S}$ for SUMSS $(845 \mathrm{MHz})$, A for ATCA $(20 \mathrm{GHz}), \mathrm{P}$ indicates PMN $(4.8 \mathrm{GHz})$, and F indicates FIRST at $1.4 \mathrm{GHz}$. Column 5 is the X-ray flux between 0.1 and $2.4 \mathrm{keV}$ from the RASS survey (Voges et al. 1999, 2000). Parameters in columns $6-9$ of Table 8 have been omitted here since they are all blank for this sample.

(This table is available in machine-readable form.)

although the distribution for the blazars undetected by the LAT extends to significantly higher redshifts. Note that in contrast to $\mathrm{TeV}$ sources, the detection of high- $z$ sources in the LAT energy range is not strongly affected by gamma-gamma attenuation from the EBL. The highest-redshift BZCAT sources (56 have $z>3.1$ reaching $z=5.47$ ) are still eluding detection by the LAT. Figure 23 compares the distributions of radio flux at $1.4 \mathrm{GHz}$, optical $R$-band magnitude, and X-ray $(0.1-2.4 \mathrm{keV})$ flux between the BZCAT LAT-detected and non-LAT detected blazars, as well as the fraction of 3LAC sources relative to the total for a given flux. The gamma-ray loud blazars are somewhat brighter on average in all bands, confirming previous findings (Ackermann et al. 2011c; Lister et al. 2011). K-S tests give probabilities of $2 \times 10^{-11}, 2 \times 10^{-22}$, and $4 \times 10^{-19}$ that the $3 \mathrm{LAC}$ and non-3LAC distributions are drawn from the same population for the radio, optical, and X-ray cases, respectively. The fraction of gamma-ray loud blazars steadily decreases with decreasing radio, optical, and X-ray fluxes but remains non-negligible at the faint ends of the distributions. Figure 24 displays these radio-flux distributions broken down according to optical class. It is worth noting that some radio-

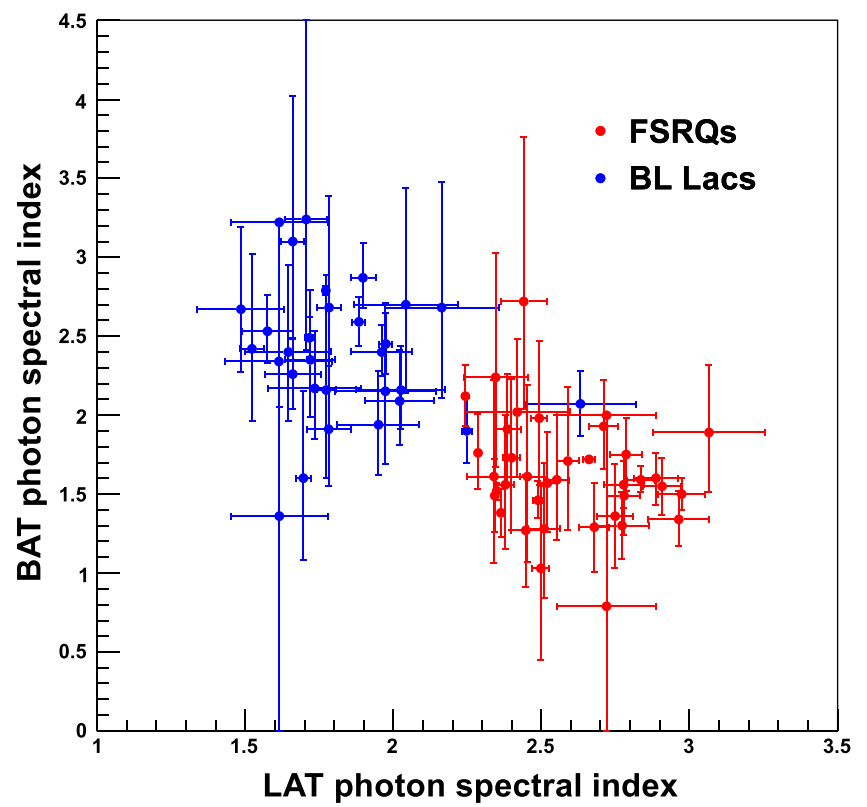

Figure 21. Photon spectral index in the BAT band (14-195 keV) vs. photon spectral index in the LAT band. Red: FSRQs; blue: BL Lacs.

bright blazars have not yet been detected by the LAT and that the detection fraction drops off with decreasing radio flux in a $\log$-linear fashion.

In Figure 25, the gamma-ray energy flux is plotted against the radio flux density at $1.4 \mathrm{GHz}$. A significant correlation is observed (Pearson correlation factor $=0.52$ ), confirming the findings in Ghirlanda et al. (2011) and Ackermann et al. (2011b). The best-fit power-law relation is $F_{\gamma} \simeq F_{r}^{0.34 \pm 0.05}$. Note that a stronger correlation is found if one uses the gammaray photon flux instead of the energy flux (Pearson correlation factor $=0.72)$, but this results from the photon-index dependence of the flux detection threshold in the gamma-ray band already discussed above. Radio-bright FSRQs have soft spectra in the LAT band and thus high detection thresholds, reinforcing the apparent correlation between radio flux density and gamma-ray fluxes.

The absence of a strong difference in the redshift or flux distributions between the detected and non-detected sets of blazars supports the conjecture that they belong to the same population of sources intermittently shining in gamma-rays. One can test the assumption that the fraction of non-detected sources is consistent with the variability properties assessed in Section 5.5 from the monthly light curves or if longer-term variability is required. Selecting BZCAT sources with high radio luminosity, $F_{\nu}>316 \mathrm{mJy}$, we obtain the gamma-ray energy flux distribution plotted in Figure 26. While 401 sources have been detected by the LAT, 706 sources with radio flux in the same range have not. Computing the dispersion of the 48 month flux average expected from the lognormal monthly flux distributions presented in Section 5.5 and using the centrallimit theorem, one obtains a typical value of $20 \%$ (illustrated by the blue arrows in Figure 26). This dispersion is obviously insufficient to account for the observed ratio between detected and non-detected blazars. Considerably longer timescales than those probed over the 4 year period (associated with physical or geometrical parameter(s) governing the observed jet gammaray/radio loudness ratio) must be in play. Since the fraction of 
Table 10

Properties of the 3FGL VHE AGNs

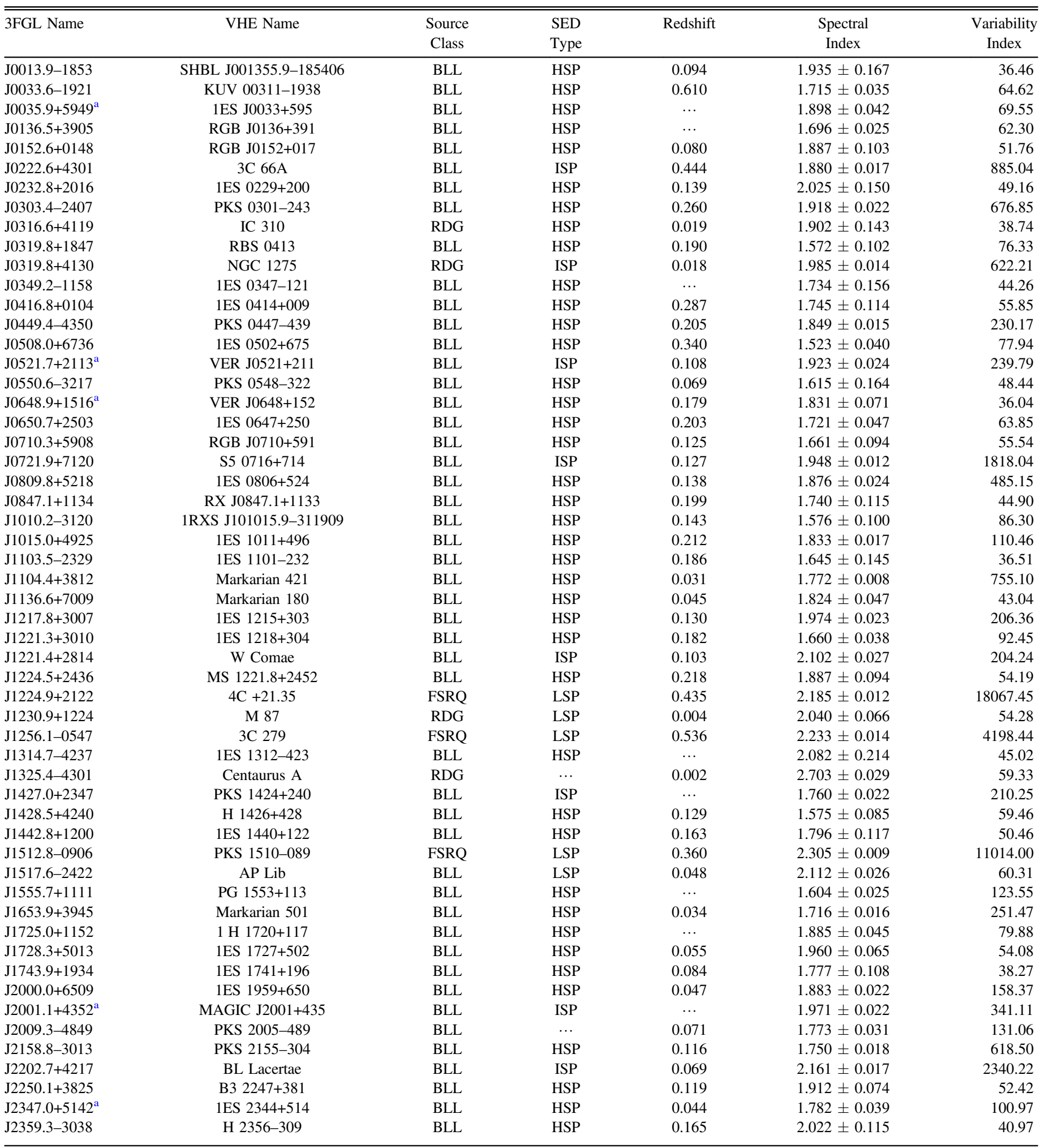

Note.

${ }^{a}$ Refers to low-latitude sources (not in 3LAC).

(This table is available in machine-readable form.)

LAT-detected FSRQs relative to the BZCAT total is less than that for BL Lacs (20\% versus $40 \%$ ), a larger amplitude variability of FSRQs is necessary to allow sources currently below the threshold to shine in gamma-rays at LAT-detection levels. This feature (a larger variability of FSRQs relative to BL Lacs) is compatible with the observations mentioned above. 


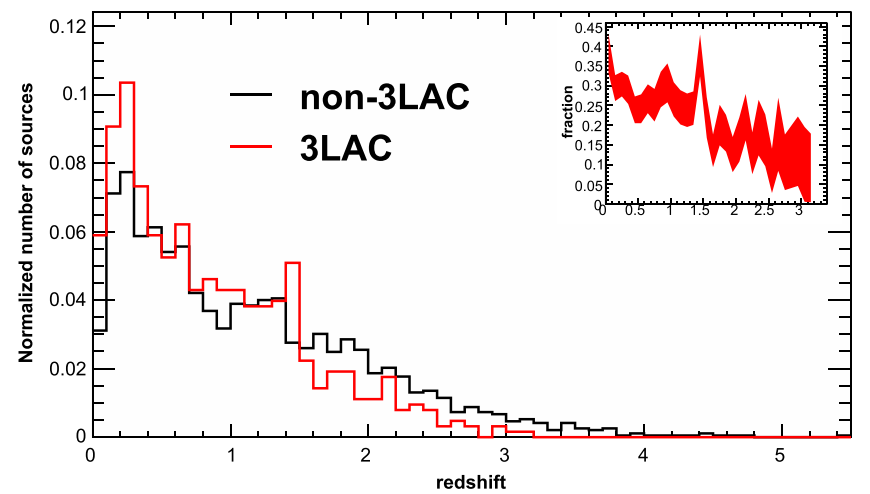

Figure 22. Redshift distributions for 3LAC (red) and non-3LAC (black) BZCAT sources. The inset shows the fraction of $3 \mathrm{LAC}$ sources relative to the total for a given redshift.
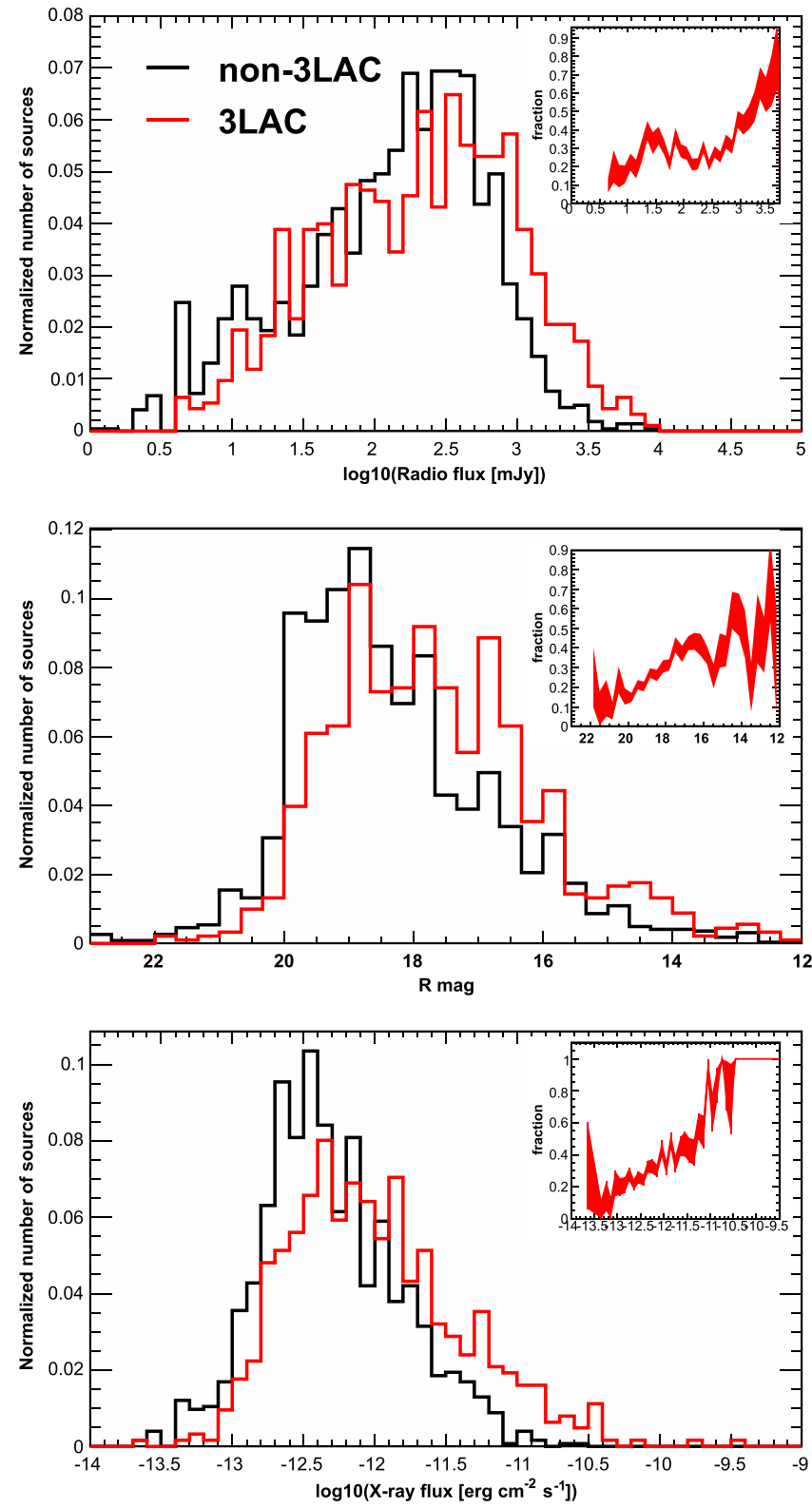

Figure 23. From top to bottom: radio flux density at $1.4 \mathrm{GHz}$, optical $R$ magnitude, X-ray flux $(0.1-2.4 \mathrm{keV})$ distributions for 3LAC (red), and non3LAC (black) BZCAT sources. The insets show the fraction of 3LAC sources relative to the total for a given flux.

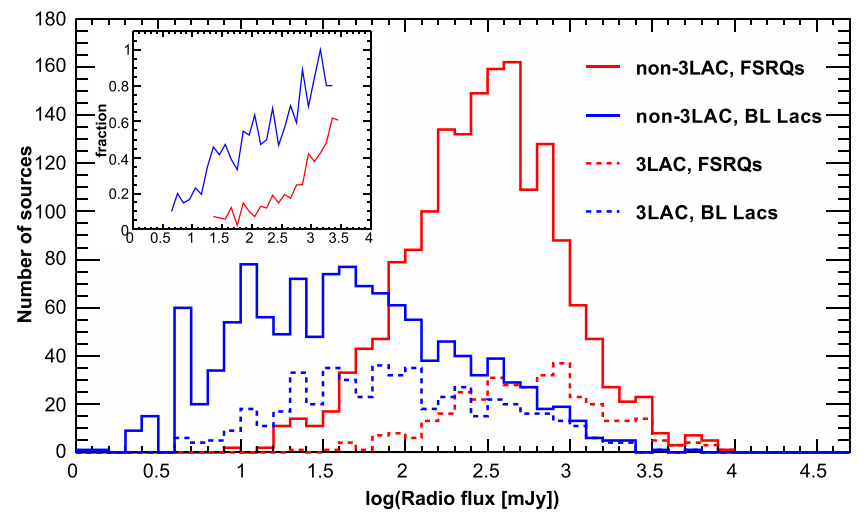

Figure 24. Radio flux density at $1.4 \mathrm{GHz}$ for $3 \mathrm{LAC}$ (dashed) and non-3LAC (solid) BZCAT sources. The inset displays the fraction of 3LAC sources relative to the total. Red: FSRQs; blue: BL Lacs.

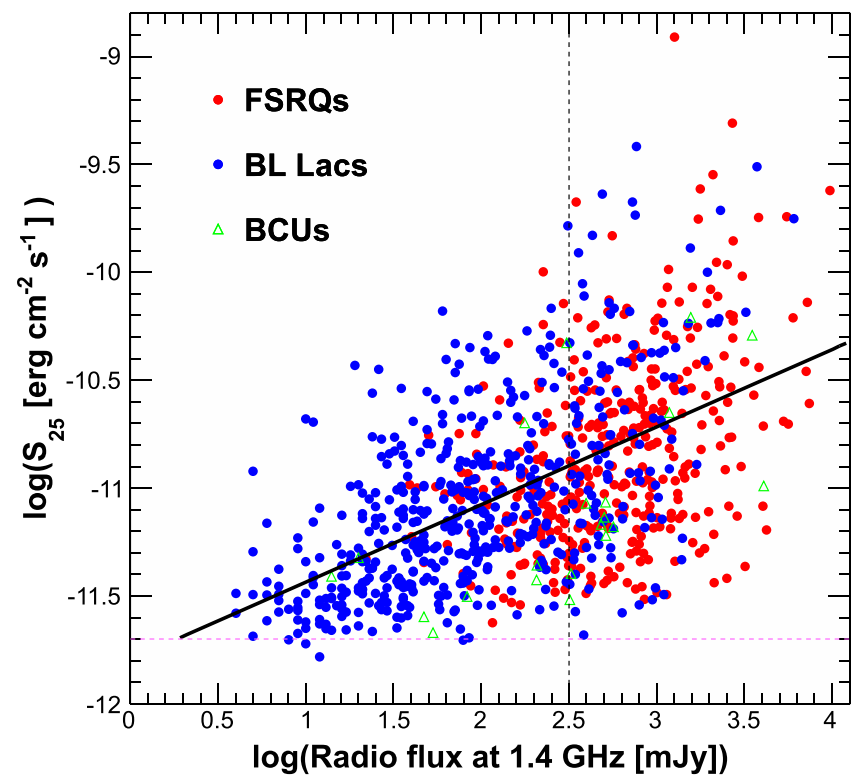

Figure 25. Gamma-ray energy flux plotted against the radio flux density at 1.4 GHz. Red circles: FSRQs; blue circles: BL Lacs; green triangles: BCUs. The horizontal dashed line depicts the approximate LAT detection limit and the vertical dashed line the lower limit of the selection used in Figure 26. The solid line depicts the result of the power-law fit described in the text.

\subsection{Compton Dominance}

We consider here the Compton dominance ratio $(\mathrm{CD})$, i.e., the ratio between the peak $\nu F_{\nu}$ for the high- and low-frequency SED humps, computed as described in Abdo et al. (2010a) and Finke (2013). The top panel of Figure 27 shows this ratio plotted against $\nu_{\text {peak }}^{\mathrm{S}}$ (similar to Figure 5 in Finke (2013), using 2LAC data). It is found that $\log C D$ has a mean and rms of $(0.60,0.65)$ for FSRQs and $(-0.11,0.48)$ for LSP-BL Lacs, while it has $(-0.39,0.42)$ for ISP-BL Lacs, and $(-0.78,0.39)$ for HSP-BL Lacs.

The spread in CD is partially driven by variability. The SED data are not simultaneous, especially for FSRQs, as some of them have displayed flux variations in gamma-rays greater than two orders of magnitude during the Fermi mission. However, as shown in Figure 26, the overall effect of variability on the mean gamma-ray flux is quite limited (see more below).

The combination of different beaming factors for the two humps (as expected if inverse-Compton off an external 


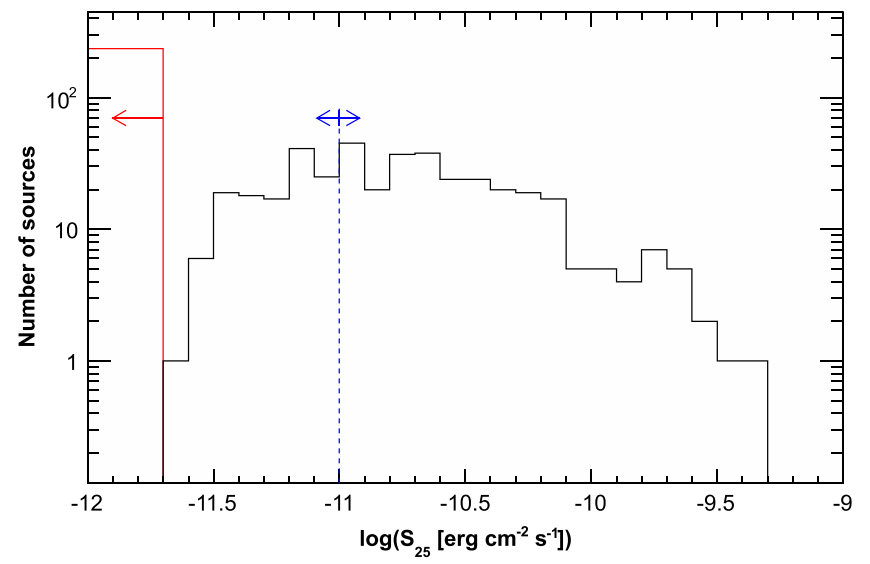

Figure 26. Distribution of gamma-ray energy flux for LAT-detected blazars with radio flux density at $1.4 \mathrm{GHz}$ above $316 \mathrm{mJy}$ (black). The arrows represent the $1 \sigma$ deviation expected for the 48 month average flux, assuming a log-normal energy-flux distribution with $\sigma_{\mathrm{Ln}}=1$ and a mean of $10^{-11} \mathrm{erg} \mathrm{cm}^{-2}$ $\mathrm{s}^{-\mathrm{T}}$. The red upper-limit histogram schematically represents the 706 non-LAT detected BZCAT blazars with radio fluxes in the same range.
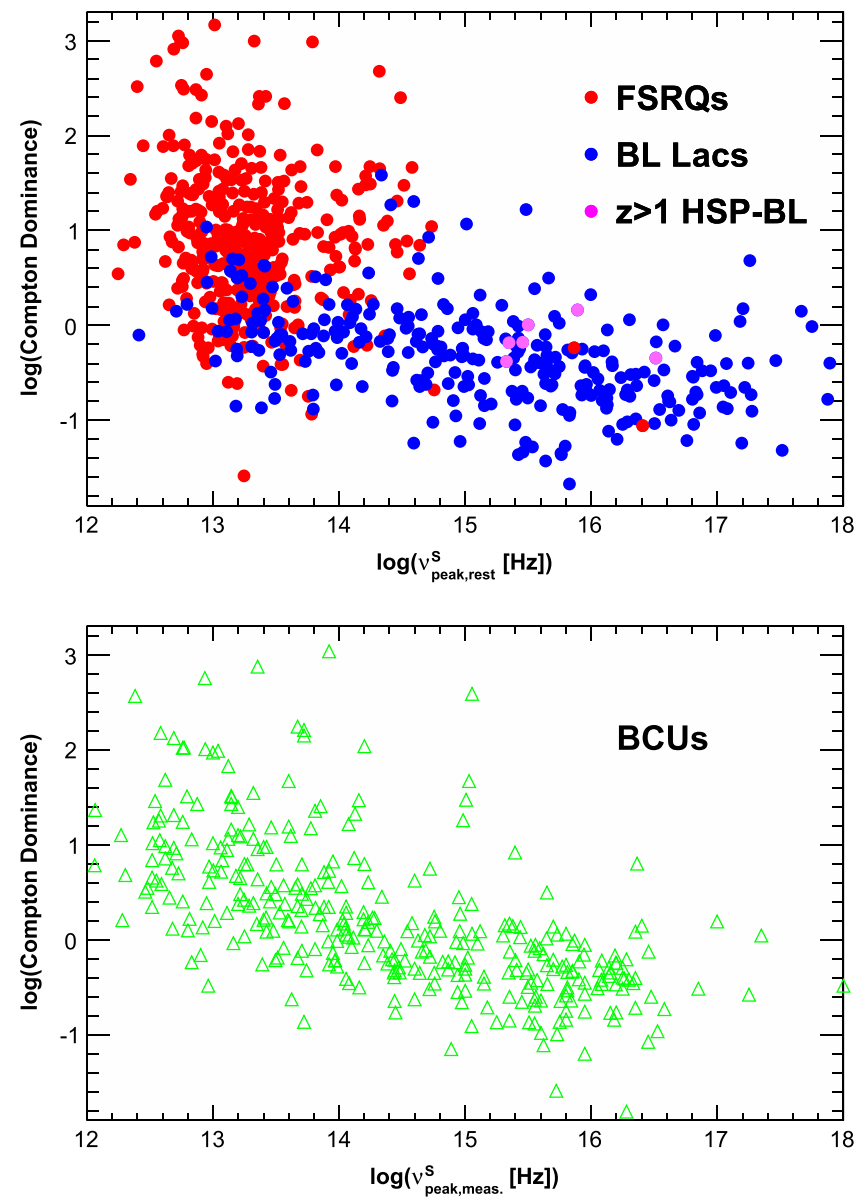

Figure 27. Top: Compton dominance vs. rest-frame peak synchrotron position. Red: FSRQs; blue: BL Lacs; magenta: z > 1 HSP-BL Lacs. Bottom: Compton dominance vs. observer-frame peak synchrotron position for BCUs.

radiation field is important, e.g., in FSRQs, Dermer 1995) and different jet angles relative to the line of sight within the $3 \mathrm{LAC}$ sample are likely to add to this spread. FSRQs have on average higher Compton dominance than BL Lacs, which exhibit a trend toward lower CD values with increasing $\nu_{\text {peak }}^{\mathrm{S}}$. Interestingly, as can be seen from Figure 27, the six luminous HSP-BL

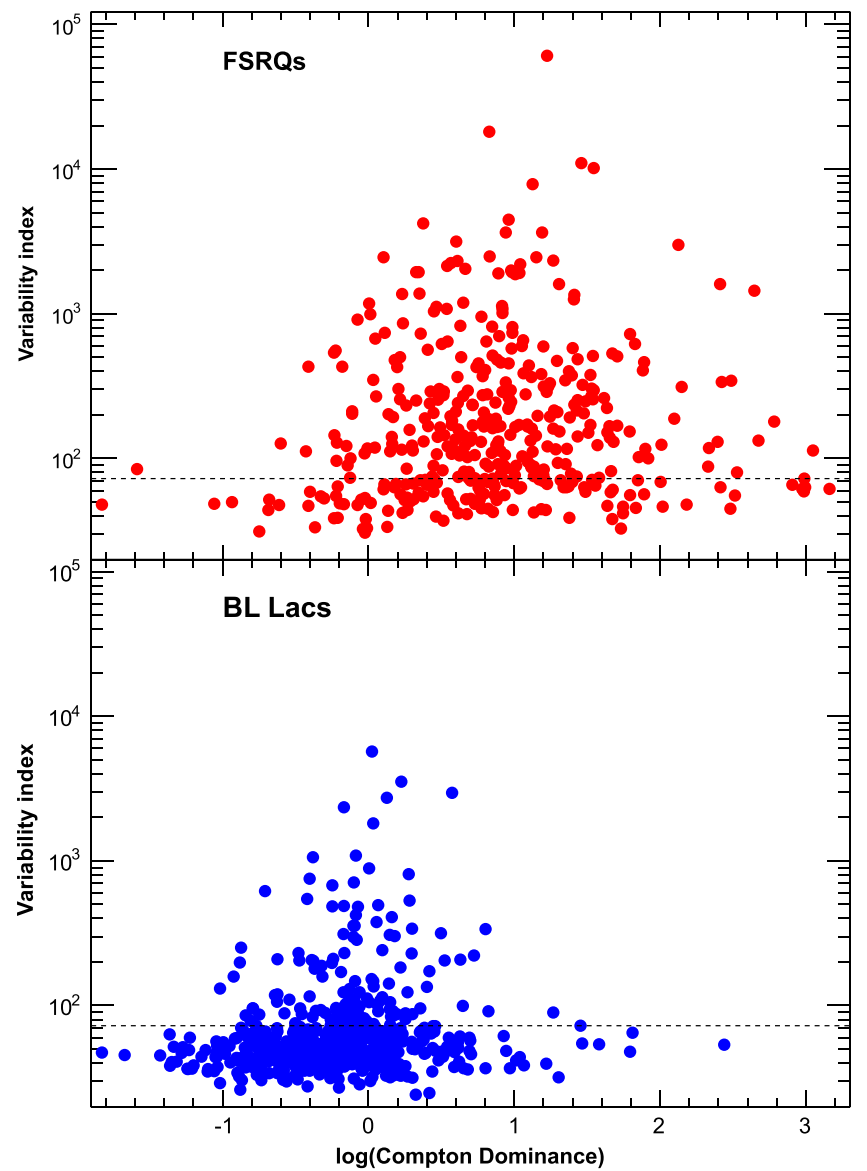

Figure 28. Variability index vs. Compton dominance. Top: FSRQs; bottom: BL Lacs. The dashed line corresponds to the $99 \%$ confidence level for a source to be variable.

Lacs located at redshifts greater than 1 show $C D$ values very similar to those located at low redshifts. These objects have a mean photon index of 1.94, comparable to the mean value of the whole HSP sample (1.88). Together, these features indicate that the overall SED shape of HSP-BL Lacs is not strongly dependent on redshift and thus neither on luminosity.

The lower panel of Figure 27 shows the corresponding plot for BCUs. Although $\nu_{\text {peak }}^{\mathrm{S}}$ has not been corrected by $(1+z)$ for most sources as their redshifts are unknown, the observed trend is very similar to that of blazars with known types.

An interesting point regards the comparison between LSPBL Lacs and FSRQs. The gamma-ray properties of the former being intermediate between those of FSRQs and of HSP-BL Lacs, they could be FSRQs "in disguise" where the emission lines are swamped by a strong non-thermal continuum as suggested by Giommi et al. (2013). Figure 28 shows the variability index plotted against CD for FSRQs and BL Lacs. It is seen that the regions occupied by the BL Lacs and FSRQs have moderate overlap.

\section{3. $\log N-\log S$}

Figure 29 shows the $\log N-\log S$ ( $S$ being the gamma-ray energy flux and $N$ the cumulative number of sources above this flux) plot for the full 1LAC, 2LAC, and 3LAC catalogs, as well as for FSRQs, BL Lacs, and BCUs in the respective Clean Samples, uncorrected for coverage. Note that the LAT limiting 


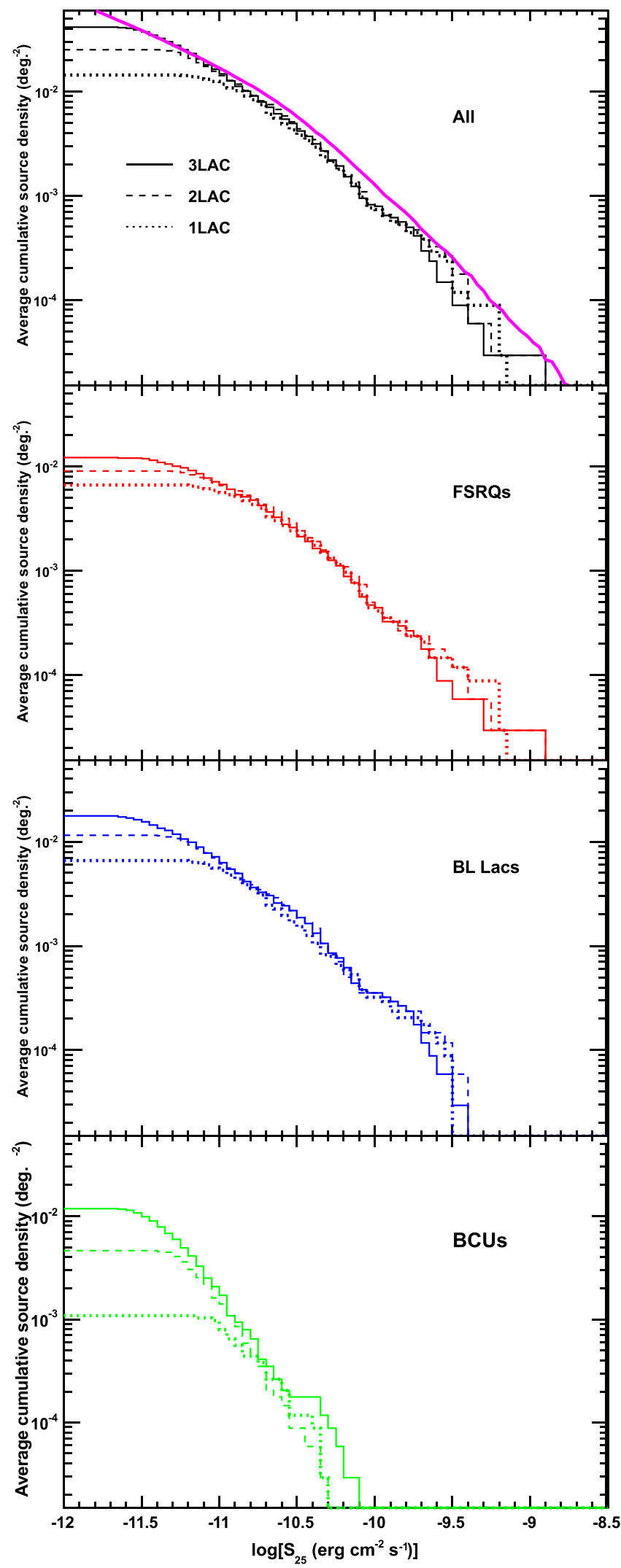

Figure 29. Cumulative energy flux distributions (uncorrected for non-uniform sensitivity and detection/association efficiency) for blazars in Clean Samples. Solid: 3LAC; dashed: 2LAC; dotted: 1LAC. Top: Total. The magenta curve corresponds to the predictions derived from Ackermann et al. (2012d). Second: FSRQs. Third: BL Lacs. Bottom: blazars of unknown type. energy flux is essentially independent of the photon index and thus of the blazar class as illustrated in Figure 9. A steady increase in the number of sources is observed for all classes, with the 3LAC being roughly in line with extrapolations from the 2LAC. Power-law fits performed on the 3LAC distributions between somewhat arbitrary energy-flux limits (see Figure 29) yield slopes of 1.23, 1.22, and 1.09 for the whole set, FSRQs, and BL Lacs, respectively. Integrating the energy-flux distributions above $100 \mathrm{MeV}$ in the range $10^{-11}$ $10^{-9} \mathrm{erg} \mathrm{cm}^{-2} \mathrm{~s}^{-1}$ gives gamma-ray intensities for all sources and FSRQs of $1.4 \times 10^{-6} \mathrm{GeV} \mathrm{cm}^{-2} \mathrm{~s}^{-1} \mathrm{sr}^{-1}$ and $4.7 \times 10^{-7}$ $\mathrm{GeV} \mathrm{cm} \mathrm{s}^{-1} \mathrm{sr}^{-1}$, respectively. These results can be compared to those obtained in assessing the diffuse gamma-ray emission (Ackermann et al. 2012d) : the intensity for all resolved sources at $|b|>20^{\circ}$ is estimated to be $9.5 \times 10^{-7} \mathrm{GeV} \mathrm{cm}^{-2} \mathrm{~s}^{-1} \mathrm{sr}^{-1}$. This corresponds to $1.2 \times 10^{-6} \mathrm{GeV} \mathrm{cm}^{-2} \mathrm{~s}^{-1} \mathrm{sr}^{-1}$ after applying the geometrical correction (from $|b|>20^{\circ}$ to $|b|>10^{\circ}$ ), in reasonable agreement with the 3 LAC-based estimate.

\section{CONCLUSIONS}

We have presented the third catalog of LAT-detected AGNs (3LAC), based on 48 months of LAT data. This is an improvement over the 1LAC (11 months of data) and 2LAC (24 months of data) in terms of data quality and analysis methods. Key results from the 3LAC sample include the following.

1. An increase of $71 \%$ in the number of blazars relative to 2LAC stems from the two-fold increase in exposure and the use of improved counterpart catalogs. The energy-flux distributions of the different blazar populations are in good agreement with extrapolation from earlier catalogs.

2. A significant increase of the non-blazar population is found with respect to previous catalogs. The new sources include: two FRIIs (Pictor A, 3C 303), three FRIs $(4 \mathrm{C}+39.12$, 3C $189,3 \mathrm{C} 264$ plus one possible association, Fornax A), and four SSRQs (TXS 0826+091, $4 \mathrm{C}+0.40,3 \mathrm{C} 275.1,3 \mathrm{C} 286)$. However, other sources (3C 407, NGC 6951, NGC 6814) reported in previous catalogs are now missing.

3. A large fraction $(>75 \%)$ of Swift hard X-ray BATdetected blazars and all but one TeV-detected AGNs have now been detected by the Fermi-LAT.

4. The most distant 3LAC blazar is the same as in 1LAC and 2LAC: PKS 0537-286, lying at $z=3.1$. Many BZCAT blazars at higher redshifts have yet to be detected by the LAT. Although $50 \%$ of the BL Lacs still do not have measured redshifts, upper limits have recently been obtained for 134 2LAC sources and lower limits as well for 57 of them. These constraints indicate that the measured redshifts are biased low for BL Lacs. Using the luminosities derived from these constraints, the sources populate a previously scarcely occupied area in the $L_{\gamma}-\Gamma$ diagram, somewhat undermining the picture of the blazar sequence.

5. Along the same lines, a few rare outliers (four highluminosity HSP BLLacs and two HSP FSRQs) are included in the 3LAC, while they were missing in 2LAC. 
The high-luminosity HSP-BL Lacs exhibit Compton dominance values similar to the bulk of that class.

6. The main properties of blazars previously reported in 1LAC and 2LAC are confirmed. The average photon index, gamma-ray luminosity, flux variability, and spectral curvature monotonically evolve from FSRQs to HSP BL Lacs, with LSP- and ISP-BL Lacs showing intermediate behavior.

7. The fraction of 3LAC blazars in the total population of blazars listed in BZCAT remains non-negligible even at the faint ends of the BZCAT-blazar radio, optical, and $\mathrm{X}$-ray flux distributions, which is a clue that even the faintest, and thus possibly all, known blazars could eventually shine in gamma-rays at LAT-detection levels. A larger fraction $(44 \%)$ of the known BL Lacs than FSRQs $(24 \%)$ has been detected so far. The duty cycle of FSRQs appears to be longer than four years if most of them are eventual gamma-ray emitters.

The 3LAC catalog is intended to serve as a valuable resource for a better understanding of the gamma-ray loud AGNs. The next LAT AGN catalog will benefit from the improved Pass 8 data selection and IRFs (Atwood et al. 2013). Pass 8 is the result of a comprehensive revision of the entire event-level analysis, based on the experience gained in the prime phase of the mission. The gain in effective area at the low end of the LAT energy range will be particularly notable. The 4LAC catalog is thus expected to include a non-incremental number of new, especially soft-spectrum AGNs.

The Fermi LAT Collaboration acknowledges generous ongoing support from a number of agencies and institutes that have supported both the development and the operation of the LAT as well as scientific data analysis. These include the National Aeronautics and Space Administration and the Department of Energy in the United States, the Commissariat à l'Energie Atomique and the Centre National de la Recherche Scientifique/Institut National de Physique Nucléaire et de Physique des Particules in France, the Agenzia Spaziale Italiana and the Istituto Nazionale di Fisica Nucleare in Italy, the Ministry of Education, Culture, Sports, Science and Technology (MEXT), High Energy Accelerator Research Organization (KEK) and Japan Aerospace Exploration Agency (JAXA) in Japan, and the K. A. Wallenberg Foundation, the Swedish Research Council, and the Swedish National Space Board in Sweden. Additional support for science analysis during the operations phase is gratefully acknowledged from the Istituto Nazionale di Astrofisica in Italy and the Centre National d'Études Spatiales in France.

This research has made use of data obtained from the highenergy Astrophysics Science Archive Research Center (HEASARC) provided by NASA's Goddard Space Flight Center; the SIMBAD database operated at CDS, Strasbourg, France; and the NASA/IPAC Extragalactic Database (NED) operated by the Jet Propulsion Laboratory, California Institute of Technology, under contract with the National Aeronautics and Space Administration. This research has made use of data archives, catalogs, and software tools from the ASDC, a facility managed by the Italian Space Agency (ASI). Part of this work is based on the NVSS. The National Radio Astronomy Observatory is operated by Associated Universities, Inc., under contract with the National Science Foundation. This publication makes use of data products from the Two Micron All Sky Survey, which is a joint project of the University of Massachusetts and the Infrared Processing and Analysis Center/California Institute of Technology, funded by the National Aeronautics and Space Administration and the National Science Foundation. This publication makes use of data products from the Wide-field Infrared Survey Explorer, which is a joint project of the University of California, Los Angeles, and the Jet Propulsion Laboratory/California Institute of Technology, funded by the National Aeronautics and Space Administration. Funding for the SDSS and SDSS-II has been provided by the Alfred P. Sloan Foundation, the Participating Institutions, the National Science Foundation, the U.S. Department of Energy, the National Aeronautics and Space Administration, the Japanese Monbukagakusho, the Max Planck Society, and the Higher Education Funding Council for England. The SDSS Web Site is http://www.sdss.org/. The SDSS is managed by the Astrophysical Research Consortium for the Participating Institutions. The Participating Institutions are the American Museum of Natural History, Astrophysical Institute Potsdam, University of Basel, University of Cambridge, Case Western Reserve University, University of Chicago, Drexel University, Fermilab, the Institute for Advanced Study, the Japan Participation Group, Johns Hopkins University, the Joint Institute for Nuclear Astrophysics, the Kavli Institute for Particle Astrophysics and Cosmology, the Korean Scientist Group, the Chinese Academy of Sciences (LAMOST), Los Alamos National Laboratory, the Max-Planck-Institute for Astronomy (MPIA), the Max-Planck-Institute for Astrophysics (MPA), New Mexico State University, Ohio State University, University of Pittsburgh, University of Portsmouth, Princeton University, the United States Naval Observatory, and the University of Washington.

Facilities: Fermi LAT.

\section{APPENDIX \\ NOTE ON CONVENTION FOR SOURCE ASSOCIATION COUNTERPART NOMENCLATURE}

In this paper we have tentatively adopted a history-based rationale for the names of blazar and other AGN source counterparts associated with 3LAC sources, as reported in the 3FGL catalog FITS file. ${ }^{89}$ This naming rationale is already working as the source name resolver in NED (NASA/IPAC Extragalactic Database), and was already in use, in part, in the 2LAC paper. It is possible to retrieve an approximate knowledge about the chronological appearance of a radio/ optical/X-ray point source in past catalogs thanks to NED, Simbad-Vizier, and ADS databases. The best-known (widely used) naming rationale is more arbitrary and more difficult to reconstruct, it suffers more from subjectivity, and applies only to a minority of the brightest blazars/AGNs.

AGNs and blazars were first discovered as optical non-starlike/nebula objects (i.e., galaxies, M, NGC, IC catalogs published between 1781 and 1905), as optical variable stars (Argelander designations for BL Lac, W Com, AP Lib), unusually optically blue starlike objects (Ton, PHL, Mkn catalogs all published between about 1957 and 1974), and subsequent catalogs of normal or peculiar galaxies (CGCG, MCG, CGPG, UGC, Ark, Zw/I-V, Tol catalogs all published between about 1961 and 1976). Subsequent optical catalogs like the PG, PB, US, SBS, PGC, LEDA, HS, and SDSS are

$\overline{89}$ http://fermi.gsfc.nasa.gov/ssc/data/access/lat/4yr_catalog/gll_psc_v16.fit 
also used in our 3LAC associations naming rationale. ${ }^{90}$ In parallel, most blazars and AGNs were detected as new discrete point sources in the first radio observations and surveys (sources like Vir A, Cen A, Cen B, Per A, etc., in the early 1950s, then the 3C, CTA, PKS, 4C, O[+letter], VRO, NRAO, AO, DA, B2, GC, S1/S2/S3 catalogs all published between about 1959 and 1974). Other subsequent radio catalogs like the TXS, 5C, S4/S5, MRC, B3 (all about 1974-1985) and MG1/ MG2/MG4, 87 GB, 6C/7C, JVAS, PMN, EF, CJ2, FIRST, Cul, GB6, FBQS, WN, NVSS, CLASS, IERS, SUMSS, CRATES (all after 1986) are also used in our work. Other catalogs of interest at IR or UV frequencies for purposes of 3LAC association names are the KUV, EUVE, 2MASSi, and 2MASS. Additional blazars that are fainter in the radio/optical bands were discovered directly thanks to the first X-ray observations (2A, 4U, XRS, EXO, H/1 H, MS, 1E, 1ES, 2E, and RX all published from about 1978 to the mid 1990s). The subsequent (after 1997) reanalysis and catalog constructions based mainly on the ROSAT survey and radio-X-ray source cross correlations are also used in the 3LAC (RGB, RBS, RHS, 1RXS, XSS catalogs).

The most common source counterpart roots in 3LAC associations have origins in the $3 \mathrm{C}, 4 \mathrm{C}, \mathrm{PKS}, \mathrm{O}[+$ letter], $\mathrm{B} 2$, S2/S3/S5, TXS, MG1/MG2, PMN, GB6, SDSS, 1ES, RX, RBS, and 1RXS catalogs. PKS (Parkes Radio Catalog, Australia) chronologically is the source name preferred for southern celestial radio sources, over almost all the other epoch-overlapping radio catalogs. The survey for northern celestial radio sources at Parkes likely started after the more easily observable southern sources, therefore later than the $\mathrm{O}$ [+letter] (Ohio State University Radio Survey Catalog, USA) observations, and certainly after the $3 \mathrm{C}$ and $4 \mathrm{C}$ catalogs. The procedure for selecting source counterpart names is tuned to the most-used/known criterion for the most famous sources (for example OJ 287 instead of PKS 0851+202/ PG 0851+202, but PKS 0735+17 instead of OI 158 / DA 237). Other famous blazars/AGN sources are more likely to follow the best-known criterion (example: Cen A is more frequently used than NGC 5128 , even though this galaxy was first discovered in the NGC catalog). For the northern celestial hemisphere the preferred radio source name chosen following the approximate chronological criterion follows the sequence of radio catalogs reported above $(3 \mathrm{C}, \mathrm{CTA}, 4 \mathrm{C}$, O[+letter], NRAO, AO, DA, B2, GC, S1/S2/S3, TXS, MG1/MG2/MG4, etc.). Some catalog designations (like the $87 \mathrm{~GB}$ and rare optical names) are essentially not used in the 3LAC. RBG names have been preferred to RBS and 1RXS names, and the NVSS names have been preferred to the SDSS names. We do not have a preference between GB6 and RX names or between RBS and 1RXS names, as all are being used arbitrarily.

\section{REFERENCES}

Abdo, A. A., Ackermann, M., Agudo, I., et al. 2010a, ApJ, 716, 30 Abdo, A. A., Ackermann, M., Ajello, M., et al. 2009a, ApJ, 700, 597 Abdo, A. A., Ackermann, M., Ajello, M., et al. 2009b, ApJ, 699, 817 Abdo, A. A., Ackermann, M., Ajello, M., et al. 2009c, ApJ, 699, 31 Abdo, A. A., Ackermann, M., Ajello, M., et al. 2009d, ApJ, 707, 55 Abdo, A. A., Ackermann, M., Ajello, M., et al. 2009e, ApJS, 183, 46 Abdo, A. A., Ackermann, M., Ajello, M., et al. 2010b, Sci, 328, 725

\footnotetext{
${ }^{90}$ For all catalogs cited in this appendix, the pertaining literature and bibliographic references can be directly retrieved through the NED web database at ned.ipac.caltech.edu/cgi-bin/catdef?prefix $=X Y Z$, where "XYZ" is the catalog/list code or prefix (e.g., "B2").
}

Abdo, A. A., Ackermann, M., Ajello, M., et al. 2010c, ApJ, 723, 1082 Abdo, A. A., Ackermann, M., Ajello, M., et al. 2010d, ApJS, 188, 405 Abdo, A. A., Ackermann, M., Ajello, M., et al. 2010e, ApJ, 720, 912 Abdo, A. A., Ackermann, M., Ajello, M., et al. 2010f, ApJ, 710, 1271 Abdo, A. A., Ackermann, M., Ajello, M., et al. 2010g, ApJ, 715, 429 Abdo, A. A., Ackermann, M., Ajello, M., et al. 2011, ApJL, 733, L26+ Abramowski, A., Acero, F., Akhperjanian, A. G., et al. 2013, A\&A, 552, A118 Ackermann, M., Ajello, M., Allafort, A., et al. 2011a, ApJ, 741, 30 Ackermann, M., Ajello, M., Allafort, A., et al. 2011b, ApJ, 741, 30 Ackermann, M., Ajello, M., Allafort, A., et al. 2011c, ApJ, 743, 171 Ackermann, M., Ajello, M., Allafort, A., et al. 2012a, ApJ, 755, 164 Ackermann, M., Ajello, M., Allafort, A., et al. 2012b, ApJ, 747, 104 Ackermann, M., Ajello, M., Allafort, A., et al. 2012c, Sci, 338, 1190 Ackermann, M., Ajello, M., Allafort, A., et al. 2013, ApJS, 209, 34 Ackermann, M., Ajello, M., Allafort, A., et al. 2015, ApJ, 806, 1 Ackermann, M., Ajello, M., Atwood, W. B., et al. 2012d, ApJ, 750, 3 Ackermann, M., Ajello, M., Baldini, L., et al. 2010, ApJ, 721, 1383 Ahn, C. P., Alexandroff, R., Allende Prieto, C., et al. 2012, ApJS, 203, 21 Ajello, M., Romani, R. W., Gasparrini, D., et al. 2014, ApJ, 780, 73 Ajello, M., Shaw, M. S., Romani, R. W., et al. 2012, ApJ, 751, 108 Aleksić, J., Ansoldi, S., Antonelli, L. A., et al. 2014, Sci, 346, 1080 Arsioli, B., Fraga, B., Giommi, P., Padovani, P., \& Marrese, M. 2015, arXiv: 1504.02801

Atwood, W., Albert, A., Baldini, L., et al. 2013, arXiv:1303.3514

Atwood, W. B., Abdo, A. A., Ackermann, M., et al. 2009, ApJ, 697, 1071

Baumgartner, W. H., Tueller, J., Markwardt, C. B., et al. 2013, ApJS, 207, 19 Böttcher, M. 2007, Ap\&SS, 309, 95

Brown, A. M., \& Adams, J. 2012, MNRAS, 421, 2303

Burgess, A. M., \& Hunstead, R. W. 2006, AJ, 131, 114

Cerruti, M., Dermer, C. D., Lott, B., Boisson, C., \& Zech, A. 2013, ApJL, 771, L4

Cheung, C. C. 2007, in ASP Conf. Ser. 373, The Central Engine of Active Galactic Nuclei, ed. L. C. Ho \& J.-W. Wang (San Francisco, CA: ASP), 255

Chiang, J. 2012, in Advances in Machine Learning and Data Mining for Astronomy, ed. M. J. Way et al. (London: Taylor \& Francis)

Ciprini, S., \& Thompson, D. J. 2013, in IV Fermi Symp. Proc., eConf C121028 17, arXiv: 1303.4054

Condon, J. J., Cotton, W. D., Greisen, E. W., et al. 1998, AJ, 115, 1693

D’Abrusco, R., Massaro, F., Ajello, M., et al. 2012, ApJ, 748, 68

D’Abrusco, R., Massaro, F., Paggi, A., et al. 2013, ApJS, 206, 12

D'Ammando, F., Orienti, M., Finke, J., et al. 2012, MNRAS, 426, 317

D’Ammando, F., Orienti, M., Finke, J., et al. 2013, MNRAS, 436, 191

de Ruiter, H. R., Arp, H. C., \& Willis, A. G. 1977, A\&AS, 28, 211

Dermer, C. D. 1995, ApJL, 446, L63

Donato, D., \& Perkins, J. 2011, ATel, 3452, 1

Fanaroff, B. L., \& Riley, J. M. 1974, MNRAS, 167, 31P

Fermi-LAT Collaboration 2015, arXiv:1501.02003

Finke, J. D. 2013, ApJ, 763, 134

Finke, J. D., \& Dermer, C. D. 2010, ApJL, 714, L303

Fomalont, E. B., Ebneter, K. A., van Breugel, W. J. M., \& Ekers, R. D. 1989, ApJ, 346, 17

Georganopoulos, M., Sambruna, R. M., Kazanas, D., et al. 2008, ApJL, 686, L5 Ghirlanda, G., Ghisellini, G., Tavecchio, F., Foschini, L., \& Bonnoli, G. 2011, MNRAS, 413, 852

Ghisellini, G., Haardt, F., della Ceca, R., Volonteri, M., \& Sbarrato, T. 2013, MNRAS, 432, 2818

Ghisellini, G., Maraschi, L., \& Tavecchio, F. 2009, MNRAS, 396, L105

Ghisellini, G., Tavecchio, F., Foschini, L., et al. 2012, MNRAS, 425, 1371

Giebels, B., \& Degrange, B. 2009, A\&A, 503, 797

Giommi, P., Padovani, P., \& Polenta, G. 2013, MNRAS, 431, 1914

Giommi, P., Polenta, G., Lähteenmäki, A., et al. 2012, A\&A, 541, A160

Giovannini, G., Cotton, W. D., Feretti, L., Lara, L., \& Venturi, T. 2001, ApJ, 552,508

Grandi, P., Torresi, E., \& Stanghellini, C. 2012, ApJL, 751, L3

Hartman, R. C., Bertsch, D. L., Bloom, S. D., et al. 1999, ApJS, 123, 79

Hartman, R. C., Kadler, M., \& Tueller, J. 2008, ApJ, 688, 852

Hayashida, M., Stawarz, Ł., Cheung, C. C., et al. 2013, ApJ, 779, 131

Healey, S. E., Romani, R. W., Taylor, G. B., et al. 2007, ApJS, 171, 61

Hough, D. H. 2013, European Physical Journal Web of Conferences, 61, 08009

Jones, D. H., Read, M. A., Saunders, W., et al. 2009, MNRAS, 399, 683

Kadler, M., Eisenacher, D., Ros, E., et al. 2012a, A\&A, 538, L1

Kataoka, J., Stawarz, Ł., Cheung, C. C., et al. 2010, ApJ, 715, 554

Kataoka, J., Stawarz, L., Takahashi, Y., et al. 2011, ApJ, 740, 29

Katsuta, J., Tanaka, Y. T., Stawarz, L., et al. 2013, A\&A, 550, A66

Kovalev, Y. Y., Aller, H. D., Aller, M. F., et al. 2009, ApJL, 696, L17 
Kovalev, Y. Y., Petrov, L., Fomalont, E. B., \& Gordon, D. 2007, AJ, 133, 1236 Laing, R. A., Riley, J. M., \& Longair, M. S. 1983, MNRAS, 204, 151 Landt, H. 2012, MNRAS, 423, L84

Lenain, J.-P., Ricci, C., Türler, M., Dorner, D., \& Walter, R. 2010, A\&A, 524, A72

Lister, M. L., Aller, M., Aller, H., et al. 2011, ApJ, 742, 27

Lister, M. L., Aller, M. F., Aller, H. D., et al. 2013, AJ, 146, 120

Lister, M. L., Homan, D. C., Kadler, M., et al. 2009, ApJL, 696, L22

Lonsdale, C., Conrow, T., Evans, T., et al. 1998, in IAU Symp. 179, New Horizons from Multi-Wavelength Sky Surveys, ed. B. J. McLean et al. (Dordrecht: Kluwer Academic Publishers), 450-+

Masci, F. J., Condon, J. J., Barlow, T. A., et al. 2001, PASP, 113, 10

Massaro, E., Giommi, P., Leto, C., et al. 2009, A\&A, 495, 691

Massaro, E., Perri, M., Giommi, P., \& Nesci, R. 2004, A\&A, 413, 489

Massaro, F., D'Abrusco, R., Tosti, G., et al. 2012, ApJ, 750, 138

Mauch, T., Murphy, T., Buttery, H. J., et al. 2003, MNRAS, 342, 1117

McConville, W., Ostorero, L., Moderski, R., et al. 2011, ApJ, 738, 148

Meyer, E. T., Fossati, G., Georganopoulos, M., \& Lister, M. L. 2012, ApJL, 752, L4

Mukherjee, R., Halpern, J., Mirabal, N., \& Gotthelf, E. V. 2002, ApJ, 574, 693 Murphy, T., Sadler, E. M., Ekers, R. D., et al. 2010, MNRAS, 402, 2403

Myers, S. T., Jackson, N. J., Browne, I. W. A., et al. 2003, MNRAS, 341, 1

Neronov, A., Semikoz, D., \& Vovk, I. 2010, A\&A, 519, L6

Nolan, P. L., Abdo, A. A., Ackermann, M., et al. 2012, ApJS, 199, 31

Ojha, R., Kadler, M., Böck, M., et al. 2010, A\&A, 519, A45

Pacciani, L., Tavecchio, F., Donnarumma, I., et al. 2014, ApJ, 790, 45

Padovani, P., Giommi, P., \& Rau, A. 2012, MNRAS, 422, L48

Planck Collaboration, Ade, P. A. R., Aghanim, N., et al. 2011, A\&A, 536, A7
Planck Collaboration, Ade, P. A. R., Aghanim, N., et al. 2014, A\&A, 571, A16

Peter, D., Domainko, W., Sanchez, D. A., van der Wel, A., \& Gässler, W. 2014, A\&A, 571, A41

Petrov, L., Mahony, E. K., \& Edwards, P. G. 2013, MNRAS, 432, 1294

Piner, B. G., Pushkarev, A. B., Kovalev, Y. Y., et al. 2012, ApJ, 758, 84

Poutanen, J., \& Stern, B. 2010, ApJL, 717, L118

Prestage, R. M., \& Peacock, J. A. 1983, MNRAS, 204, 355

Ruan, J. J., Anderson, S. F., Plotkin, R. M., et al. 2014, ApJ, 797, 19

Shaw, M. S., Romani, R. W., Cotter, G., et al. 2013, ApJ, 764, 135

Sokolovsky, K. V., Schinzel, F. K., Tanaka, Y. T., et al. 2014, A\&A, 565,26

Sreekumar, P., Bertsch, D. L., Hartman, R. C., Nolan, P. L., \& Thompson, D. J. 1999, APh, 11, 221

Strong, A. W., \& Bignami, G. F. 1983, ApJ, 274, 549

Sutherland, W., \& Saunders, W. 1992, MNRAS, 259, 413

Takeuchi, Y., Kataoka, J., Stawarz, Ł., et al. 2012, ApJ, 749, 66

Tanaka, Y. T., Cutini, S., Ciprini, S., et al. 2014, ATel, 6529, 1

Tavecchio, F., Pacciani, L., Donnarumma, I., et al. 2013, MNRAS, 435, L24

Teng, S. H., Mushotzky, R. F., Sambruna, R. M., Davis, D. S., \& Reynolds, C. S. 2011, ApJ, 742, 66

Tluczykont, M., Bernardini, E., Satalecka, K., et al. 2010, A\&A, 524, A48

Véron-Cetty, M.-P., \& Véron, P. 2010, A\&A, 518, A10+

Voges, W., Aschenbach, B., Boller, T., et al. 1999, A\&A, 349, 389

Voges, W., Aschenbach, B., Boller, T., et al. 2000, yCat, 9029, 0

Wilkinson, P. N., Akujor, C. E., Cornwell, T. J., \& Saikia, D. J. 1991, MNRAS, 248, 86

Wright, A. E., Griffith, M. R., Hunt, A. J., et al. 1996, ApJS, 103, 145 